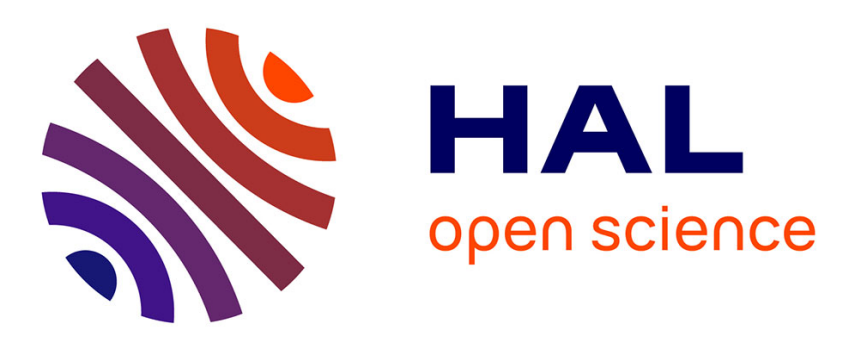

\title{
Zigzag instability of vortex pairs in stratified and rotating fluids. Part 1 . General stability equations. Paul Billant
}

\section{To cite this version:}

Paul Billant. Zigzag instability of vortex pairs in stratified and rotating fluids. Part 1. General stability equations.. Journal of Fluid Mechanics, 2010, 660 (october), pp.354-395. 10.1017/s0022112010002818. hal-01021122

HAL Id: hal-01021122

https://hal-polytechnique.archives-ouvertes.fr/hal-01021122

Submitted on 16 Jul 2014

HAL is a multi-disciplinary open access archive for the deposit and dissemination of scientific research documents, whether they are published or not. The documents may come from teaching and research institutions in France or abroad, or from public or private research centers.
L'archive ouverte pluridisciplinaire HAL, est destinée au dépôt et à la diffusion de documents scientifiques de niveau recherche, publiés ou non, émanant des établissements d'enseignement et de recherche français ou étrangers, des laboratoires publics ou privés. 


\title{
Zigzag instability of vortex pairs in stratified and rotating fluids. Part 1 . General stability equations.
}

\author{
PAUL BILLANT† \\ LadHyX, CNRS, École Polytechnique, F-91128 Palaiseau Cedex, France \\ (Received 5 May 2009; revised 12 May 2010; accepted 13 May 2010; \\ first published online 21 July 2010)
}

In stratified and rotating fluids, pairs of columnar vertical vortices are subjected to three-dimensional bending instabilities known as the zigzag instability or as the tall-column instability in the quasi-geostrophic limit. This paper presents a general asymptotic theory for these instabilities. The equations governing the interactions between the strain and the slow bending waves of each vortex column in stratified and rotating fluids are derived for long vertical wavelength and when the two vortices are well separated, i.e. when the radii $R$ of the vortex cores are small compared to the vortex separation distance $b$. These equations have the same form as those obtained for vortex filaments in homogeneous fluids except that the expressions of the mutual-induction and self-induction functions are different. A key difference is that the sign of the self-induction function is reversed compared to homogeneous fluids when the fluid is strongly stratified: $\left|\hat{\Omega}_{\max }\right|<N$ (where $N$ is the Brunt-Väisälä frequency and $\hat{\Omega}_{\max }$ the maximum angular velocity of the vortex) for any vortex profile and magnitude of the planetary rotation. Physically, this means that slow bending waves of a vortex rotate in the same direction as the flow inside the vortex when the fluid is stratified-rotating in contrast to homogeneous fluids. When the stratification is weaker, i.e. $\left|\hat{\Omega}_{\max }\right|>N$, the self-induction function is complex because the bending waves are damped by a viscous critical layer at the radial location where the angular velocity of the vortex is equal to the Brunt-Väisälä frequency.

In contrast to previous theories, which apply only to strongly stratified nonrotating fluids, the present theory is valid for any planetary rotation rate and when the strain is smaller than the Brunt-Väisälä frequency: $\Gamma /\left(2 \pi b^{2}\right) \ll N$, where $\Gamma$ is the vortex circulation. Since the strain is small, this condition is met across a wide range of stratification: from weakly to strongly stratified fluids. The theory is further generalized formally to any basic flow made of an arbitrary number of vortices in stratified and rotating fluids. Viscous and diffusive effects are also taken into account at leading order in Reynolds number when there is no critical layer. In Part 2 (Billant et al., J. Fluid Mech., 2010, doi:10.1017/S002211201000282X), the stability of vortex pairs will be investigated using the present theory and the predictions will be shown to be in very good agreement with the results of direct numerical stability analyses. The existence of the zigzag instability and the distinctive stability properties of vortex pairs in stratified and rotating fluids compared to homogeneous fluids will be demonstrated to originate from the sign reversal of the self-induction function.

Key words: geophysical and geological flows, instability, vortex flows 


\section{Introduction}

A three-dimensional instability, called zigzag instability or tall-column instability, has been observed on co-rotating and counter-rotating columnar vertical vortex pairs in stratified and rotating fluids (Dritschel \& de la Torre Juárez 1996; Billant \& Chomaz 2000a; Otheguy, Billant \& Chomaz 2006a; Otheguy, Chomaz \& Billant 2006b; Deloncle, Billant \& Chomaz 2008; Waite \& Smolarkiewicz 2008). The zigzag instability consists of three-dimensional bending of the vortices with weak core deformations. Ultimately, it generates thin horizontal layers and may explain the layering observed in stratified flows (Riley \& Lelong 2000) and the structure of quasi-geostrophic turbulence (Dritschel, de la Torre Juárez \& Ambaum 1999).

In the case of counter-rotating vortex pairs, the initial evolution of the zigzag instability in strongly stratified fluids (Billant \& Chomaz 2000a) qualitatively resembles that of the Crow instability in homogeneous fluids (Crow 1970), except that the Crow instability bends the vortices symmetrically with respect to the middle plane whereas the zigzag instability is antisymmetric (Billant \& Chomaz 2000a). In the case of co-rotating vortex pairs, the zigzag instability is symmetric (Otheguy et al. $2006 a, b)$ whereas no long-wavelength bending instability occurs in homogeneous fluids (Jimenez 1975). In the latter case, only the elliptic instability has been observed but such instability is of different nature since it distorts the vortex core structure (Meunier \& Leweke 2005; Le Dizès 2008).

The Crow instability of counter-rotating vortex pairs in homogeneous fluid is due to the interaction between the strain that each vortex exerts on its companion and the so-called slow bending modes of each vortex (Crow 1970; Widnall, Bliss \& Zalay 1971). This particular bending mode corresponds to a deflection of the vortex tube with negligible internal deformations and is called 'slow' because its frequency tends to zero in the long-wavelength limit (Leibovich, Brown \& Patel 1986).

In order to prove that the same physical mechanism is at work in the case of the zigzag instability and to provide a complete theory of the zigzag instability in stratified and rotating fluids, the first step is therefore to theoretically describe the dynamics of slow bending waves of a vortex in stratified and rotating fluids in the presence of a companion vortex. This is the subject of the present paper. In a companion paper (Billant et al. 2010) (hereinafter referred to as Part 2), the stability of vortex pairs will be investigated using such theoretical description and the predictions will be shown to fully explain the existence and characteristics of the zigzag instability.

In homogeneous fluids, the Crow instability has been described theoretically by considering vortex filaments (Crow 1970). The vortex filament method is valid for large vortex separation and long-wavelength bending disturbances, and is therefore particularly suited to the Crow instability. This method relies upon the use of the Biot-Savart law to compute the induced motions and upon the cutoff approximation to determine the self-induced motion of the vortices. The latter approximation consists of integrating the Biot-Savart law over all of the vortex except a small segment on either side of the point where the velocity is evaluated. This amounts to take into account the finite size of the vortex cores in order to avoid the logarithmic singularity of the Biot-Savart law. More fundamentally, the vortex filament method is based on the theorems of Helmholtz and Kelvin, which state that vortex lines move as material lines and conserve their circulation in homogeneous and inviscid fluids.

In a stratified and rotating fluid, these theorems are no longer valid, meaning that vortex filament method cannot be used. Nevertheless, the Ertel's theorem states that the potential vorticity is conserved following the motion. In the quasi-geostrophic limit, the potential vorticity and the streamfunction of the flow are related by a 
linear operator relationship so that the horizontal induced motion is also given by a Biot-Savart law. One could therefore consider vortex filaments of potential vorticity in quasi-geostrophic fluids and resort to the same method as Crow (1970). However, a difficulty of this method is that it needs to be completed by using the cutoff approximation. In homogeneous fluids, the choice of the cutoff distance has been justified rigorously by determining the self-induced motion of a single vortex with a large curvature by means of matched asymptotic expansions which take into account the finite size of the vortex core (Widnall et al. 1971; Moore \& Saffman 1972; Leibovich et al. 1986). However, such asymptotic analysis is not very far from directly considering the full problem of two well-separated vortices perturbed by long-wavelength bending disturbances. This is the strategy we have chosen here for a stratified and rotating fluid. A major advantage is that we will be able to carry out the analysis for any Rossby number and over a large range of Froude numbers, i.e. for conditions much wider than those of the quasi-geostrophic regime. Furthermore, the analysis will be conducted for any vortex pair of arbitrary relative strength and any velocity profile of the individual vortices. Viscous and diffusive effects will also be taken into account at leading order. Thus, we shall extend in many directions the previous theoretical analyses of the zigzag instability which have been performed only in strongly stratified non-rotating inviscid fluids and for the specific cases of the Lamb-Chaplygin counter-rotating vortex pair (Billant \& Chomaz 2000b) and two equal-strength co-rotating Lamb-Oseen vortices (Otheguy, Billant \& Chomaz 2007). These analyses have shown that the zigzag instability for these two basic flows can be interpreted as a breaking of translational or rotational invariances of the global basic flow for long vertical wavelength in a strongly stratified fluid. However, such an approach is difficult to generalize to weakly stratified-rotating fluids or to other basic flows. In contrast, the present theory has a general formalism which enables its use for any basic flow with an arbitrary number of vortices.

The paper is organized as follows. We first compute in $\S 2.2$ a basic state made of two vertical vortices when they are well separated, i.e. when the ratio of separation distance $b$ to vortex radius $R$ is large: $b / R \gg 1$. After having non-dimensionalized the governing equations in $\S 2.3$, the three-dimensional stability analysis of this basic state is analysed asymptotically in $\S 3$ for long-wavelength bending perturbations. The resulting stability equations describe the coupling between the strain and the slow long-wavelength bending disturbances of each vortex. They happen to have the same form as those obtained by Crow (1970), Jimenez (1975) and Bristol et al. (2004) in homogeneous fluids using vortex filaments except that the explicit forms of the mutual-induction and self-induction functions are different in stratified and rotating fluids. The properties of the mutual-induction and self-induction functions in stratified and rotating fluids are analysed and compared to their counterparts in homogeneous fluids in $\S 4$. Finally, the stability equations are generalized to any number of vortices in $\S 5$.

\section{Stability problem}

\subsection{Governing equations}

We consider a rotating, stably stratified fluid under the Boussinesq approximation. The equations of momentum, continuity and density conservation read

$$
\frac{\mathrm{D} \hat{\boldsymbol{u}}}{\mathrm{D} \hat{t}}+2 \Omega_{b} \boldsymbol{e}_{z} \times \hat{\boldsymbol{u}}=-\frac{1}{\rho_{0}} \nabla \hat{p}-\frac{g \hat{\rho}}{\rho_{0}} \boldsymbol{e}_{z}+v \Delta \hat{\boldsymbol{u}},
$$




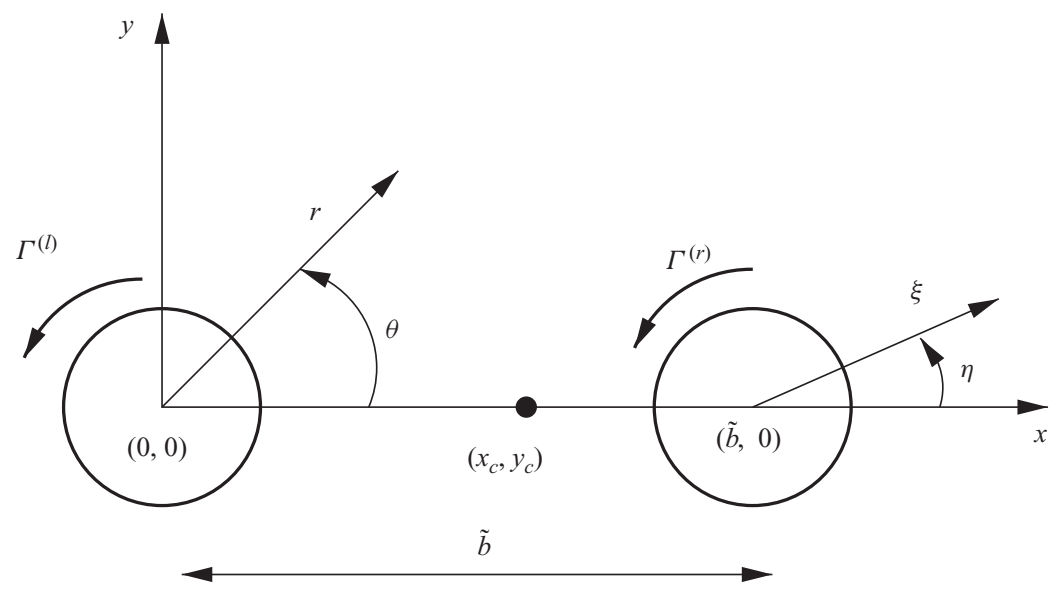

FiguRE 1. Sketch of the vortex pair in the frame of reference where it is steady. $(x, y)$ are the Cartesian coordinates centred on the left vortex. $(r, \theta)$ and $(\xi, \eta)$ are the cylindrical coordinates centred on the left and the right vortex, respectively. The rotation centre of the vortex pair is located at $\left(x_{c}, y_{c}\right)$.

$$
\begin{gathered}
\nabla \cdot \hat{\boldsymbol{u}}=0, \\
\frac{\mathrm{D} \hat{\rho}}{\mathrm{D} \hat{t}}+\frac{\partial \bar{\rho}}{\partial \hat{z}} \hat{u}_{z}=D \Delta \hat{\rho},
\end{gathered}
$$

with $\hat{\boldsymbol{u}}$ being the velocity, $\Omega_{b}$ the rotation rate about the vertical axis, $\boldsymbol{e}_{z}$ the vertical unit vector, $\hat{u}_{z}$ the vertical velocity, $\hat{p}$ the pressure, $g$ the gravity, $v$ the viscosity, $\Delta$ the Laplacian and $D$ is the diffusivity of the stratifying agent. The total density field $\rho_{t}$ has been decomposed as $\rho_{t}(\hat{\boldsymbol{x}}, \hat{t})=\rho_{0}+\bar{\rho}(\hat{z})+\hat{\rho}(\hat{\boldsymbol{x}}, \hat{t})$, with $\rho_{0}$ being a constant reference density, $\bar{\rho}(\hat{z})$ a linear mean density profile and $\hat{\rho}(\hat{\boldsymbol{x}}, \hat{\boldsymbol{t}})$ a perturbation density. The Brunt-Väisälä frequency $N=\sqrt{-\left(g / \rho_{0}\right) \partial \bar{\rho} / \partial \hat{z}}$, measuring the density gradient, is assumed to be constant.

\subsection{The basic flow}

We consider two columnar vertical vortices of circulation $\Gamma^{(l)}$ and $\Gamma^{(r)}$ separated by a distance $b$ in the frame of reference rotating at rate $\Omega_{b}$ (figure 1). The fluid is assumed inviscid and non-diffusive. When the radii $R^{(l)}$ and $R^{(r)}$ of each vortex are small compared to $b$, the vortices rotate around each other at rate $f=\left(\Gamma^{(l)}+\Gamma^{(r)}\right) /\left(2 \pi b^{2}\right)$ exactly like two point vortices and each vortex adapts to the strain field generated by the other vortex. Moore \& Saffman (1975) (see also Saffman 1992, Rossi 2000 and Le Dizès \& Laporte 2002) have shown that this adaptation can be computed asymptotically when the two vortices are well separated. For clarity, we briefly repeat their analysis below.

We first switch from the planetary frame rotating at absolute angular velocity $\Omega_{b}$ to the reference frame rotating at absolute angular velocity $f+\Omega_{b}$, where the vortex pair is steady. We also make the problem dimensionless by using the quantities of the vortex labelled with superscript $(l)$. Time is non-dimensionalized by $2 \pi R^{(l)^{2}} / \Gamma^{(l)}$ and horizontal length by $R^{(l)}$ (The corresponding non-dimensional variables will be denoted without a hat.) The non-dimensional circulation of the vortex labelled with superscript $(r)$ is $\tilde{\Gamma}=\Gamma^{(r)} / \Gamma^{(l)}$ and its non-dimensional radius $\tilde{R}=R^{(r)} / R^{(l)}$. The nondimensional separation distance is $\tilde{b}=b / R^{(l)}$ and the non-dimensional rate of rotation 
of the pair is $\tilde{f} \epsilon$, where $\tilde{f}=1+1 / \tilde{\Gamma}$ and

$$
\epsilon=\tilde{\Gamma} / \tilde{b}^{2}
$$

is the non-dimensional strain.

The centre of the vortex labelled with the superscript $(l)$ is chosen to be located on the left at $(x=0, y=0)$ and the centre of the vortex labelled with the superscript $(r)$ is on the right at $(x=\tilde{b}, y=0)$ (figure 1). The rotation centre of the vortex pair is at $\left(x_{c}=\tilde{b} / \tilde{f}, y_{c}=0\right)$. The basic flow $\boldsymbol{U}_{b}$ can be written in term of a streamfunction: $\boldsymbol{U}_{b}=-\nabla \times \psi_{b} \boldsymbol{e}_{z}$, which can be decomposed as

$$
\psi_{b}=\psi_{b}^{(l)}+\psi_{b}^{(r)}+\psi_{f}
$$

where $\psi_{b}^{(l)}$ and $\psi_{b}^{(r)}$ are the streamfunctions corresponding to each vortex and $\psi_{f}=-\tilde{f} \epsilon\left[\left(x-x_{c}\right)^{2}+\left(y-y_{c}\right)^{2}\right] / 2$ is the streamfunction of the solid-body rotation due to the rotation of the frame of reference relative to the planetary reference frame. Note that in the limit $\tilde{\Gamma}=-1$, we have $\psi_{f}=\tilde{b} \in x$ up to an arbitrary constant, meaning that the co-moving reference frame no longer rotates but translates along the $y$-axis. The streamfunction of each vortex can be decomposed as follows:

$$
\psi_{b}^{(i)}=\psi_{a}^{(i)}+\psi_{d}^{(i)}
$$

for $i=\{l, r\}$, where $\psi_{a}^{(i)}$ is the streamfunction of the vortex $(i)$ as if it were alone and $\psi_{d}^{(i)}$ corresponds to its adaptation to the strain induced by the other vortex.

The ratio between the vorticity of each vortex core and the strain induced by its companion is $O(1 / \epsilon)$. Therefore, when the two vortices are well separated, i.e. $\tilde{b} \gg 1$, the streamfunctions $\psi_{d}^{(l)}$ and $\psi_{d}^{(r)}$ are $O(\epsilon) \ll 1$ and can be computed asymptotically. Let us consider the vortex $(l)$. The condition that the flow is steady is

$$
J\left(\psi_{b}, \Delta \psi_{b}\right)=\frac{\partial \psi_{b}}{\partial x} \frac{\partial \Delta \psi_{b}}{\partial y}-\frac{\partial \psi_{b}}{\partial y} \frac{\partial \Delta \psi_{b}}{\partial x}=0,
$$

where $J$ denotes the Jacobian. If we focus on the region close to the core of the left vortex, this gives at zeroth order in $\epsilon$

$$
J\left(\psi_{a}^{(l)}, \Delta \psi_{a}^{(l)}\right)=0 .
$$

This equation is satisfied by any axisymmetric vortex with streamfunction $\psi_{a}^{(l)}(r, \theta) \equiv$ $\psi_{a}^{(l)}(r)$, with $(r, \theta)$ being the cylindrical coordinates centred on the left vortex. At first order in $\epsilon$, (2.7) gives

$$
J\left(\psi_{a}^{(l)}, \Delta\left(\psi_{d}^{(l)}+\psi_{f}+\psi_{b}^{(r)}\right)\right)+J\left(\psi_{d}^{(l)}+\psi_{f}+\psi_{b}^{(r)}, \Delta \psi_{a}^{(l)}\right)=0 .
$$

This equation can be simplified using the fact that the streamfunction $\psi_{b}^{(r)}$ of the right vortex tends to the one of a point vortex in the neighbourhood of the left vortex (i.e. $x-\tilde{b} \gg \tilde{R}$ and $y \gg \tilde{R})$ :

$$
\psi_{b}^{(r)} \sim \psi_{a}^{(r)} \sim \frac{\tilde{\Gamma}}{2} \ln \left((x-\tilde{b})^{2}+y^{2}\right)=\frac{\tilde{\Gamma}}{2}\left[\ln \tilde{b}^{2}-2 \frac{r}{\tilde{b}} \cos \theta-\frac{r^{2}}{\tilde{b}^{2}} \cos 2 \theta+O\left(\frac{1}{\tilde{b}^{3}}\right)\right],
$$

where we have anticipated that $\psi_{d}^{(r)}$ vanishes outside the core of the right vortex. After an integration, (2.9) then leads to the following equation for $\psi_{d}^{(l)}$ :

$$
\frac{\partial \psi_{a}^{(l)}}{\partial r} \Delta \psi_{d}^{(l)}-\frac{\partial \Delta \psi_{a}^{(l)}}{\partial r} \psi_{d}^{(l)}=-\frac{\partial \Delta \psi_{a}^{(l)}}{\partial r} \epsilon\left(\frac{\tilde{f}}{2} r^{2}+\frac{1}{2} r^{2} \cos 2 \theta\right)+\mathscr{G}(r),
$$


where $\mathscr{G}(r)$ is an arbitrary function. In order that $\psi_{d}^{(l)} \rightarrow 0$ as $r \rightarrow \infty$ and be non-singular at $r=0$, one has to impose $\mathscr{G}(r)=\tilde{f} \epsilon\left(r^{2} / 2\right) \partial \Delta \psi_{a}^{(l)} / \partial r$. This implies that the streamfunction $\psi_{d}^{(l)}$ is independent of the rotation rate $\tilde{f}$ of the vortex pair in contrast to the analysis of Le Dizès \& Laporte (2002). The reason for this difference is that the latter authors assume $\psi_{f}=O(1)$ whereas $\psi_{f}=O(\epsilon)$ in the present situation.

The solution of (2.11) is then of the form $\psi_{d}^{(l)}=(\epsilon / 2) h(r) \cos 2 \theta$, where the function $h$ can be determined numerically with the boundary conditions $h(r) \rightarrow 0$ as $r \rightarrow$ $\infty$ and $h(r) \propto r^{2}$ as $r \rightarrow 0$. As shown by Moore \& Saffman (1975) (see also Saffman 1992 and Le Dizès \& Laporte 2002), the streamfunction $\psi_{d}^{(l)}$ corresponds to an enhancement of the strain in the core of the vortex due to the interaction between strain and vorticity. This is the so-called internal strain which vanishes rapidly outside the vortex core: $h(r) \rightarrow 0$ as $r \rightarrow \infty$. The latter condition is in fact the only property needed in the asymptotic analysis of $\S 3$. We shall see that the explicit knowledge of the function $h(r)$ is not necessary except when a critical layer exists.

In conclusion, the basic flow near the vortex labelled $(l)$ can be written for $r \ll \tilde{b}:$

$$
\boldsymbol{U}_{b}=\frac{\partial \psi_{a}^{(l)}}{\partial r} \boldsymbol{e}_{\theta}+\epsilon \boldsymbol{U}_{s}+O\left(\frac{1}{\tilde{b}^{3}}\right)
$$

with $\boldsymbol{U}_{s}=-\nabla \times \psi_{s} \boldsymbol{e}_{z}$, where

$$
\psi_{s}=-\frac{1}{2}\left[\left(r^{2}-h(r)\right) \cos 2 \theta+\tilde{f} r^{2}\right]
$$

corresponds to a non-uniform rotating straining flow. Similar expressions can be obtained near the right vortex. Finally, far from the two vortex cores, the streamfunction $\psi_{b}$ of the base flow tends to the streamfunction of two points vortices

$$
\psi_{b}=\frac{\tilde{\Gamma}}{2} \ln \left((x-\tilde{b})^{2}+y^{2}\right)+\frac{1}{2} \ln \left(x^{2}+y^{2}\right)-\frac{\tilde{f} \epsilon}{2}\left[\left(x-x_{c}\right)^{2}+\left(y-y_{c}\right)^{2}\right] .
$$

\subsection{Scaling analysis}

As mentioned before, the horizontal length unit is taken as the vortex radius $R^{(l)}$ of the left vortex and the time unit is chosen as $2 \pi R^{(l)^{2}} / \Gamma^{(l)}$. Accordingly, the horizontal velocity $\hat{\boldsymbol{u}}_{h}$ is non-dimensionalized by $\Gamma^{(l)} /\left(2 \pi R^{(l)}\right)$ and the pressure by $\rho_{0} \Gamma^{(l)^{2}} /\left(2 \pi R^{(l)}\right)^{2}$.

Since we shall be mostly interested by stratified flows for which the horizontal Froude number,

$$
F_{h}^{(l)}=\frac{\left|\Gamma^{(l)}\right|}{2 \pi R^{(l)^{2} N}},
$$

will be small, it is convenient to scale the vertical coordinate by $F_{h}^{(l)} R^{(l)}$, the vertical velocity $\hat{u}_{z}$ by $F_{h}^{(l)} \Gamma^{(l)} /\left(2 \pi R^{(l)}\right)$ and density fluctuations $\hat{\rho}$ by $\rho_{0} \Gamma^{(l)} N /\left(g 2 \pi R^{(l)}\right)$ following Billant \& Chomaz (2001). 
We now write the non-dimensional governing equations in the reference frame rotating at non-dimensional rate $\tilde{f} \epsilon+1 /\left(2 R o^{(l)}\right)$ where the base flow is steady:

$$
\begin{gathered}
\frac{\partial \boldsymbol{u}_{h}}{\partial t}+\boldsymbol{u}_{h} \cdot \nabla_{h} \boldsymbol{u}_{h}+u_{z} \frac{\partial \boldsymbol{u}_{h}}{\partial z}+\left(2 \tilde{f} \epsilon+\frac{1}{R o^{(l)}}\right) \boldsymbol{e}_{z} \times \boldsymbol{u}_{h}=-\nabla_{h} p+\frac{\delta_{\Gamma}}{R e^{(l)}} \Delta_{s} \boldsymbol{u}_{h} \\
F_{h}^{(l)^{2}}\left[\frac{\partial u_{z}}{\partial t}+\boldsymbol{u}_{h} \cdot \nabla_{h} u_{z}+u_{z} \frac{\partial u_{z}}{\partial z}\right]=-\frac{\partial p}{\partial z}-\rho+F_{h}^{(l)^{2}} \frac{\delta_{\Gamma}}{R e^{(l)}} \Delta_{s} u_{z} \\
\nabla_{h} \cdot \boldsymbol{u}_{h}+\frac{\partial u_{z}}{\partial z}=0 \\
\frac{\partial \rho}{\partial t}+\boldsymbol{u}_{h} \cdot \nabla_{h} \rho+u_{z} \frac{\partial \rho}{\partial z}=u_{z}+\frac{\delta_{\Gamma}}{R e^{(l)} S c} \Delta_{s} \rho
\end{gathered}
$$

where the non-dimensional variables are denoted without a hat, $\nabla_{h}$ is the horizontal gradient, $\Delta_{s}=\Delta_{h}+F_{h}^{(l)}{ }^{-2} \partial^{2} / \partial z^{2}$, with $\Delta_{h}$ being the horizontal Laplacian, $\delta_{\Gamma}=\operatorname{sgn}\left(\Gamma^{(l)}\right), S c=v / D$ is the Schmidt number and

$$
R o^{(l)}=\frac{\Gamma^{(l)}}{4 \Omega_{b} \pi R^{(l)^{2}}}, \quad R e^{(l)}=\frac{\left|\Gamma^{(l)}\right|}{2 \pi \nu},
$$

are the Rossby number and Reynolds numbers. Note that the Froude, Rossby and Reynolds numbers defined in Otheguy et al. $(2006 a, b, 2007)$ are twice those used in the present paper. It should be emphasized that this non-dimensionalization is only a convenient way to rewrite the equations for the study of strongly stratified flows but (2.16)-(2.19) remain valid for any Froude number.

Correspondingly, the Froude number $F_{h}^{(r)}$, the Rossby number $R o^{(r)}$ and the Reynolds number $R e^{(r)}$ for the right vortex are defined as (2.15) and (2.20) with the superscript $(l)$ replaced by $(r)$.

\section{Asymptotic three-dimensional stability analysis}

We now subject the basic flow to infinitesimal three-dimensional perturbations denoted by a tilde:

$$
[\boldsymbol{u}, p, \rho](x, y, z, t)=\left[\boldsymbol{U}_{b}, P_{b}, 0\right](x, y)+\operatorname{Re}\left([\tilde{\boldsymbol{u}}, \tilde{p}, \tilde{\rho}](x, y, t) \mathrm{e}^{\mathrm{i} k z}\right),
$$

where $k$ is the non-dimensional vertical wavenumber and $\mathrm{Re}$ denotes the real part. The corresponding dimensional wavenumber is $\hat{k}=k /\left(F_{h}^{(l)} R^{(l)}\right)$, due to the nondimensionalization of $\S 2.3$. In the main part of the analysis, we shall consider the equations of an inviscid and non-diffusive fluid except if they become singular. Viscous effects will then be re-introduced into the problem in order to smooth the singularity. The viscous and diffusive effects when there is no singularity will also be considered in Appendix D. The non-dimensional linearized equations (2.16)-(2.19), governing the disturbances for $R e^{(l)}=\infty$, are

$$
\begin{aligned}
\frac{\partial \tilde{\boldsymbol{u}}_{h}}{\partial t}+\boldsymbol{U}_{b} \cdot \nabla_{h} \tilde{\boldsymbol{u}}_{h}+\tilde{\boldsymbol{u}}_{h} \cdot \nabla_{h} \boldsymbol{U}_{b}+\left(2 \tilde{f} \epsilon+\frac{1}{R o^{(l)}}\right) \boldsymbol{e}_{z} \times \tilde{\boldsymbol{u}}_{h}=-\nabla_{h} \tilde{p} \\
F_{h}^{(l)^{2}}\left(\frac{\partial \tilde{u}_{z}}{\partial t}+\boldsymbol{U}_{b} \cdot \nabla_{h} \tilde{u}_{z}\right)=-\mathrm{i} k \tilde{p}-\tilde{\rho} \\
\nabla_{h} \cdot \tilde{\boldsymbol{u}}_{h}+\mathrm{i} k \tilde{u}_{z}=0 \\
\frac{\partial \tilde{\rho}}{\partial t}+\boldsymbol{U}_{b} \cdot \nabla_{h} \tilde{\rho}=\tilde{u}_{z} .
\end{aligned}
$$


It will also be convenient to use the equation for the vertical vorticity $\tilde{\zeta}=\left(\nabla \times \tilde{\boldsymbol{u}}_{h}\right) \boldsymbol{e}_{z}$ :

$$
\frac{\partial \tilde{\zeta}}{\partial t}+\boldsymbol{U}_{b} \cdot \nabla_{h} \tilde{\zeta}+\tilde{\boldsymbol{u}}_{h} \cdot \nabla_{h} \Delta \psi_{b}-\mathrm{i} k\left(\Delta \psi_{b}+\frac{1}{R o^{(l)}}+2 \tilde{f} \epsilon\right) \tilde{u}_{z}=0
$$

The stability problem will be solved asymptotically for well-separated vortices, i.e. a small strain, $\epsilon=\tilde{\Gamma} / \tilde{b}^{2} \ll 1$, and for long-wavelength disturbances such that

$$
\mu \equiv \frac{k}{\min \left(1,\left|R o^{(l)}\right|\right) \max \left(1, F_{h}^{(l)}\right)} \ll 1 .
$$

This condition comes from the fact that the leading three-dimensional terms for a given Froude number scale as $k^{2}$ for a large Rossby number and as $k^{2} / R o^{(l)^{2}}$ for a small Rossby number. Alternatively, if the Rossby number is fixed, the leading three-dimensional terms are proportional to $k^{2}$ for $F_{h}^{(l)}<O(1)$ and to $\left(k / F_{h}^{(l)}\right)^{2}$ for large Froude number. The condition (3.7) therefore ensures that the three-dimensional effects are always small whatever be the Rossby and Froude numbers. In addition, we impose the scaling $\mu^{2}=O(\epsilon)$ to ensure that three-dimensional effects and strain effects come up at the same order and can interact. This assumption is equivalent to state that the rescaled wavelength of the disturbances is of the same order as the separation distance $1 / \mu=O(\tilde{b})$. In practice, the final equations will be valid for any $\mu \tilde{b}$ as long as the two fundamental assumptions $\mu \ll 1$ and $\tilde{b} \gg 1$ are fulfilled.

We first consider the perturbations in the vicinity of the core of the left vortex where the base flow is given by (2.12). It turns out that the solution in this inner region is not uniformly asymptotic for large radius. The solution in an outer region will therefore be determined next and matched to the inner solution. The perturbations are written in asymptotic series with $\epsilon$ as the main expansion parameter:

$$
\begin{aligned}
\tilde{\boldsymbol{u}}_{h} & =\tilde{\boldsymbol{u}}_{h 0}+\epsilon \tilde{\boldsymbol{u}}_{h 1}+\cdots, \\
\tilde{p} & =\tilde{p}_{0}+\epsilon \tilde{p}_{1}+\cdots, \\
\tilde{u}_{z} & =k\left(\tilde{u}_{z 0}+\epsilon \tilde{u}_{z 1}+\cdots\right), \\
\tilde{\rho} & =k\left(\tilde{\rho}_{0}+\epsilon \tilde{\rho}_{1}+\cdots\right) .
\end{aligned}
$$

The terms of order $\mu^{2}$ will be included in the $O(\epsilon)$ order. For convenience, the vertical velocity $\tilde{u}_{z}$ and density $\tilde{\rho}$ have been scaled by $k$. We also introduce a slow time scale of order $O\left(\epsilon, \mu^{2}\right): T=\epsilon t$.

\subsection{Inner region of the left vortex}

\subsubsection{Zeroth-order problem}

At zeroth order, the base flow (2.12) simply corresponds to an axisymmetric isolated vortex and the perturbations are governed by

$$
\begin{aligned}
\frac{\partial \tilde{u}_{r 0}}{\partial t}+\Omega \frac{\partial \tilde{u}_{r 0}}{\partial \theta}-\left(2 \Omega+\frac{1}{R o^{(l)}}\right) \tilde{u}_{\theta 0} & =-\frac{\partial \tilde{p}_{0}}{\partial r}, \\
\frac{\partial \tilde{u}_{\theta 0}}{\partial t}+\Omega \frac{\partial \tilde{u}_{\theta 0}}{\partial \theta}+\left(\zeta+\frac{1}{R o^{(l)}}\right) \tilde{u}_{r 0} & =-\frac{1}{r} \frac{\partial \tilde{p}_{0}}{\partial \theta}, \\
F_{h}^{(l)}\left(\frac{\partial \tilde{u}_{z 0}}{\partial t}+\Omega \frac{\partial \tilde{u}_{z 0}}{\partial \theta}\right) & =-\mathrm{i} \tilde{p}_{0}-\tilde{\rho}_{0},
\end{aligned}
$$




$$
\begin{aligned}
\frac{1}{r} \frac{\partial r \tilde{u}_{r 0}}{\partial r}+\frac{1}{r} \frac{\partial \tilde{u}_{\theta 0}}{\partial \theta} & =0, \\
\frac{\partial \tilde{\rho}_{0}}{\partial t}+\Omega \frac{\partial \tilde{\rho}_{0}}{\partial \theta} & =\tilde{u}_{z 0},
\end{aligned}
$$

where $\Omega(r)=(1 / r) \partial \psi_{a}^{(l)} / \partial r$ and $\zeta(r)=\Delta \psi_{a}^{(l)}$ are the basic angular velocity and vertical vorticity of the left vortex at leading order.

The horizontal velocity of the perturbation obeys purely two-dimensional equations. Since the basic flow at leading order is axisymmetric, we can seek the solution in the form $\tilde{\boldsymbol{u}}_{h 0}=-\nabla \times\left(\tilde{\psi}_{0} \boldsymbol{e}_{z}\right)$ with $\tilde{\psi}_{0}=\varphi_{0}(r) \exp \left(\mathrm{i} m \theta-\mathrm{i} \omega_{0} t\right)$. Then, (3.12)-(3.13) give

$$
\frac{\partial^{2} \varphi_{0}}{\partial r^{2}}+\frac{1}{r} \frac{\partial \varphi_{0}}{\partial r}-\left[\frac{m^{2}}{r^{2}}+\frac{\zeta^{\prime}}{r\left(\Omega-\omega_{0}\right)}\right] \varphi_{0}=0,
$$

where the prime denotes differentiation with respect to $r$. Here, we shall consider bending waves of the vortex, i.e. waves with azimuthal wavenumbers $|m|=1$. In this case, the general solution of (3.17) can be found for any angular velocity profile $\Omega$ (Michalke \& Timme 1967; Widnall et al. 1971):

$$
\varphi_{0}=\operatorname{Cr}\left(\Omega-\omega_{0}\right)+\operatorname{Dr}\left(\Omega-\omega_{0}\right) \int \frac{\mathrm{d} r}{r^{3}\left(\Omega-\omega_{0}\right)^{2}},
$$

where $C$ and $D$ are constants. The second solution is singular at $r=0$ and therefore one has to set $D=0$. The first solution is non-singular at $r=0$ since $\Omega(0)$ is assumed to be finite. However, this solution is unbounded as $r \rightarrow \infty$ when $\omega_{0} \neq 0$ since $\varphi_{0} \sim C\left(1 / r-\omega_{0} r\right)$ for $r \gg 1$. The matching with a decaying outer solution is possible only if $\omega_{0}=0$.

Therefore, the total zero-order streamfunction can be written in the form

$$
\tilde{\psi}_{0}=r \Omega\left(C_{+}^{(l)}(T) \mathrm{e}^{\mathrm{i} \theta}+C_{-}^{(l)}(T) \mathrm{e}^{-\mathrm{i} \theta}\right),
$$

where $C_{+}^{(l)}$ and $C_{-}^{(l)}$ are the amplitudes of the waves with azimuthal wavenumbers $m=1$ and $m=-1$. They are assumed to be function of the slow time scale $T=\epsilon t$. This remarkable solution derives from the translational invariances. Indeed, (3.19) can be rewritten as

$$
\tilde{\psi}_{0}=-\Delta x^{(l)} \frac{\partial \psi_{a}^{(l)}}{\partial x}-\Delta y^{(l)} \frac{\partial \psi_{a}^{(l)}}{\partial y},
$$

with the relations

$$
\begin{aligned}
& \Delta x^{(l)}=-C_{+}^{(l)}-C_{-}^{(l)}, \\
& \Delta y^{(l)}=-\mathrm{i}\left(C_{+}^{(l)}-C_{-}^{(l)}\right) .
\end{aligned}
$$

If we add the infinitesimal perturbation (3.20) to the streamfunction of the basic flow $\psi_{a}^{(l)}$, we have

$$
\psi_{a}^{(l)}(x, y)+\operatorname{Re}\left(\tilde{\psi}_{0} \mathrm{e}^{\mathrm{i} k z}\right) \sim \psi_{a}^{(l)}\left(x-\operatorname{Re}\left(\Delta x^{(l)} \mathrm{e}^{\mathrm{i} k z}\right), y-\operatorname{Re}\left(\Delta y^{(l)} \mathrm{e}^{\mathrm{i} k z}\right)\right),
$$

meaning that the solution $\tilde{\psi}_{0}$ simply corresponds to a displacement of $\operatorname{Re}\left(\Delta x^{(l)} \exp (\mathrm{i} k z)\right)$ and $\operatorname{Re}\left(\Delta y^{(l)} \exp (\mathrm{i} k z)\right)$ of the left vortex as a whole in the $x$ and $y$ directions. Since $k$ is assumed to be small but non-zero, the whole vortex tube is sinusoidally bent along the vertical without deformations in the horizontal plane. Weak radial deformations will, however, be found at the next orders. These waves are generally called 'slow bending waves' because their frequency is zero in the limit $k=0$ 
(Leibovich et al. 1986). They are different from other bending waves which may exist for finite vertical wavenumber or finite frequency and which have a different radial structure.

The corresponding vertical vorticity, pressure and vertical velocity given by (3.12)(3.16) are

$$
\begin{aligned}
\tilde{\zeta}_{0} & =\zeta^{\prime}\left(C_{+}^{(l)} \mathrm{e}^{\mathrm{i} \theta}+C_{-}^{(l)} \mathrm{e}^{-\mathrm{i} \theta}\right), \\
\tilde{p}_{0} & =r \Omega\left(\Omega+R o^{(l)^{-1}}\right)\left(C_{+}^{(l)} \mathrm{e}^{\mathrm{i} \theta}+C_{-}^{(l)} \mathrm{e}^{-\mathrm{i} \theta}\right), \\
\tilde{u}_{z 0} & =W_{+} C_{+}^{(l)} \mathrm{e}^{\mathrm{i} \theta}-W_{-} C_{-}^{(l)} \mathrm{e}^{-\mathrm{i} \theta},
\end{aligned}
$$

where

$$
W_{+}=W_{-}=W^{i} \equiv \Omega^{2} r \frac{\Omega+R o^{(l)}-1}{1-F_{h}^{(l)^{2}} \Omega^{2}} .
$$

The expression for the amplitude $W^{i}$ of the vertical velocity is valid for all $r$ when $F_{h}^{(l)}<1 / \Omega_{\max }$, where $\Omega_{\max }$ is the maximum non-dimensional angular velocity of the left vortex. (Note that we assume that $\Omega$ decreases monotonically with $r$ as observed for most vortex profiles.) This condition is equivalent to $\left|\hat{\Omega}_{\max }\right| / N<1$, where $\hat{\Omega}_{\max }$ is the corresponding maximum dimensional angular velocity. When $F_{h}^{(l)}>1 / \Omega_{\max }$, the vertical velocity amplitude (3.27) presents a singularity at the radius $r_{c}$, where $\Omega\left(r_{c}\right)=1 / F_{h}^{(l)}$. Such singularity can be understood as a resonance when the local Doppler shifted frequency of the slow bending mode, i.e. $-\hat{\omega}_{0}+m \hat{\Omega}= \pm \hat{\Omega}$ at leading order and in dimensional form, is equal to the Brunt-Väisäilä frequency. A similar singularity occurs in the case of a slightly tilted columnar axisymmetric vortex in a stratified fluid (Boulanger, Meunier \& Le Dizès 2007). Indeed, the inclination of the vortex forces a vertical velocity and density fields similar to those of the present zero-order perturbation. Near this singularity, the diffusive effects and the terms of order $O(\epsilon)$, namely the advection of the perturbation by the straining flow $\boldsymbol{U}_{s}$ and the evolution of the perturbation on the slow time $T$, should be re-incorporated in (3.14) and (3.16), since they are no longer small compared to the leading-order terms. Nonlinear effects cannot come into play in the singular region since we are in the framework of a linear stability analysis. The structure of this critical layer is analysed in Appendix A following Boulanger et al. (2007). It is shown that the vertical velocity amplitudes $W_{+}$and $W_{-}$near $r_{c}$ become

$$
W_{ \pm}=W_{ \pm}^{v} \equiv \pm \mathrm{i} R e^{(l)^{1 / 3}} \Omega_{c} r_{c} \pi \Lambda \frac{\Omega_{c}+R o^{(l)}-1}{2 F_{h}^{(l)^{2}} \Omega_{c}^{\prime}} \mathrm{Hi}\left( \pm \mathrm{i} \Lambda x_{ \pm}\right)+O\left(\epsilon R e^{(l)^{1 / 3}}\right),
$$

where the subscript $c$ indicates the value taken at $r_{c}, \Lambda=-\operatorname{sgn}\left(\Gamma^{(l)}\right)\left(2 \Omega_{c}^{\prime} /(1+1 / S c)\right)^{1 / 3}$, Hi is the Scorer's function (Abramowitz \& Stegun 1965; Drazin \& Reid 1981; Gil, Segura \& Temme 2002) and

$$
x_{ \pm}=R e^{(l)^{1 / 3}}\left[r-r_{c}-\frac{\epsilon}{2 \Omega_{c}}\left(r_{c}-\frac{h_{c}}{r_{c}}\right) \cos 2 \theta-\frac{\epsilon}{\Omega_{c}^{\prime}}\left(\tilde{f} \pm \mathrm{i} \frac{\partial \ln C_{ \pm}^{(l)}}{\partial T}\right)\right] .
$$

This solution matches the inviscid solution (3.27) away from $r_{c}$ since $\mathrm{Hi}(\xi) \sim-1 /(\pi \xi)$ for $\xi \rightarrow \infty$ with $|\arg (\xi)|>\pi / 3$. Note that $W_{ \pm}$are complex conjugates of one another when $\partial \ln C_{+}^{(l)} / \partial T=\partial \ln C_{-}^{(l)} / \partial T=\sigma$ and the growth rate $\sigma$ is purely real. 

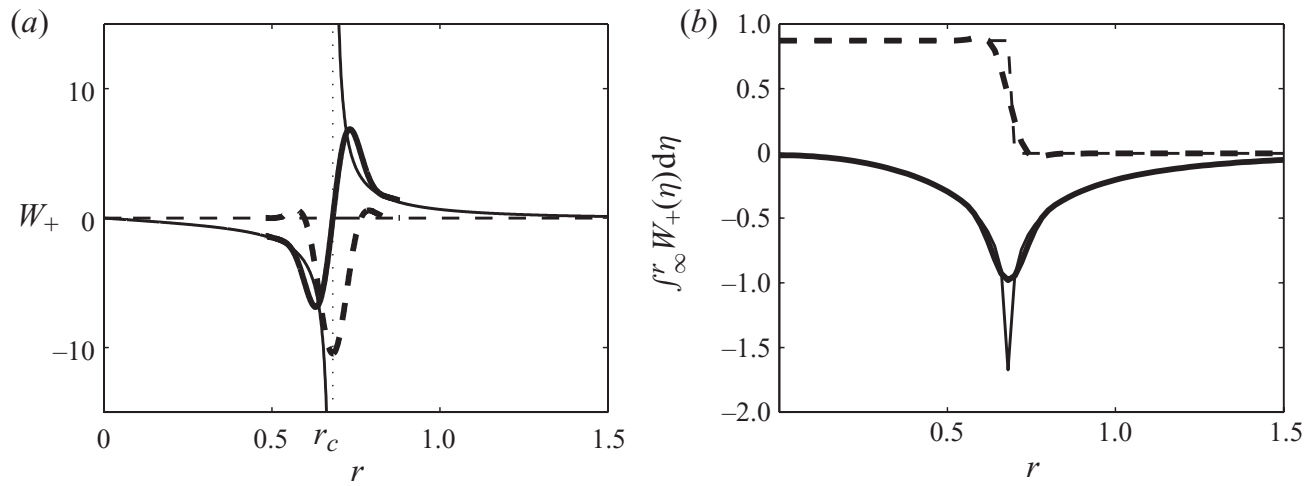

FIGURE 2. Vertical-velocity amplitude $W_{+}(a)$ and integral $\int_{\infty}^{r} W_{+}(\eta) \mathrm{d} \eta(b)$ as a function of $r$ for $F_{h}=1.25, R o=\infty, R e=50000, S c=1$ and $\epsilon=0$ for the Lamb-Oseen vortex (4.7). The real and imaginary parts are shown by solid and dashed lines, respectively. In $(a)$, the thin lines show the inviscid formula (3.27) and the bold lines show the viscous solution (3.28). The dotted line shows the location of the singularity $r_{c}=0.681$. In $(b)$, the thin lines have been plotted by integrating the inviscid formula (3.27) in the complex plane according to the rule (3.38). The bold lines show the result of the integration of the composite formula (3.30) on the real axis.

Figure 2(a) illustrates one example of the velocity amplitude $W_{+}$when a critical point exists. The inviscid solution (3.27) is represented by thin lines and the viscous solution (3.28) for $R e^{(l)}=50000$ and $\epsilon=0$ is shown by bold lines. We can see that the viscous solution smoothes the singularity and perfectly matches the inviscid solution away from the critical radius. It is noteworthy that the viscous solution has an imaginary part (bold dashed line in figure $2 a$ ) in contrast to the inviscid solution.

The critical-layer solution (3.28) is similar to the purely viscous solution derived by Boulanger et al. (2007) except that two additional features are taken into account: the elliptical shape of the vortex and the slow evolution of $C_{ \pm}^{(l)}$ (the last term of (3.29)). Since these two effects are of order $O(\epsilon)$ whereas viscous effect scales as $R e^{(l)^{1 / 3}}$, two different regimes are possible depending upon the number $\operatorname{Re}^{(l)} \epsilon^{3}$. When $R e^{(l)} \epsilon^{3} \ll 1$, i.e. for moderate Reynolds number or very small strain, the terms $O(\epsilon)$ are negligible. The typical amplitude of the vertical velocity in the critical layer then scales as $R e^{(l)^{1 / 3}}$ and the typical size of the critical layer is $R e^{(l)^{-1 / 3}}$, as found by Boulanger et al. (2007). In contrast, for higher Reynolds number, $R e^{(l)} \epsilon^{3} \gg 1$, the terms $O(\epsilon)$ cannot be neglected. The solution (3.28) shows that the critical layer is then concentrated around the elliptic streamline whose mean radius is $r=r_{c}$. Furthermore, if $C_{+}^{(l)}$ (respectively $C_{-}^{(l)}$ ) has a growth rate with a real part of the same sign as $\Gamma^{(r)}$ so that the dimensional growth rate is positive, the point $x_{ \pm}=0$ is located in the lower (respectively upper) half complex $r$-plane (since $\Omega_{c}^{\prime}<0$ ) at a distance $O(|\epsilon|)$ from the real $r$-axis. Thus, we have $\left|\Lambda x_{ \pm}\right| \gg 1$ with $\left|\arg \left( \pm \mathrm{i} \Lambda x_{ \pm}\right)\right|>\pi / 3$ along the real $r$-axis so that the Scorer's function in (3.28) can be approximated by $\mathrm{Hi}\left( \pm \mathrm{i} \Lambda x_{ \pm}\right) \sim \mathrm{i} /\left( \pm \pi \Lambda x_{ \pm}\right)$. This implies that the typical amplitude of $W_{ \pm}$then scales as $1 /|\epsilon|$ and the typical width of the critical layer is $|\epsilon|$.

A composite approximation uniformly valid in $r$ can be constructed from (3.27) and (3.28):

$$
W_{ \pm}=W^{i}+W_{ \pm}^{v}+\Omega_{c} r_{c} \frac{\Omega_{c}+R o^{(l)^{-1}}}{2 F_{h}^{(l)^{2}} \Omega_{c}^{\prime}\left(r-r_{c}\right)} \text {. }
$$


In the following, we shall use this composite approximation when a critical point exists.

\subsubsection{Order- $\epsilon$ problem}

At order $\epsilon$, the horizontal momentum equations can be written in a compact and convenient form in terms of an equation for the the first-order vertical vorticity $\tilde{\zeta}_{1}$ :

$$
\Omega \frac{\partial \tilde{\zeta}_{1}}{\partial \theta}+\tilde{u}_{r 1} \zeta^{\prime}=-\underbrace{\frac{\partial \tilde{\zeta}_{0}}{\partial T}}_{(a)}-\underbrace{\boldsymbol{U}_{s} \cdot \nabla \tilde{\zeta}_{0}-\tilde{\boldsymbol{u}}_{h 0} \cdot \nabla \Delta \psi_{s}}_{(b)}+\underbrace{\mathrm{i} \frac{k^{2}}{\epsilon}\left(\frac{1}{R o^{(l)}}+\zeta\right) \tilde{u}_{z 0}}_{(c)},
$$

and the divergence equation is

$$
\frac{1}{r} \frac{\partial r \tilde{u}_{r 1}}{\partial r}+\frac{1}{r} \frac{\partial \tilde{u}_{\theta 1}}{\partial \theta}+\mathrm{i} \frac{k^{2}}{\epsilon} \tilde{u}_{z 0}=0 .
$$

As seen in (3.31), the first-order vertical-vorticity perturbation $\tilde{\zeta}_{1}$ is forced by three different terms. The term $(a)$ corresponds to the evolution of the zeroth-order vertical-vorticity perturbation on the slow time scale $T$. The term $(b)$ describes the advection of the zeroth-order vertical-vorticity perturbation $\tilde{\zeta}_{0}$ by the straining flow $\boldsymbol{U}_{s}$ and, conversely, the advection of the vertical vorticity $\Delta \psi_{s}$ of the straining flow by the zeroth-order velocity perturbation $\tilde{\boldsymbol{u}}_{h 0}$. The last term $(c)$ represents the stretching of the vertical vorticity of the basic flow by the zeroth-order vertical-velocity perturbation $\tilde{u}_{z 0}$. The latter vertical velocity also appears as a forcing in the divergence equation (3.32). These terms correspond to the leading three-dimensional effects and are included at this order due to the assumption $\mu^{2}=O(\epsilon)$.

Note that the viscous diffusion of $\tilde{\zeta}_{1}$ is always negligible at leading order in Reynolds number even when a critical point $r_{c}$ exists. Therefore, the viscous effects do not need to be considered in (3.31) and are present only implicitly through the vertical velocity $\tilde{u}_{z 0}$ when there is a critical point.

In order to solve (3.31) and (3.32), we decompose the horizontal velocity into rotational and potential components with a streamfunction $\tilde{\psi}_{1}$ and a potential $\tilde{\Phi}_{1}$ :

$$
\tilde{\boldsymbol{u}}_{h 1}=-\nabla \times\left(\tilde{\psi}_{1} \boldsymbol{e}_{z}\right)+\nabla_{h} \tilde{\Phi}_{1}
$$

Using (3.26), the divergence equation (3.32) becomes

$$
\Delta_{h} \tilde{\Phi}_{1}=-\mathrm{i} \frac{k^{2}}{\epsilon}\left(W_{+} C_{+}^{(l)} \mathrm{e}^{\mathrm{i} \theta}-W_{-} C_{-}^{(l)} \mathrm{e}^{-\mathrm{i} \theta}\right) .
$$

The solution is found by reduction of order to be

$$
\tilde{\Phi}_{1}=-\mathrm{i} \frac{k^{2}}{\epsilon}\left(\mathscr{H}_{+}(r) C_{+}^{(l)} \mathrm{e}^{\mathrm{i} \theta}-\mathscr{H}_{-}(r) C_{-}^{(l)} \mathrm{e}^{-\mathrm{i} \theta}\right),
$$

with

$$
\mathscr{H}_{ \pm}(r)=\frac{r}{2} \int_{\infty}^{r} W_{ \pm}(\eta) \mathrm{d} \eta-\frac{1}{2 r} \int_{0}^{r} \eta^{2} W_{ \pm}(\eta) \mathrm{d} \eta,
$$

where the limits of integration have been chosen so that $\tilde{\Phi}_{1}$ is not singular at $r=0$ and vanishes as $r \rightarrow \infty$ for finite Froude number $F_{h}$.

The functions $W_{ \pm}$in (3.36) are given by the inviscid expression (3.27) when $F_{h}^{(l)}<1 / \Omega_{\max }$ and by the composite approximation (3.30) otherwise. However, as usual for viscous critical layers (Lin 1955; Drazin \& Reid 1981; Huerre \& Rossi 1998; Le Dizès 2004), the effect of the critical layer can be more simply taken 
into account by using the inviscid function (3.27) for all radius but by bypassing the singularity in the complex plane. This equivalence is based on the fact that the integral of Scorer's function verifies $\int^{x} \operatorname{Hi}(\xi) \mathrm{d} \xi \sim-\ln x / \pi$ for $x \rightarrow \infty$ with $|\arg (x)|>\pi / 3$. Thus, when integrating, for example, the viscous function $W_{+}^{v}$ given by (3.28) from one side of the critical point to the other side, we have

$$
\int_{r_{c}-\delta r}^{r_{c}+\delta r} W_{+}^{v} \mathrm{~d} r \sim-\mathrm{i} \Omega_{c} r_{c} \pi \frac{\Omega_{c}+R o^{(l)}-1}{2 F_{h}^{(l)^{2}} \Omega_{c}^{\prime}} \operatorname{sgn}\left(\Gamma^{(l)} \Omega_{c}^{\prime}\right),
$$

where $\delta r$ is much larger than the typical width of the critical layer. The same result is obtained by integrating the inviscid function $W_{+}$along a path, avoiding the singularity in the upper (respectively lower) half complex plane when $\operatorname{sgn}\left(\Gamma^{(l)} \Omega_{c}^{\prime}\right)$ is negative (respectively positive). Since $\Omega_{c}^{\prime}<0$ for most angular velocity profiles, the following rule should be adopted when computing the function with subscript + in (3.36):

the contour of integration has to be deformed in the upper half complex plane when the dimensional circulation of the vortex is positive: $\Gamma^{(l)}>0$ and in the lower half when $\Gamma^{(l)}<0$.

The rule is reversed for the function with subscript - .

An example of this equivalence is illustrated in figure $2(b)$, which shows the first integral of (3.36), i.e. $\int_{\infty}^{r} W_{+}(\eta) \mathrm{d} \eta$. The bold lines represent the result of the integration of the composite approximation (3.30) while the thin lines are the result of the integration of the inviscid solution (3.27) in the complex plane. We see that both methods give the same result outside the critical layer and lead, in particular, to the same phase jump when crossing $r_{c}$. However, it should be stressed that the amplitudes of the variations inside the critical layer are arbitrary for the contour-deformation method and depends directly on the radius of the small semicircular detour used to avoid the singularity.

By introducing the decomposition (3.33) in (3.31), we obtain an equation for the streamfunction $\widetilde{\psi}_{1}$ :

$$
\Omega \frac{\partial \Delta_{h} \tilde{\psi}_{1}}{\partial \theta}-\frac{1}{r} \frac{\partial \tilde{\psi}_{1}}{\partial \theta} \zeta^{\prime}=\underbrace{-\boldsymbol{U}_{s} \cdot \nabla \tilde{\zeta}_{0}-\tilde{\boldsymbol{u}}_{h 0} \cdot \nabla \Delta \psi_{s}}_{(b)}-\frac{\partial \tilde{\zeta}_{0}}{\partial T}+\mathrm{i} \frac{k^{2}}{\epsilon}\left(\frac{1}{R o^{(l)}}+\zeta\right) \tilde{u}_{z 0}-\frac{\partial \tilde{\Phi}_{1}}{\partial r} \zeta^{\prime} .
$$

Using (3.19) and (3.24), the straining terms, denoted by (b) in (3.39), can be rewritten as

$$
(b)=J\left(\psi_{s}, \Delta x^{(l)} \frac{\partial \Delta \psi_{a}^{(l)}}{\partial x}+\Delta y^{(l)} \frac{\partial \Delta \psi_{a}^{(l)}}{\partial y}\right)+J\left(\Delta x^{(l)} \frac{\partial \psi_{a}^{(l)}}{\partial x}+\Delta y^{(l)} \frac{\partial \psi_{a}^{(l)}}{\partial y}, \Delta \psi_{s}\right) .
$$

From (2.9), the streamfunction of the straining flow $\psi_{s}$ satisfies

$$
J\left(\psi_{s}, \Delta \psi_{a}^{(l)}\right)+J\left(\psi_{a}^{(l)}, \Delta \psi_{s}\right)=0 .
$$

By deriving this equation with respect to $x$ and $y$, we find that

$$
(b)=\Omega \frac{\partial \Delta_{h} \tilde{\psi}_{1 s}}{\partial \theta}-\frac{1}{r} \frac{\partial \tilde{\psi}_{1 s}}{\partial \theta} \zeta^{\prime} \quad \text { with } \quad \tilde{\psi}_{1 s}=-\Delta x^{(l)} \frac{\partial \psi_{s}}{\partial x}-\Delta y^{(l)} \frac{\partial \psi_{s}}{\partial y} .
$$

Therefore, the solution forced by the straining terms in (3.39) is $\tilde{\psi}_{1 s}$ and simply corresponds to a displacement by an amount equal to $\left(\Delta x^{(l)}, \Delta y^{(l)}\right)$ of the straining flow 
$\psi_{s}$ like the leading-order perturbation (3.20). This means that the total perturbation $\tilde{\psi}_{0}+\epsilon \tilde{\psi}_{1 s}$ consists of a displacement of the elliptic vortex as a whole. Using (2.13) and the relations $(3.21)-(3.22)$, the streamfunction $\tilde{\psi}_{1 s}$ can be explicitly written as

$$
\begin{aligned}
\tilde{\psi}_{1 s}=-\tilde{f} r\left(C_{+}^{(l)} \mathrm{e}^{\mathrm{i} \theta}+C_{-}^{(l)} \mathrm{e}^{-\mathrm{i} \theta}\right)+ & \left(\frac{h^{\prime}(r)}{4}+\frac{h(r)}{2 r}-r\right)\left(C_{-}^{(l)} \mathrm{e}^{\mathrm{i} \theta}+C_{+}^{(l)} \mathrm{e}^{-\mathrm{i} \theta}\right) \\
& +\left(\frac{h^{\prime}(r)}{4}-\frac{h(r)}{2 r}\right)\left(C_{+}^{(l)} \mathrm{e}^{3 \mathrm{i} \theta}+C_{-}^{(l)} \mathrm{e}^{-3 \mathrm{i} \theta}\right) .
\end{aligned}
$$

The last two terms of the right-hand side of (3.39) correspond to three-dimensional effects. The corresponding solution can be obtained by reduction of order. Therefore, the whole solution of (3.39) can be found analytically:

$$
\begin{aligned}
\tilde{\psi}_{1}=\tilde{\psi}_{1 s}-\mathrm{i} r\left(\frac{\partial C_{+}^{(l)}}{\partial T} \mathrm{e}^{\mathrm{i} \theta}-\frac{\partial C_{-}^{(l)}}{\partial T} \mathrm{e}^{-\mathrm{i} \theta}\right) \\
+\frac{k^{2}}{\epsilon}\left[\left(\mathscr{F}_{+}(r)-r \mathscr{H}_{+}^{\prime}(r)\right) C_{+}^{(l)} \mathrm{e}^{\mathrm{i} \theta}+\left(\mathscr{F}_{-}(r)-r \mathscr{H}_{-}^{\prime}(r)\right) C_{-}^{(l)} \mathrm{e}^{-\mathrm{i} \theta}\right],
\end{aligned}
$$

where

$$
\mathscr{F}_{ \pm}(r)=r \Omega(r)\left[\int_{0}^{r} \frac{W_{ \pm}(\eta)}{\Omega(\eta)} \mathrm{d} \eta+\int_{0}^{r} \frac{\mathrm{d} \eta}{\eta^{3} \Omega^{2}(\eta)} \int_{0}^{\eta}\left(\Omega(\xi)+\frac{1}{R o^{(l)}}\right) \xi^{2} W_{ \pm}(\xi) \mathrm{d} \xi\right] .
$$

As before, the inviscid expression (3.27) of $W_{ \pm}$can be used for all $r$ when $F_{h}^{(l)}>1 / \Omega_{\max }$ provided that the contour of integration is deformed in the complex plane around the singularity $r_{c}$ according to the rule (3.38) for the function with subscript + and the reversed rule for the function with subscript - .

The first-order solution generally behaves like $\tilde{\psi}_{1} \propto r$ for large $r$. Note that there is also a term proportional to $r \ln r$ in $\mathscr{F}_{ \pm}(r)$, as shown in Appendix B but this does not substantially modify the present reasoning. Since the leading-order inner solution $\tilde{\psi}_{0}$ behaves as $1 / r$ for large $r$, this implies that the inner solution $\tilde{\psi}=\tilde{\psi}_{0}+\epsilon \tilde{\psi}_{1}+\cdots$ is not uniformly asymptotic for all $r$ but valid only close to the core of the left vortex such that $r \ll \min (\tilde{b}, 1 / \mu)$. In the latter inequality, the two parameters $\tilde{b}$ and $1 / \mu$ are considered separately even if they are assumed to be formally of the same magnitude. The purpose is to encompass the case of a single vortex for which $\tilde{b} \rightarrow \infty$ but $\mu$ is non-zero. The goal of the next subsection is to find a solution valid for large radius.

\subsection{Outer region}

We now assume that we are far from the cores of the two vortices such that $r=O(d)$, where $d$ is an arbitrary large distance: $d \gg 1$. By introducing a long-range variable $u=r / d$, the basic flow outside the vortex cores given by (2.14) becomes

$$
\begin{aligned}
U_{b r} & =-\frac{\tilde{\Gamma}}{\tilde{b}}\left(\frac{\tilde{b}^{2}}{d^{2} u^{2}-2 d \tilde{b} u \cos \theta+\tilde{b}^{2}}-1\right) \sin \theta, \\
U_{b \theta} & =\frac{1}{d u}+\tilde{\Gamma}\left(\frac{d u-\tilde{b} \cos \theta}{d^{2} u^{2}-2 d \tilde{b} u \cos \theta+\tilde{b}^{2}}\right)+\frac{\tilde{\Gamma}}{\tilde{b}} \cos \theta-\tilde{f} \epsilon d u .
\end{aligned}
$$

The region $u \approx \tilde{b} / d$ and $\theta \approx 0$ is excluded since it corresponds to the inner region of the right vortex where the perturbation has to be calculated in the same way as in the previous subsection. We see that the order of magnitude of the base flow for $u=O(1)$ 
is $\boldsymbol{U}_{b}=O\left(d \epsilon_{U}\right)$ where

$$
\epsilon_{U}=\max \left(|\epsilon|, 1 / d^{2}\right)
$$

is a small parameter.

In order to find the solution in this region, it is convenient to rescale the linearized governing equations (3.2)-(3.5) with the long-range radius $u$ :

$$
\begin{gathered}
\epsilon \frac{\partial \tilde{\boldsymbol{u}}_{h}}{\partial T}+\epsilon_{U}\left(\overline{\boldsymbol{U}}_{b} \cdot \bar{\nabla}_{h} \tilde{\boldsymbol{u}}_{h}+\tilde{\boldsymbol{u}}_{h} \cdot \bar{\nabla}_{h} \overline{\boldsymbol{U}}_{b}\right)+\left(2 \tilde{f} \epsilon+\frac{1}{R o^{(l)}}\right) \boldsymbol{e}_{z} \times \tilde{\boldsymbol{u}}_{h}=-\bar{\nabla}_{h} \tilde{\Pi}, \\
F_{h}^{(l)^{2}}\left(\epsilon \frac{\partial \tilde{u}_{z}}{\partial T}+\epsilon_{U} \overline{\boldsymbol{U}}_{b} \cdot \bar{\nabla}_{h} \tilde{u}_{z}\right)=-\mathrm{i} k d \tilde{\Pi}-\tilde{\rho} \\
\bar{\nabla}_{h} \cdot \tilde{\boldsymbol{u}}_{h}+\mathrm{i} k d \tilde{u}_{z}=0, \\
\epsilon \frac{\partial \tilde{\rho}}{\partial T}+\epsilon_{U} \overline{\boldsymbol{U}}_{b} \cdot \bar{\nabla}_{h} \tilde{\rho}=\tilde{u}_{z},
\end{gathered}
$$

where the basic flow and the pressure have been rescaled: $\overline{\boldsymbol{U}}_{b}=\boldsymbol{U}_{b} /\left(d \epsilon_{U}\right)=O(1)$, $\tilde{\Pi}=\tilde{p} / d$ and the horizontal gradient is with respect to the stretched coordinates: $\bar{\nabla}_{h}=d \nabla_{h}$. The equation for the vertical vorticity $\tilde{\zeta}=\left(\bar{\nabla} \times \tilde{\boldsymbol{u}}_{h}\right) \boldsymbol{e}_{z}$ becomes

$$
\epsilon \frac{\partial \tilde{\zeta}}{\partial T}+\epsilon_{U} \overline{\boldsymbol{U}}_{b} \cdot \bar{\nabla}_{h} \tilde{\zeta}-\mathrm{i} \frac{k d}{R o^{(l)}} \tilde{u}_{z}=0,
$$

where we have used the fact that the vertical vorticity of the basic flow (3.46)-(3.47) is uniform: $\left(\nabla \times \boldsymbol{U}_{b}\right) \boldsymbol{e}_{z}=-2 \tilde{f} \epsilon$. Using (3.52), (3.53) leads to the equation for the conservation of potential vorticity:

$$
\epsilon \frac{\partial \tilde{q}}{\partial T}+\epsilon_{U} \overline{\boldsymbol{U}}_{b} \cdot \bar{\nabla}_{h} \tilde{q}=0, \quad \text { where } \quad \tilde{q}=\tilde{\zeta}-\mathrm{i} \frac{k d}{R o^{(l)}} \tilde{\rho} .
$$

Since the potential vorticity of the perturbation is initially zero outside the vortex cores and is advected by the base flow like a passive tracer, it is legitimate to assume that it will remain zero for all time: $\tilde{q}=0$, i.e.

$$
\tilde{\zeta}=\mathrm{i} \frac{k d}{R o^{(l)}} \tilde{\rho}
$$

We also note that (3.52) implies that the vertical velocity $\tilde{u}_{z}$ is one order of magnitude in $\epsilon_{U}$ smaller than $\tilde{\rho}$. Thus, (3.50) reduces to the hydrostatic balance when $F_{h}^{(l)} \epsilon_{U} \ll 1$ :

$$
-\mathrm{i} k d \tilde{\Pi}-\tilde{\rho}=0+O\left(\left(F_{h}^{(l)} \epsilon_{U}\right)^{2}\right) .
$$

The parameter $k d$ appearing in (3.56) and (3.51) will be assumed arbitrary since $k$ is small but $d$ is large. In this case, the outer perturbation can be expanded with the small parameter $\epsilon_{U}$ :

$$
\begin{aligned}
\tilde{\boldsymbol{u}}_{h} & =\tilde{\boldsymbol{u}}_{h 0}+\epsilon_{U} \tilde{\boldsymbol{u}}_{h 1}+\cdots, \\
\tilde{\Pi} & =\tilde{\Pi}_{0}+\epsilon_{U} \tilde{\Pi}_{1}+\cdots, \\
\tilde{u}_{z} & =\epsilon_{U} \tilde{u}_{z 1}+\cdots, \\
\tilde{\rho} & =\tilde{\rho}_{0}+\epsilon_{U} \tilde{\rho}_{1}+\cdots .
\end{aligned}
$$

Two different cases need to be considered according to the magnitude of the Rossby number $R o^{(l)}: R o^{(l)} \ll O\left(1 / \epsilon_{U}\right)$ and $R o^{(l)} \geqslant O\left(1 / \epsilon_{U}\right)$. 


\subsubsection{Outer solution for $R o^{(l)} \ll O\left(1 / \epsilon_{U}\right)$}

Inserting the asymptotic series (3.57)-(3.60) in (3.49)-(3.51) yields at leading order

$$
\begin{gathered}
\frac{1}{R o^{(l)}} \boldsymbol{e}_{z} \times \tilde{\boldsymbol{u}}_{h 0}=-\bar{\nabla}_{h} \tilde{\Pi}_{0}, \\
-\mathrm{i} k d \tilde{\Pi}_{0}-\tilde{\rho}_{0}=0, \\
\bar{\nabla}_{h} \cdot \tilde{\boldsymbol{u}}_{h 0}=0,
\end{gathered}
$$

while $(3.55)$ is

$$
\tilde{\zeta}_{0}=\mathrm{i} \frac{k d}{R o^{(l)}} \tilde{\rho}_{0} .
$$

The horizontal velocity at leading order is therefore given by

$$
\tilde{\boldsymbol{u}}_{h 0}=-\bar{\nabla} \times\left(\tilde{\psi}_{0} / d \boldsymbol{e}_{z}\right)+\bar{\nabla}_{h} \tilde{\Phi}_{0} / d
$$

with

$$
\bar{\Delta}_{h} \tilde{\Phi}_{0}=0 \text { and } \bar{\Delta}_{h} \tilde{\psi}_{0}=\left(\frac{k d}{R o^{(l)}}\right)^{2} \tilde{\psi}_{0} .
$$

These equations are those of a quasi-geostrophic flow. This is not surprising since we are far from the two vortex cores where the base flow (3.46)-(3.47) is small. Thus, the local Rossby number $R o^{(l)} \epsilon_{U}$ and the Froude number $F_{h}^{(l)} \epsilon_{U}$ based on the magnitude of the base flow in this region are both small even if the Rossby number $R o^{(l)}$ and the Froude number $F_{h}^{(l)}$ based on the characteristics of the vortex core are large.

The solutions of (3.66b) which decay at infinity are of the form $K_{m}(\beta d u) \mathrm{e}^{ \pm \mathrm{i} m \theta}$, i.e. expressed in terms of the unstretched radius: $K_{m}(\beta r) \mathrm{e}^{ \pm \mathrm{i} m \theta}$, where $K_{m}$ is the modified Bessel function of the second kind of order $m$ and $\beta=k /\left|R o^{(l)}\right|=2 \hat{k} R^{(l)}\left|\Omega_{b}\right| / N$. The solutions of (3.66a) which decay at infinity are simply of the form $r^{-m} \mathrm{e}^{ \pm \mathrm{i} m \theta}$. In order to be consistent with the inner solution of each vortex, the streamfunction is taken as the superposition of two solutions with azimuthal wavenumbers $|m|=1$ : one centred on the left vortex and the other centred on the right vortex:

$$
\tilde{\psi}_{0}=\beta\left[K_{1}(\beta r)\left(E_{+}^{(l)} e^{\mathrm{i} \theta}+E_{-}^{(l)} \mathrm{e}^{-\mathrm{i} \theta}\right)+\tilde{\Gamma} K_{1}(\beta \xi)\left(E_{+}^{(r)} \mathrm{e}^{\mathrm{i} \eta}+E_{-}^{(r)} \mathrm{e}^{-\mathrm{i} \eta}\right)\right],
$$

where $(\xi, \eta)$ are the cylindrical coordinates centred on the right vortex (figure 1) and $E_{ \pm}^{(l)}, E_{ \pm}^{(r)}$ are constants that will be later related to the displacements of each vortex due to the matching between the inner and outer solutions. The additional factors $\beta$ and $\tilde{\Gamma}$ have been included in (3.67) in order to simplify the matching procedure. Note that the potential $\tilde{\Phi}_{0}$ should also be chosen so as to match the $1 / r$ behaviour of the inner potential $\tilde{\Phi}_{1}$ for large $r$.

\subsubsection{Outer solution for $\operatorname{Ro}^{(l)} \geqslant O\left(1 / \epsilon_{U}\right)$}

In this case, the leading-order equations are identical to the previous ones except that the horizontal momentum equation (3.61) becomes

$$
0=-\bar{\nabla}_{h} \tilde{\Pi}_{0}
$$

Thus, we have $\tilde{\Pi}_{0}=0$ leading to

$$
\bar{\Delta}_{h} \tilde{\Phi}_{0}=0 \quad \text { and } \quad \bar{\Delta}_{h} \tilde{\psi}_{0}=0 .
$$

Equations (3.69) are those of a two-dimensional potential flow. The solution which decays at infinity and will match the inner solutions of each vortex can be written in 
the form

$$
\tilde{\psi}_{0}=\frac{1}{r}\left(E_{+}^{(l)} \mathrm{e}^{\mathrm{i} \theta}+E_{-}^{(l)} \mathrm{e}^{-\mathrm{i} \theta}\right)+\frac{\tilde{\Gamma}}{\xi}\left(E_{+}^{(r)} \mathrm{e}^{\mathrm{i} \eta}+E_{-}^{(r)} \mathrm{e}^{-\mathrm{i} \eta}\right) .
$$

We can notice that setting $1 / R o^{(l)} \leqslant O\left(\epsilon_{U}\right)$ in (3.67) also gives the same solution at the order considered herein. It is therefore correct to use (3.67) for all the values of the Rossby number.

In contrast, solution (3.67) is restricted to the regime of small and moderate Froude number: $F_{h}^{(l)} \ll 1 / \epsilon_{U}$ in order that the hydrostatic balance (3.56) is satisfied. This implies that the following three conditions should hold:

$$
F_{h}^{(l)} \ll \frac{1}{|\epsilon|}, \quad F_{h}^{(l)} \ll \frac{1}{\mu^{2}}, \quad F_{h}^{(l)} \ll r^{2} .
$$

In dimensional form, the first condition (i.e. (3.71a)) is $\Gamma^{(r)} /\left(2 \pi b^{2}\right) \ll N$, i.e. the dimensional strain exerted by the companion vortex should be much smaller than the Brunt-Väisäilä frequency. Since the strain is typically small, this condition is fulfilled over a wide range of Froude number. Condition (3.71b) is equivalent to (3.71a) when $\mu^{2}=O(\epsilon)$. However, when the separation distance is very large, $\tilde{b} \gg 1 / \mu$, and, in particular, in the case of a single vortex (i.e. $\tilde{b}=\infty)$, $(3.71 b)$, together with the assumption (3.7), implies that the solution (3.67) is valid only in the wavenumber range $0 \leqslant k \ll \min \left(1,\left|R o^{(l)}\right|\right) \max \left(1, \sqrt{F_{h}^{(l)}}\right)$. Finally, the last condition (i.e. (3.71c)), combined with the assumption $r \gg 1$ made at the beginning of $\S 3.2$, shows that (3.67) is valid only for large radius such that $r \gg \max \left(\sqrt{F_{h}^{(l)}}, 1\right)$.

\subsection{Matching}

The inner solution is valid for $r \ll \min (\tilde{b}, 1 / \mu)$ while the outer solution (3.67) is valid for $r \gg \max \left(\sqrt{F_{h}^{(l)}}, 1\right)$. Therefore, these two solutions should match in the overlap region:

$$
\max \left(\sqrt{F_{h}^{(l)}}, 1\right) \ll r \ll \min (\tilde{b}, 1 / \mu) .
$$

Such range exists due to the assumptions $(3.71 a, b)$ and (3.7). We first express the outer solution (3.67) in this overlap region. The cylindrical coordinates $(\xi, \eta)$ appearing in (3.67) can be expressed in terms of $(r, \theta)$ using the relations $r \cos \theta-\tilde{b}=\xi \cos \eta$ and $r \sin \theta=\xi \sin \eta$. Since $r \ll \tilde{b}$ in the overlap region, we have

$$
\xi=\tilde{b}\left[1-r \tilde{b}^{-1} \cos \theta+O\left(r^{2} / \tilde{b}^{2}\right)\right] \text { and } \mathrm{e}^{\mathrm{i} \eta}=-1+\mathrm{i} r \tilde{b}^{-1} \sin \theta+O\left(r^{2} / \tilde{b}^{2}\right) .
$$

Therefore, the outer solution (3.67) at leading orders becomes

$$
\begin{aligned}
\tilde{\psi}_{\text {out }}= & {\left[\frac{1}{r}+\frac{\beta^{2} r}{2}\left(\ln \left(\frac{\beta r}{2}\right)-\frac{1}{2}+\gamma_{e}\right)\right]\left(E_{+}^{(l)} \mathrm{e}^{\mathrm{i} \theta}+E_{-}^{(l)} \mathrm{e}^{-\mathrm{i} \theta}\right) } \\
& +\frac{\epsilon r}{2}\left[\Psi\left(E_{+}^{(r)}-E_{-}^{(r)}\right)\left(\mathrm{e}^{\mathrm{i} \theta}-\mathrm{e}^{-\mathrm{i} \theta}\right)-\chi\left(E_{+}^{(r)}+E_{-}^{(r)}\right)\left(\mathrm{e}^{\mathrm{i} \theta}+\mathrm{e}^{-\mathrm{i} \theta}\right)\right] \\
& +O\left(\beta^{4} r^{3} \ln (\beta r), r^{2} / \tilde{b}^{3}, \epsilon / r\right),
\end{aligned}
$$

up to an arbitrary constant and where $\gamma_{e}=0.5772 \ldots$ is Euler's constant. The functions $\chi$ and $\Psi$ are given by

$$
\chi(\hat{\beta} b)=\hat{\beta} b K_{1}(\hat{\beta} b)+\hat{\beta}^{2} b^{2} K_{0}(\hat{\beta} b), \quad \Psi(\hat{\beta} b)=\hat{\beta} b K_{1}(\hat{\beta} b),
$$

where

$$
\hat{\beta}=\beta / R^{(l)}=2 \hat{k}\left|\Omega_{b}\right| / N
$$


is a rescaled dimensional vertical wavenumber independent of the characteristics of each vortex. These two functions will be later seen to be the equivalent in stratified and rotating fluids of the first and second mutual-induction functions of Crow (1970) in homogeneous fluids. The function $\Psi$ and $\chi$ simply describe an advection of the left vortex along the $x$ and $y$ axes, respectively, by the horizontal velocity of the disturbance of the right vortex.

Note that we shall consider that the terms $O\left(\beta^{2} \ln \beta\right)$ in (3.74) are formally of the same order as those $O\left(\beta^{2}\right)$ in order to avoid the definition of an additional slow timescale $O\left(\beta^{2} \ln \beta\right)$. This simplifies the presentation and does not detract from the rigour of the analysis as long as higher-order terms are not computed.

The main difficulty in obtaining the expression of the inner solution in the overlap region is determining the behaviour of the functions $\widetilde{F}_{ \pm}(r)$ for $r \gg \max \left(\sqrt{F_{h}^{(l)}}, 1\right)$. This analysis is carried out in Appendix B. From the final result (B 10), the expression of the inner solution $\tilde{\psi}_{i n}=\tilde{\psi}_{0}+\epsilon \tilde{\psi}_{1}+O\left(\epsilon^{2}\right)$ can be obtained easily, where $\tilde{\psi}_{0}$ is given by (3.19) and $\tilde{\psi}_{1}$ by (3.44):

$$
\begin{aligned}
\tilde{\psi}_{i n}= & \frac{1}{r}\left(C_{+}^{(l)} \mathrm{e}^{\mathrm{i} \theta}+C_{-}^{(l)} \mathrm{e}^{-\mathrm{i} \theta}\right)-\epsilon r\left[C_{-}^{(l)} \mathrm{e}^{\mathrm{i} \theta}+C_{+}^{(l)} \mathrm{e}^{-\mathrm{i} \theta}+\mathrm{i} \frac{\partial C_{+}^{(l)}}{\partial T} \mathrm{e}^{\mathrm{i} \theta}-\mathrm{i} \frac{\partial C_{-}^{(l)}}{\partial T} \mathrm{e}^{-\mathrm{i} \theta}\right] \\
& +r\left[\frac{\beta^{2}}{2}\left(\delta\left(F_{h}^{(l)}, R o^{(l)}\right)-\frac{1}{2}+\ln r\right)-\tilde{f} \epsilon\right] C_{+}^{(l)} \mathrm{e}^{\mathrm{i} \theta} \\
& +r\left[\frac{\beta^{2}}{2}\left(\delta^{*}\left(F_{h}^{(l)}, R o^{(l)}\right)-\frac{1}{2}+\ln r\right)-\tilde{f} \epsilon\right] C_{-}^{(l)} \mathrm{e}^{-\mathrm{i} \theta}+O\left(\frac{\epsilon}{r}, \epsilon^{2}\right),
\end{aligned}
$$

where the asterisk denotes the complex conjugate and

$$
\delta\left(F_{h}, R o\right)=\mathscr{D}\left(F_{h}\right)+2 \operatorname{Ro} \mathscr{B}\left(F_{h}\right)+\operatorname{Ro}^{2} \mathscr{A}\left(F_{h}\right)
$$

The parameters $(\mathscr{A}, \mathscr{B}, \mathscr{D})$ are constants depending on the Froude number given by (B 6)-(B 8) in Appendix B. Incidentally, it is worth noting that the function $h$ has disappeared in (3.77) since $h \rightarrow 0$ as $r \rightarrow \infty$. This means that the adaptation of the vortex to the strain (i.e. the elliptic deformation of the vortex) will play no active role in the instability in contrast to the case of the elliptic instability (Moore \& Saffman 1975; Le Dizès \& Laporte 2002). Here, the whole elliptic vortex tube is simply displaced, as demonstrated by (3.20) and (3.42).

There is no difficulty in matching the outer potential $\tilde{\Phi}_{0}$ to the inner potential $\tilde{\Phi}_{1}$ for large $r$ since they both behave like $1 / r$. In contrast, the two streamfunctions (3.74) and (3.77) match only if we impose

$$
E_{+}^{(l)}=C_{+}^{(l)}, \quad E_{-}^{(l)}=C_{-}^{(l)},
$$

and

$$
\begin{aligned}
& \frac{\partial C_{+}^{(l)}}{\partial T}=\mathrm{i} C_{-}^{(l)}+\mathrm{i}\left(\tilde{f}-\frac{\omega^{(l)}}{\epsilon}\right) C_{+}^{(l)}+\frac{\mathrm{i}}{2}\left[\Psi\left(E_{+}^{(r)}-E_{-}^{(r)}\right)-\chi\left(E_{+}^{(r)}+E_{-}^{(r)}\right)\right], \\
& \frac{\partial C_{-}^{(l)}}{\partial T}=-\mathrm{i} C_{+}^{(l)}-\mathrm{i}\left(\tilde{f}-\frac{\omega^{(l) *}}{\epsilon}\right) C_{-}^{(l)}+\frac{\mathrm{i}}{2}\left[\Psi\left(E_{+}^{(r)}-E_{-}^{(r)}\right)+\chi\left(E_{+}^{(r)}+E_{-}^{(r)}\right)\right],
\end{aligned}
$$


which are the evolution equations governing $C_{+}^{(l)}$ and $C_{-}^{(l)}$ over the slow time $T$. The parameter $\omega^{(l)}$ is given by

$$
\omega^{(l)} \equiv \omega\left(\hat{\beta} R^{(l)}, F_{h}^{(l)}, R o^{(l)}\right)=\frac{\left(\hat{\beta} R^{(l)}\right)^{2}}{2}\left[-\ln \left(\frac{\hat{\beta} R^{(l)}}{2}\right)+\delta\left(F_{h}^{(l)}, R o^{(l)}\right)-\gamma_{e}\right] .
$$

The inner perturbation of the right vortex can be determined by means of an analysis identical to the one performed previously. In particular, this analysis leads to relations identical to (3.79) with the superscript $(l)$ replaced by $(r)$ and where the amplitudes $C_{ \pm}^{(r)}$ are related to the displacement perturbations of the right vortex by

$$
\Delta x^{(r)}=-C_{+}^{(r)}-C_{-}^{(r)} \quad \text { and } \quad \Delta y^{(r)}=-\mathrm{i}\left(C_{+}^{(r)}-C_{-}^{(r)}\right) .
$$

We now rewrite (3.80)-(3.81) in terms of these displacement quantities and those of the left vortex defined in (3.21)-(3.22). In addition, we rescale the slow time $T=\epsilon t$ in terms of the dimensional time $\hat{t}=t 2 \pi R^{(l)^{2}} / \Gamma^{(l)}$. This gives

$$
\begin{aligned}
& \frac{\partial \Delta x^{(l)}}{\partial \hat{t}}=-\frac{\Gamma^{(r)}}{2 \pi b^{2}} \Delta y^{(l)}+\frac{\Gamma^{(r)}}{2 \pi b^{2}} \Psi \Delta y^{(r)}+\left(f-\frac{\Gamma^{(l)}}{2 \pi R^{(l)^{2}}} \omega_{r}^{(l)}\right) \Delta y^{(l)}+\frac{\left|\Gamma^{(l)}\right|}{2 \pi R^{(l)^{2}}} \omega_{i}^{(l)} \Delta x^{(l)}, \\
& \frac{\partial \Delta y^{(l)}}{\partial \hat{t}}=-\frac{\Gamma^{(r)}}{2 \pi b^{2}} \Delta x^{(l)}+\frac{\Gamma^{(r)}}{2 \pi b^{2}} \chi \Delta x^{(r)}-\left(f-\frac{\Gamma^{(l)}}{2 \pi R^{(l)^{2}}} \omega_{r}^{(l)}\right) \Delta x^{(l)}+\frac{\left|\Gamma^{(l)}\right|}{2 \pi R^{(l)^{2}}} \omega_{i}^{(l)} \Delta y^{(l)},
\end{aligned}
$$

where $\omega_{r}^{(l)}=\operatorname{Re}\left(\omega^{(l)}\right)$ and $\omega_{i}^{(l)}=\operatorname{Im}\left(\omega^{(l)}\right)$. The corresponding equations for the displacement perturbations of the right vortex have the same form with the superscripts $(r)$ and $(l)$ interchanged

$$
\begin{aligned}
& \frac{\partial \Delta x^{(r)}}{\partial \hat{t}}=-\frac{\Gamma^{(l)}}{2 \pi b^{2}} \Delta y^{(r)}+\frac{\Gamma^{(l)}}{2 \pi b^{2}} \Psi \Delta y^{(l)}+\left(f-\frac{\Gamma^{(r)}}{2 \pi R^{(r)^{2}}} \omega_{r}^{(r)}\right) \Delta y^{(r)}+\frac{\left|\Gamma^{(r)}\right|}{2 \pi R^{(r)^{2}}} \omega_{i}^{(r)} \Delta x^{(r)}, \\
& \frac{\partial \Delta y^{(r)}}{\partial \hat{t}}=-\underbrace{\frac{\Gamma^{(l)}}{2 \pi b^{2}} \Delta x^{(r)}}_{(a)}+\underbrace{\frac{\Gamma^{(l)}}{2 \pi b^{2}} \chi \Delta x^{(l)}}_{(b)}-\underbrace{(f}_{(c)}-\underbrace{\left.\frac{\Gamma^{(r)}}{2 \pi R^{(r)^{2}}} \omega_{r}^{(r)}\right) \Delta x^{(r)}+\frac{\left|\Gamma^{(r)}\right|}{2 \pi R^{(r)^{2}}} \omega_{i}^{(r)} \Delta y^{(r)}}_{(d)},
\end{aligned}
$$

where $\omega^{(r)}=\omega\left(\hat{\beta} R^{(r)}, F_{h}^{(r)}, R o^{(r)}\right)$.

Equations (3.84)-(3.87) have exactly the same form as the stability equations obtained by Crow (1970), Jimenez (1975) and Bristol et al. (2004) for a pair of vortex filaments in homogeneous fluids except that the mutual-induction functions $\Psi$ and $\chi$ and the self-induction function $\omega$ are different. These equations combine the following four different physical effects labelled $(a-d)$ in (3.87):

(a) Strain: This term represents the effect of the strain field from one vortex acting on the bending perturbations of the other vortex. For example, if the right vortex is displaced towards its companion (i.e. $\Delta x^{(r)}<0$ ), it starts to move along the $y$-axis (see (3.87)) since the advection by the left vortex is then slightly larger.

(b) Mutual induction: These terms describe the effects of the bending perturbations of one vortex on its companion. These effects depend on the mutual-induction functions $\Psi(\hat{\beta} b)$ and $\chi(\hat{\beta} b)$, which describe how the perturbation decays outside 
the vortex core and is thus felt by the companion vortex. They are independent of the characteristics of each vortex. Looking again, for example, at (3.87), the term $(b)$ simply shows that if the left vortex is displaced towards the right vortex (i.e. $\Delta x^{(l)}>0$ ), the right vortex starts to move along the $y$-axis since the relative advection by the left vortex is then higher.

(c) This is an orbital rotation effect due to the rotation of the basic vortex pair.

(d) Self-induction: This effect represents the dynamics of each vortex as if it were alone. It makes each sinusoidally bent vortex to rotate rigidly about its own axis at angular velocity $\omega_{r} \Gamma /\left(2 \pi R^{2}\right)$ (we lighten notation by dropping the superscripts $(l)$ or $(r)$ in the following) in the same direction as the flow in the vortex core if $\omega_{r}>0$ and in the opposite direction if $\omega_{r}<0$. When a viscous critical layer is present (i.e. $\omega_{i} \neq 0$ ), the bending deformations decrease in time with the damping rate $\left|\omega_{i} \Gamma\right| /\left(2 \pi R^{2}\right)$. For simplicity, the damping terms have been written in terms of the absolute value of the circulations. This way, the contour of integration near the singularity should always be deformed in the upper complex plane whatever the sign of $\Gamma$ when computing the parameters $\mathscr{A}, \mathscr{B}$ and $\mathscr{D}$.

We recall that these equations are valid for well-separated vortices $b \gg R$ and long vertical wavelength: $\hat{k} F_{h} R \ll \max \left(1, \sqrt{F_{h}}\right) \min (1,|R o|)$ (see $(3.7)$ and $(3.71 b)$ ). The selfinduction and mutual-induction functions derived here for stratified-rotating fluids are valid regardless of the Rossby numbers and when the strains are much smaller than the Brunt-Väisäilä frequency, i.e. $\Gamma /\left(2 \pi b^{2}\right) \ll N$ (see $(3.71 a)$ ). Since $b \gg R$, these conditions are fully satisfied over a wide range of Froude number: $F_{h} \ll b^{2} / R^{2}$.

As shown in Appendix D, viscous and diffusive effects can also be taken into account at leading order in Reynolds number when there is no critical layer, i.e. for $F_{h}<1 / \Omega_{\max }$. In this regime, the self-induction function (3.82) is to be simply replaced by

$$
\omega \rightarrow \omega-\mathrm{i} \frac{\hat{k}^{2} R^{2}}{R e} \mathscr{V},
$$

where $\mathscr{V}$ is a constant defined in (D 22), and it depends on the vortex profile and the numbers $\left(F_{h}, R o, S c\right)$. It is worth emphasizing that this constant is not always positive, i.e. the viscous effects are not always stabilizing.

In the next section, we investigate the behaviours of the self-induction and mutualinduction functions in stratified and rotating inviscid fluids compared to homogeneous fluids. The study of the stability of vortex pairs in stratified and rotating fluids using (3.84)-(3.87) is postponed to Part 2.

\section{Self-induction and mutual-induction functions in stratified and rotating fluids}

The expressions of the mutual-induction and self-induction functions in stratifiedrotating inviscid fluids and homogeneous fluids are summarized in table 1 . Note that the self-induction function $\omega$ is not defined as in Crow (1970). Here, the self-induction function $\omega$ multiplied by $\Gamma /\left(2 \pi R^{2}\right)$ exactly corresponds to the frequency of rotation of the sinusoidally bent vortex whereas the self-induction function $\omega_{c}$ of Crow (1970) is $\omega_{c}=-\omega /\left(\hat{k}^{2} R^{2}\right)$ (note the minus sign).

As seen in table 1 , these functions in stratified-rotating fluids are different from their counterpart in homogeneous fluids. The mutual-induction functions depend on $\hat{\beta} b=2 b \hat{k}\left|\Omega_{b}\right| / N$ in stratified-rotating fluids instead of $\hat{k} b$ in homogeneous fluids. Furthermore, the expressions of $\chi$ and $\Psi$ are inverted. These differences come from 
Function

$$
\begin{array}{cc}
\chi & \hat{\beta} b K_{1}(\hat{\beta} b)+\hat{\beta}^{2} b^{2} K_{0}(\hat{\beta} b) \\
\Psi & \hat{\beta} b K_{1}(\hat{\beta} b) \\
\omega & \frac{\hat{\beta}^{2} R^{2}}{2}\left[-\ln \left(\frac{\hat{\beta} R}{2}\right)+\delta\left(F_{h}, R o\right)-\gamma_{e}\right]
\end{array}
$$

Homogeneous fluids

$$
\begin{gathered}
\hat{k} b K_{1}(\hat{k} b) \\
\hat{k} b K_{1}(\hat{k} b)+\hat{k}^{2} b^{2} K_{0}(\hat{k} b) \\
\frac{\hat{k}^{2} R^{2}}{2}\left(\ln \frac{\hat{k} R}{2}+\gamma_{e}-D\right)
\end{gathered}
$$

TABLE 1. Expressions of the first and second mutual-induction functions $\chi$ and $\Psi$ and self-induction function $\omega$ in stratified-rotating fluids and homogeneous fluids (Crow 1970; Widnall et al. 1971). The parameter $\hat{k}$ is the dimensional wavenumber and $\hat{\beta}=\hat{k} F_{h} /|R o|=2 \hat{k}\left|\Omega_{b}\right| / N$. The parameter $\delta\left(F_{h}, R o\right)$ is defined in (3.78) and $D=\mathscr{D}(0)$ is defined in (B 8). Note that the self-induction function is not defined as in Crow (1970).

the fact that the perturbation outside the vortex core is irrotational in homogeneous fluids whereas the governing equation for the outer perturbation in stratified-rotating fluids states that the potential vorticity is zero (see $(3.66 b)$ ). The mutual-induction functions are equal to unity for $\hat{\beta} b=0$ and decrease exponentially for large $\hat{\beta} b$. They are essentially negligible for $\hat{\beta} b \gtrsim 8$. Note that in the case of a stratified non-rotating fluid $\left(\Omega_{b}=0\right)$, we have $\chi=\Psi=1$ independently of $\hat{k}$ and $b$. The only difference between the displacement equations (3.84)-(3.87) and those for two-dimensional point vortices is then the presence of self-induction effects.

\subsection{General property of the self-induction function}

In order to investigate the properties of the self-induction function in stratifiedrotating fluids, it is convenient to rewrite (3.82) as

$$
\omega=\frac{k^{2}}{2 R o^{2}}\left[-\ln \left(\frac{k}{2|R o|}\right)+\mathscr{D}\left(F_{h}\right)+2 \operatorname{Ro} \mathscr{B}\left(F_{h}\right)+R o^{2} \mathscr{A}\left(F_{h}\right)-\gamma_{e}\right] \text {, }
$$

where we recall that $k=\hat{k} F_{h} R$. Some general results can be first deduced from (4.1) without specifying the vortex profile. When $F_{h}<1 / \Omega_{\max }$, the self-induction function is purely real because the parameters $(\mathscr{A}, \mathscr{B}, \mathscr{D})$ are real. For $R o \neq \infty$, the logarithmic term (first term on the right-hand side of (4.1)) is dominant for sufficiently small $k$, implying that $\omega$ is always positive in this limit. However, the self-induction function $\omega$ is higher for cyclonic vortices $(R o>0)$ than for anticyclonic vortices $(R o<0)$ because the third term on the right-hand side of (4.1) depends on the sign of the Rossby number and $\mathscr{B}$ is positive whatever the vortex profile (see (B 7)). When $R o=\infty$, the self-induction reduces to $\omega=k^{2} \mathscr{A} / 2$. As seen from (B 6), $\mathscr{A}$ is positive for $F_{h}<1 / \Omega_{\max }$ so that the self-induction is also always positive. When $F_{h}>1 / \Omega_{\max }$, the self-induction becomes complex with a negative imaginary part and the sign of the real part of $(\mathscr{A}, \mathscr{B}, \mathscr{D})$ depends on the Froude number.

In summary, the key result is that the self-induction function (4.1) is positive when $F_{h}<1 / \Omega_{\max }$ whatever the vortex profile and the Rossby number. Physically, this means that a sinusoidally curved vortex spins rigidly about its axis in the same direction as the rotation of flow in the vortex core. Strikingly, this is opposite to the case of a homogeneous fluid where the self-induction (as defined herein) is negative for $\hat{k} \ll 1$ :

$$
\omega=\frac{\hat{k}^{2} R^{2}}{2}\left(\gamma_{e}-D-\ln 2+\ln \hat{k} R\right)+\cdots,
$$

where the constant $D$ is the same as $(\mathrm{B} 8)$ for $F_{h}=0$, i.e. $D=\mathscr{D}(0)$. This formula has been obtained by Widnall et al. (1971) and Leibovich et al. (1986) (see also Moore \& 

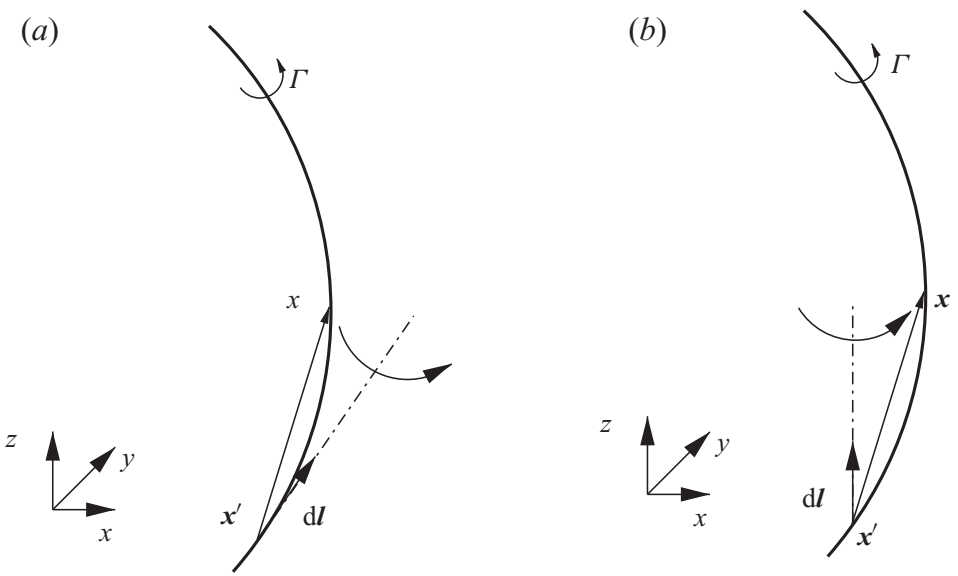

FIGURE 3. Graphical interpretation of the Biot-Savart law in homogeneous fluid $(a)$ and quasi-geostrophic fluid $(b)$. See explanations in text.

Saffman 1972) by means of a long-wavelength matched asymptotic analysis similar to the present one.

The direction of the self-induced motion can be easily understood in the cases of homogeneous fluids and quasi-geostrophic fluids. Indeed, in both cases, the motion induced by a vortex line can be derived from the Biot-Savart law. In homogeneous fluids, the velocity induced at a point $\boldsymbol{x}$ by all the portions $\mathrm{d} \boldsymbol{l}\left(\boldsymbol{x}^{\prime}\right)$ of a vortex filament is

$$
\boldsymbol{u}(\boldsymbol{x})=-\frac{\Gamma}{4 \pi} \int \frac{\left(\boldsymbol{x}-\boldsymbol{x}^{\prime}\right) \times \mathrm{d} \boldsymbol{l}}{\left|\boldsymbol{x}-\boldsymbol{x}^{\prime}\right|^{3}}
$$

It can be shown that the dominant contribution in this integral comes from the portions of the filament in the neighbourhood of $\boldsymbol{x}$ (Batchelor 1967). The cutoff approximation should, however, be used (i.e. the immediate neighbourhood of $\boldsymbol{x}$ should be excluded) to avoid the logarithmic singularity (Widnall et al. 1971). Figure 3( $a$ ) graphically illustrates the velocity induced at point $\boldsymbol{x}$ by a nearby portion of a curved vortex filament. Due to the curvature of the vortex, the point $\boldsymbol{x}$ is located to the left of the line tangent at $\boldsymbol{x}^{\prime}$ (i.e. parallel to $\mathrm{d} \boldsymbol{l}$ ). This directly shows that the induced motion is directed in the negative $y$ direction. The motion induced by the other portions of the filament is also in the same direction. Thus, the self-induced motion tends to rotate the curved vortex in a direction opposite to the flow in the vortex core. law:

In the case of a quasi-geostrophic fluid, the induced motion is given by a similar

$$
\boldsymbol{u}(\tilde{\boldsymbol{x}})=-\frac{\Gamma}{4 \pi} \int \frac{\left(\tilde{\boldsymbol{x}}-\tilde{\boldsymbol{x}}^{\prime}\right) \times \mathrm{d} l \boldsymbol{e}_{z}}{\left|\tilde{\boldsymbol{x}}-\tilde{\boldsymbol{x}}^{\prime}\right|^{3}},
$$

where $\tilde{x}=x \boldsymbol{e}_{x}+y \boldsymbol{e}_{y}+\tilde{z} \boldsymbol{e}_{z}$, where $\tilde{z}=\left(2 \Omega_{b} / N\right) z$ is the rescaled vertical coordinate (see, for example, Miyazaki, Shimada \& Takahashi 2000). The crucial difference is that the vector $\mathrm{d} \boldsymbol{l}=\mathrm{d} \boldsymbol{l} \boldsymbol{e}_{z}$ always remains vertical even when the filament is curved since the motion is constrained to remain horizontal. As sketched in figure $3(b)$, the point $\boldsymbol{x}$ is now located to the right of the vertical line going through $\boldsymbol{x}^{\prime}$ (i.e. parallel to $\mathrm{d} \boldsymbol{l}$ ). Therefore, the induced motion is directed in the positive $y$ direction so that the curved vortex will spin in the same direction as the flow in the vortex core. 
The sign of the self-induction function can also be easily understood in the case of a stratified non-rotating fluid. In this case, we have $\omega=k^{2} \mathscr{A} / 2$ and the parameter $\mathscr{A}$ can be written in terms of the leading-order vertical-velocity amplitude $W_{+}$:

$$
\mathscr{A}=\int_{0}^{\infty} \xi^{2} \Omega(\xi) W_{+}(\xi) \mathrm{d} \xi .
$$

Thus, we see that the real part of $\mathscr{A}$ will be positive when the vertical-velocity amplitude $W_{+}$is positive over all $\xi$. The fact that a positive vertical-velocity amplitude induces a positive self-induced motion is explained in Otheguy et al. (2007) for $F_{h}=0$ (see figure $3 b$ of Otheguy et al. 2007). For example, if we assume a positive displacement of the vortex in the $y$ direction (i.e. $\Delta y>0$ and $\Delta x=0$ ), the divergence equation at leading order is

$$
\nabla \cdot \tilde{\boldsymbol{u}}_{h}=k^{2} \Delta y W_{+} \cos \theta
$$

This shows that the vertical-velocity field generates a divergence for $x=r \cos \theta<0$ and a convergence for $x>0$ when $W_{+}>0$. To satisfy mass conservation, a secondary horizontal motion is thus created in the negative $x$ direction. This tends to rotate the vortex in the same direction as the flow in the vortex core. The vertical-velocity field also stretches and squeezes the basic vorticity so as to displace the vortex in the same direction (see Otheguy et al. 2007).

From the expression of the vertical-velocity amplitude (3.27), we now see that the explanations of Otheguy et al. (2007) are valid not only for $F_{h}=0$ but for all the Froude numbers in the range $F_{h}<1 / \Omega_{\max }$, since the vertical-velocity amplitude $W_{+}$ is then positive for all radius. For $F_{h}>1 / \Omega_{\max }, W_{+}$remains positive for $r>r_{c}$ but becomes negative for $r<r_{c}$. The motions described above are reversed in the latter region. The net effect (i.e. the sign of the real part of $\mathscr{A}$ ) will depend on the relative importance of the two regions. Nevertheless, since the size of the region where $W_{+}$is negative increases with the Froude number, the net self-induced motion is expected to reverse for large Froude number.

\subsection{Self-induction function of the Lamb-Oseen vortex}

In order to provide more quantitative results on the self-induction in stratified-rotating fluids, we now consider the Lamb-Oseen vortex profile

$$
\Omega=\frac{1}{r^{2}}\left(1-\mathrm{e}^{-r^{2}}\right)
$$

The parameters $\mathscr{A}, \mathscr{B}$ and $\mathscr{D}$ corresponding to this profile have been computed numerically and are shown in figure 4 . Note that they can be computed analytically for $F_{h}=0$ :

$$
\left.\begin{array}{l}
\mathscr{A}(0)=9 \ln 3-14 \ln 2=0.1834, \mathscr{B}(0)=\frac{3}{2}(2 \ln 2-\ln 3)=0.4315, \\
\mathscr{D}(0)=\frac{\gamma_{e}-\ln 2}{2}=-0.0579 .
\end{array}\right\}
$$

The value of $\mathscr{A}(0)$ agrees with the asymptotic results obtained by Otheguy et al. (2007) for a strongly stratified non-rotating fluid. As can be seen in figure 4, the parameters $(\mathscr{A}, \mathscr{B}, \mathscr{D})$ are almost constant from $F_{h}=0$ to $F_{h} \approx 0.5$. Their real parts have a peak around $F_{h}=1 / \Omega_{\max }=1$ and for $F_{h}>1$, they have a negative imaginary 

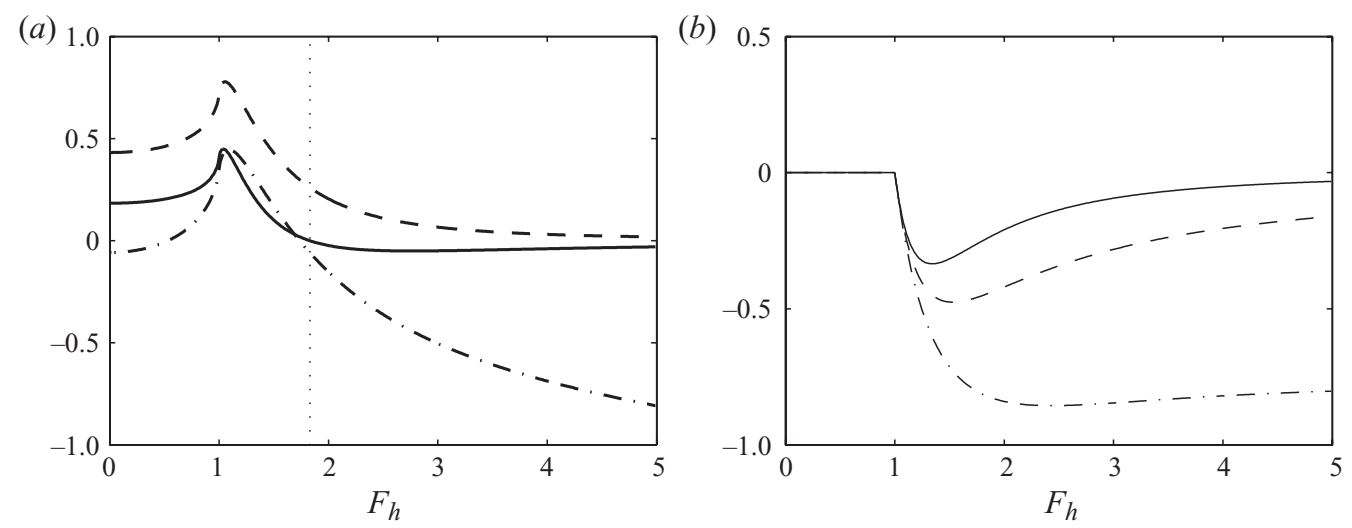

FiguRE 4. Real part $(a)$ and imaginary part $(b)$ of the parameters $\mathscr{A}(-) \mathscr{B}(---)$ and $\mathscr{D}(-\cdot)$ as a function of the Froude number $F_{h}$ for the Lamb-Oseen vortex (4.7). The dotted line in $(a)$ indicates the Froude number $F_{h}=1.83$ for which the real part of $\mathscr{A}$ becomes negative.

part. For large Froude number, they behave like

$$
\left.\begin{array}{l}
\mathscr{A}\left(F_{h}\right)=-\frac{1}{2 F_{h}^{2}}\left[\ln F_{h}+\gamma_{e}-\ln 2+\mathrm{i} \frac{\pi}{2}\right]+O\left(F_{h}^{-4}\right), \\
\mathscr{B}\left(F_{h}\right)=\frac{1}{2 F_{h}^{2}}-\mathrm{i} \frac{\pi}{4 F_{h}}+O\left(F_{h}^{-4}\right), \\
\mathscr{D}\left(F_{h}\right)=-\frac{1}{2} \ln F_{h}-\mathrm{i} \frac{\pi}{4}+O\left(F_{h}^{-4}\right),
\end{array}\right\}
$$

so that $\mathscr{A}$ and $\mathscr{B}$ decrease to zero while $\mathscr{D}$ behaves like the logarithm of the Froude number (figure 4). The approximation (4.9) almost gives the exact values as soon as $F_{h} \gtrsim 4$. The parameters $(\mathscr{A}, \mathscr{B}, \mathscr{D})$ in the simple case of the Rankine vortex have a similar behaviour, as shown in Appendix C.

Figure 5 shows some examples of the self-induction function of the Lamb-Oseen vortex (represented by thin solid lines) for $R o=\infty$ and various Froude numbers from $F_{h}=0.9$ to $F_{h}=10$. We can see that the self-induction has a large negative imaginary part when $F_{h}>1$. It can also be noticed that the real part of the self-induction is positive for $F_{h}=1.67$ and negative for $F_{h}=2$. This is because the sign of the real part of $\mathscr{A}$ changes at $F_{h}=1.83$ (figure 4 ).

These results have been checked by directly computing the frequency of the bending waves of the Lamb-Oseen vortex (4.7) in a stratified-rotating fluid. When there is a single axisymmetric vortex, the perturbations defined in (3.1) can be further written in the form

$$
[\tilde{\boldsymbol{u}}, \tilde{p}, \tilde{\rho}](r, \theta, t)=[\hat{\boldsymbol{u}}, \hat{p}, \hat{\rho}](r) \mathrm{e}^{-\mathrm{i} \omega_{e} t+\mathrm{i} m \theta} .
$$

With our definition of the self-induction function $\omega$, the frequency $\omega_{e}$ of the slow bending wave with azimuthal wavenumber $m=+1$ should be equal to $\omega$ in the limit of small vertical wavenumber.

A single equation for $\varphi=r \hat{u}_{r}$ can be obtained by inserting (4.10) in (3.2)-(3.5):

$$
\left(\frac{\varphi^{\prime}}{r Q}\right)^{\prime}-\left[\frac{m^{2}}{r^{2}}-k^{2} \frac{s^{2}-\phi}{1-F_{h}^{2} s^{2}}+\frac{m}{r^{2} s}\left(r \zeta^{\prime}-\left(\zeta+R o^{-1}\right)\left(\frac{r Q^{\prime}}{Q}+2\right)\right)\right] \frac{\varphi}{r Q}=0
$$

where $s=-\omega_{e}+m \Omega, Q=-k^{2} s^{2} /\left(1-s^{2} F_{h}^{2}\right)+m^{2} / r^{2}$ and $\phi=\left(2 \Omega+R o^{-1}\right)\left(\zeta+R o^{-1}\right)$. This equation has been solved by a shooting method similar to the one described by 

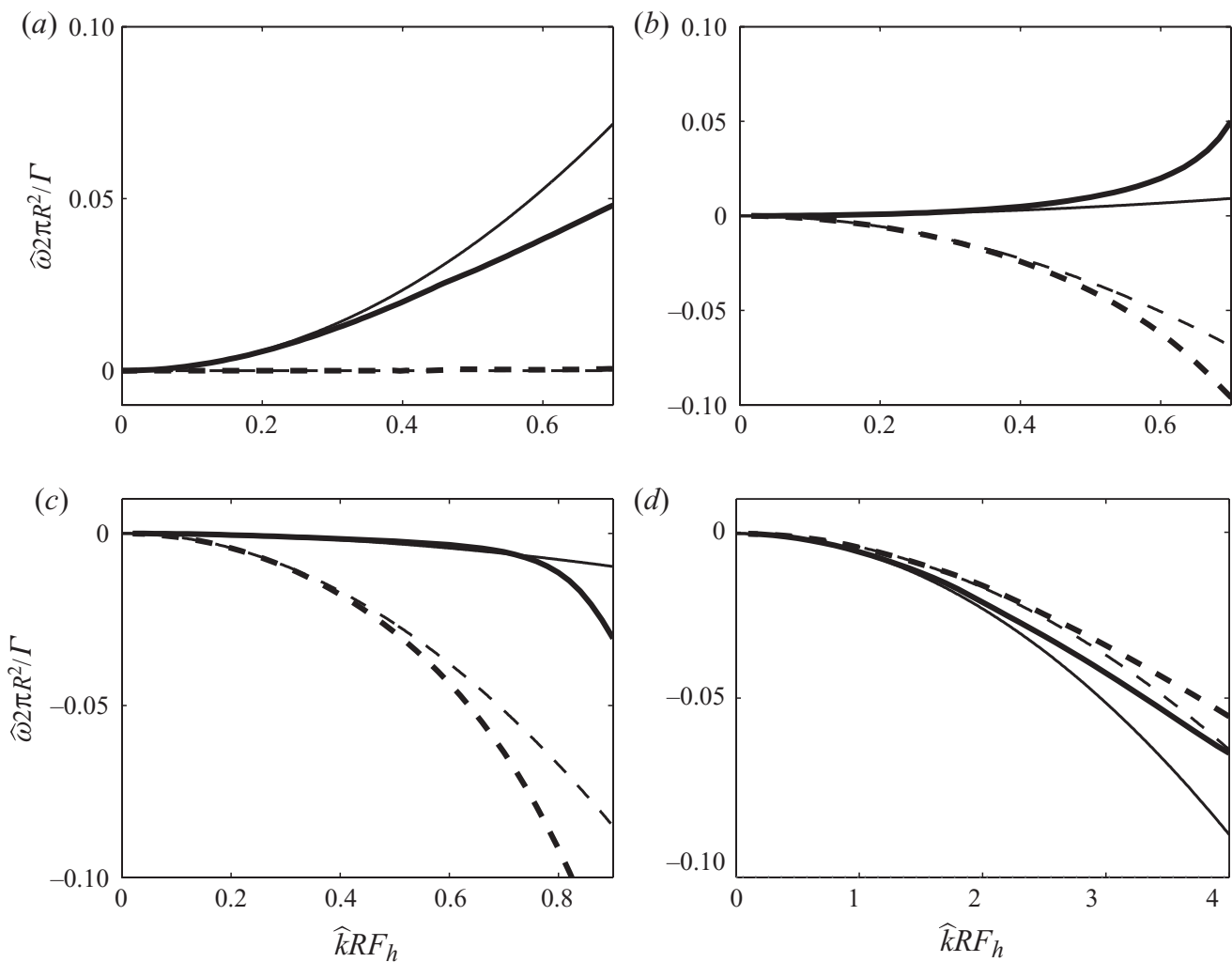

FIGURE 5. Comparison between the self-induction (real part: thin solid line; imaginary part: thin dashed line) for a Lamb-Oseen vortex in a stratified non-rotating fluid and the exact frequency $\omega_{e}$ of the slow bending mode $m=+1$ obtained numerically by a shooting method (real part: thick solid line; imaginary part: thick dashed line) for various Froude numbers: $(a)$ $F_{h}=0.9,(b) F_{h}=1.67,(c) F_{h}=2$ and $(d) F_{h}=10$.

Sipp \& Jacquin (2003). The boundary condition at $r \rightarrow \infty$ is $\hat{u}_{r} \rightarrow 0$ or waves radiate outwards. At $r=0$, the boundary condition is $\partial \hat{u}_{r} / \partial r=0$ since $m=1$. Singular points are bypassed in the complex plane with the rule (3.38).

The results are shown in figure 5 by bold lines. As expected, we see that the agreement with the self-induction function $\omega$ is excellent for small wavenumber $k$. There is a departure as $k$ increases since $\omega$ is only an accurate approximation of $\omega_{e}$ at order $O\left(k^{2}\right)$.

When the Froude number becomes large and $R o=\infty$, one could expect that the self-induction function (4.1) tends to that for homogeneous fluids (cf. (4.2)). However, using (4.9), we see that for large Froude number $F_{h}$ and for $R o=\infty$, (4.1) tends to

$$
\omega=\frac{k^{2} \mathscr{A}}{2} \approx-\frac{\hat{k}^{2} R^{2}}{4}\left(\ln F_{h}+\gamma_{e}-\ln 2+\mathrm{i} \frac{\pi}{2}\right) .
$$

This shows that the real part of the frequency becomes more and more negative as $F_{h}$ increases while the imaginary part is independent of $F_{h}$ when represented as a function of $\hat{k} R$. Therefore, the self-induction (4.1) is always different from (4.2) even when $F_{h} \rightarrow \infty$. In order to resolve this paradox, one has to remember that (4.1) is valid only for small wavenumbers satisfying the condition $(3.71 b)$, i.e. $\hat{k} R F_{h} \ll \max \left(1, \sqrt{F_{h}}\right)$ for $R o=\infty$. Thus, the domain of validity of (4.1) for $F_{h}>1$, 


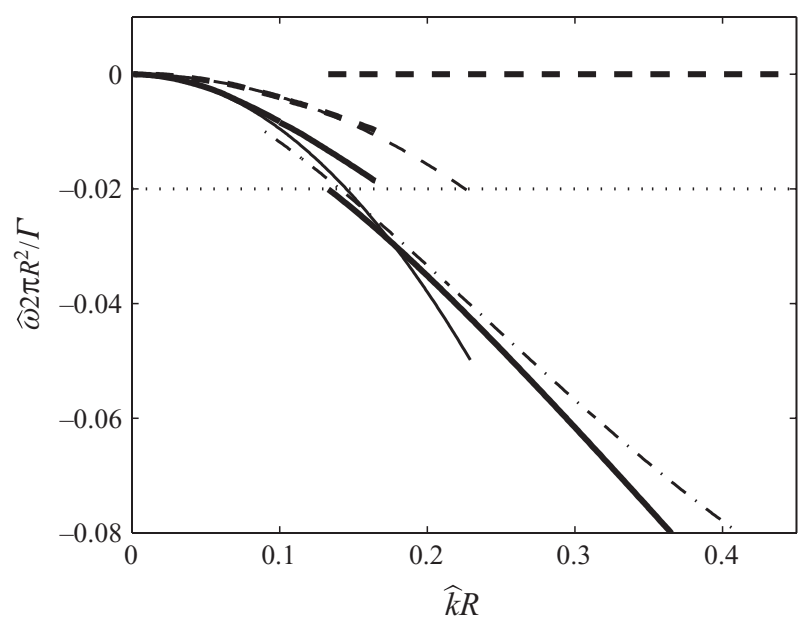

FiguRE 6. Comparison between the self-induction (real part: thin solid line; imaginary part: thin dashed line) for a Lamb-Oseen vortex in a stratified non-rotating fluid for $F_{h}=50$ and the frequency $\omega_{e}$ of the slow bending mode $m=+1$ obtained numerically by a shooting method (real part: thick solid line; imaginary part: thick dashed line). The self-induction in homogeneous fluids $\left(F_{h}=\infty\right)$ is also shown by a dashed-dotted line. The dotted line shows the critical frequency $\omega=-1 / F_{h}$.

i.e. $\hat{k} R \ll 1 / \sqrt{F_{h}}$, shrinks to zero when $F_{h} \rightarrow \infty$. This is equivalent to $|\omega| \ll 1 / F_{h}$. Figure 6 shows this situation for $F_{h}=50$. (Note that $\omega$ and $\omega_{e}$ are plotted as a function of $\hat{k} R$ instead of $\hat{k} F_{h} R$ as before.) We see that the frequency $\omega_{e}$ obtained numerically (bold lines) is discontinuous and exhibits two separate branches, the first for $-1 / F_{h}=-0.02<\omega_{e}<0$, which has a negative imaginary part, and the second for $\omega_{e}<-0.02$, which presents a purely real frequency. The self-induction function (4.1) gives a good approximation of the first branch whereas (4.2) asymptotes the second branch. There is therefore no contradiction between (4.1) and (4.2); they simply apply to two distinct ranges of frequencies: $|\omega| \ll 1 / F_{h}$ and $1 / F_{h} \ll|\omega| \ll 1$, respectively.

Figure 7 shows some further comparisons for a fixed Froude number, $F_{h}=0.5$, but for various Rossby numbers. The agreement between the self-induction $\omega$ and the exact frequency $\omega_{e}$ is always good for low wavenumbers. The upper limit of this range depends on the Rossby number. For larger wavenumbers, the self-induction function (4.1) tends to generally underestimate the exact frequency of the bending wave for negative $R o$ and overestimate it for positive $R o$. We can also clearly see that the self-induction function for $|R o|=O(1)$ is higher for cyclonic vortices $(R o>0)$ than for anticyclonic vortices $(R o<0)$.

\section{Generalization to a basic state with an arbitrary number of vortices}

The generalization of the asymptotic stability analysis of $\S 3$ to a basic flow made of an arbitrary number $\mathscr{N}$ of well-separated vortices presents no particular difficulty and is straightforward from the analysis of two vortices. We assume here the existence of a two-dimensional vortex configuration which is steady in a given reference frame. Our purpose is only to derive the general form of the stability equations for such basic flow. 

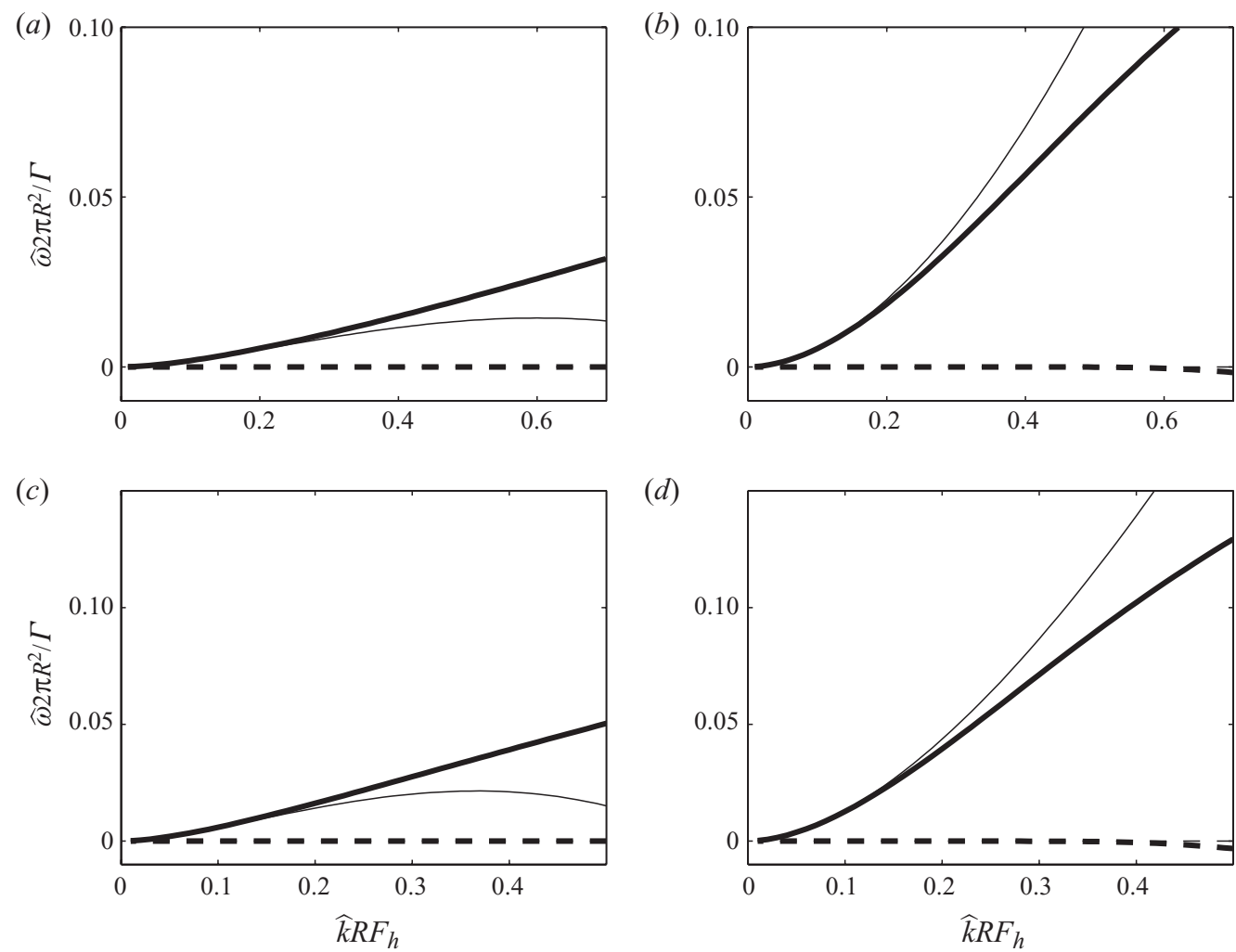

FIGURE 7. Comparison between the self-induction (thin solid line) for a Lamb-Oseen vortex in a strongly stratified $\left(F_{h}=0.5\right)$ and rotating fluid and the exact frequency $\omega_{e}$ of the slow bending mode $m=+1$ obtained numerically by a shooting method (real part: solid thick line; imaginary part: thick dashed line) for various Rossby numbers: (a) $R o=-2.5,(b) R o=2.5$, (c) $R o=-1.25$ and $(d) R o=1.25$.

The approach is the same as carried out in $\S 3$ : we consider the vicinity of a given vortex (labelled $q$ ) and perform an asymptotic expansion with the small strains and small vertical wavenumber. The basic flow near the vortex $q$ (with length nondimensionalized by $R^{(q)}$ and time by $\left.2 \pi R^{(q)^{2}} / \Gamma^{(q)}\right)$ has the same form as (2.12) but with the strain due to each companion vortex $p=\{1,2, \ldots, \mathscr{N}\}(p \neq q)$ :

$$
\psi_{s}=-\frac{1}{\hat{\epsilon}} \sum_{p \neq q} \frac{\Gamma^{(p)}}{4 \pi b^{(q p)^{2}}}\left(r^{2}-h(r)\right) \cos \left(2 \theta-2 \alpha^{(q p)}\right)-\frac{f}{2 \hat{\epsilon}} r^{2},
$$

where $b^{(q p)}$ is the dimensional norm and $\alpha^{(q p)}$ the angle of the vector joining the centre of the vortex $q$ to the centre of vortex $p$ and $f$ is the dimensional angular velocity of the reference frame in which the vortex array is steady. The parameter $\hat{\epsilon}$ is now the typical order of magnitude of the dimensional strains $\Gamma^{(p)} /\left(2 \pi b^{(q p)^{2}}\right)$ exerted by each vortex $p$. Accordingly, the small non-dimensional strain parameter is $\epsilon=\hat{\epsilon} 2 \pi R^{(q)^{2}} / \Gamma^{(q)}$.

The solution of the zeroth-order inner problem of the stability analysis is identical to (3.19). The solution of the first-order inner problem also has the same form as 
(3.44) with $\tilde{\psi}_{1 s}$ still given by (3.42), i.e.

$$
\begin{aligned}
\tilde{\psi}_{1 s}= & \frac{f r}{2 \hat{\epsilon}}\left(\left(\Delta x^{(q)}-\mathrm{i} \Delta y^{(q)}\right) \mathrm{e}^{\mathrm{i} \theta}+\left(\Delta x^{(q)}+\mathrm{i} \Delta y^{(q)}\right) \mathrm{e}^{-\mathrm{i} \theta}\right)-\sum_{p \neq q} \frac{\Gamma^{(p)}}{4 \pi b^{(q p)^{2}} \hat{\epsilon}}\left[\left(\frac{h^{\prime}}{4}+\frac{h}{2 r}-r\right)\right. \\
& \times\left(\left(\Delta x^{(q)}+\mathrm{i} \Delta y^{(q)}\right) \mathrm{e}^{\mathrm{i} \theta-2 \mathrm{i} \alpha^{(q p)}}+\left(\Delta x^{(q)}-\mathrm{i} \Delta y^{(q)}\right) \mathrm{e}^{-\mathrm{i} \theta+2 \mathrm{i} \alpha^{(q p)}}\right) \\
& \left.+\left(\frac{h^{\prime}}{4}-\frac{h}{2 r}\right)\left(\left(\Delta x^{(q)}-\mathrm{i} \Delta y^{(q)}\right) \mathrm{e}^{3 \mathrm{i} \theta-2 \mathrm{i} \alpha^{(q p)}}+\left(\Delta x^{(q)}+\mathrm{i} \Delta y^{(q)}\right) \mathrm{e}^{-3 \mathrm{i} \theta+2 \mathrm{i} \alpha^{(q p)}}\right)\right], \quad(5.2)
\end{aligned}
$$

where $\left(\Delta x^{(q)}, \Delta y^{(q)}\right)$ are the displacements of the vortex $q$. The outer streamfunction (3.67) becomes

$$
\tilde{\psi}_{0}=\beta\left[K_{1}(\beta r)\left(E_{+}^{(q)} \mathrm{e}^{\mathrm{i} \theta}+E_{-}^{(q)} \mathrm{e}^{-\mathrm{i} \theta}\right)+\sum_{p \neq q} \frac{\Gamma^{(p)}}{\Gamma^{(q)}} K_{1}\left(\beta \xi^{(p)}\right)\left(E_{+}^{(p)} \mathrm{e}^{\mathrm{i} \eta^{(p)}}+E_{-}^{(p)} \mathrm{e}^{-\mathrm{i} \eta^{(p)}}\right)\right]
$$

where $\beta=\hat{k} F_{h}^{(q)} R^{(q)} /\left|R o^{(q)}\right|,\left(\xi^{(p)}, \eta^{(p)}\right)$ are the cylindrical coordinates centred on the vortex $p$ and $E_{ \pm}^{(p)}$ are constants.

The matching of the outer and inner streamfunctions then yields the following equations for the displacements of the vortex $q$ :

$$
\begin{aligned}
\frac{\partial}{\partial \hat{t}}\left(\begin{array}{c}
\Delta x^{(q)} \\
\Delta y^{(q)}
\end{array}\right)= & -\sum_{p \neq q} \frac{\Gamma^{(p)}}{2 \pi b^{(q p)^{2}}} \boldsymbol{R}^{(q p)}\left(\begin{array}{cc}
0 & 1 \\
1 & 0
\end{array}\right)\left[\boldsymbol{R}^{(q p)}\right]^{-1}\left(\begin{array}{c}
\Delta x^{(q)} \\
\Delta y^{(q)}
\end{array}\right) \\
& +\sum_{p \neq q} \frac{\Gamma^{(p)}}{2 \pi b^{(q p)^{2}}} \boldsymbol{R}^{(q p)}\left(\begin{array}{cc}
0 & \Psi\left(\hat{\beta} b^{(q p)}\right) \\
\chi\left(\hat{\beta} b^{(q p)}\right) & 0
\end{array}\right)\left[\boldsymbol{R}^{(q p)}\right]^{-1}\left(\begin{array}{c}
\Delta x^{(p)} \\
\Delta y^{(p)}
\end{array}\right) \\
& +\left(f-\frac{\Gamma^{(q)}}{2 \pi R^{(q)^{2}}} \omega_{r}^{(q)}\right)\left(\begin{array}{cc}
0 & 1 \\
-1 & 0
\end{array}\right)\left(\begin{array}{c}
\Delta x^{(q)} \\
\Delta y^{(q)}
\end{array}\right)+\frac{\left|\Gamma^{(q)}\right|}{2 \pi R^{(q)^{2}}} \omega_{i}^{(q)}\left(\begin{array}{c}
\Delta x^{(q)} \\
\Delta y^{(q)}
\end{array}\right),
\end{aligned}
$$

where $\omega^{(q)}=\omega\left(\hat{\beta} R^{(q)}, F_{h}^{(q)}, R o^{(q)}\right)$ and

$$
\boldsymbol{R}^{(q p)}=\left(\begin{array}{cc}
\cos \alpha^{(q p)} & -\sin \alpha^{(q p)} \\
\sin \alpha^{(q p)} & \cos \alpha^{(q p)}
\end{array}\right)
$$

is the rotation matrix. Even if it takes into account the effects of several vortices, (5.4) is similar to (3.84)-(3.85) for the case of two vortices. The only difference is the presence of the rotation matrix $\boldsymbol{R}^{(q p)}$ in (5.4) due to the fact that the Cartesian reference frame is no longer such that the $x$-axis is aligned along the line joining the vortices $q$ and $p$ as in (3.84)-(3.85). However, it can be checked that (5.4) for $\alpha^{(q p)}=0$ and $\mathscr{N}=2$ reduces to (3.84)-(3.85). As before, these equations have the same form as those that would be obtained using vortex filaments with the Biot-Savart law and the cutoff approximation in homogeneous fluids.

\section{Conclusion}

In this paper, we have derived a general theoretical approach to investigate the three-dimensional stability of well-separated vertical columnar vortices with 
respect to long-wavelength bending disturbances in stratified and rotating fluids. Such approach can be seen as the equivalent of the stability analyses based on vortex filaments in homogeneous fluids (Crow 1970; Jimenez 1975; Bristol et al. 2004). While the use of vortex filaments is legitimate in homogeneous fluids due to the Helmholtz and Kelvin theorems, such a concept is not valid in stratified and rotating fluids because the circulation of an individual vortex is not conserved. We have therefore derived the stability equations directly from the Euler equations under the Boussinesq approximation by resorting to a matched asymptotic expansion for well-separated vortices $(R / b \ll 1)$ and small rescaled vertical wavenumber $k R F_{h} \ll \max \left(1, \sqrt{F_{h}}\right) \min (1,|R o|)$. Technically speaking, such asymptotic approach is as accurate as the vortex filament methods in homogeneous fluids: the stability equations are valid up to orders $O\left(R^{2} / b^{2}\right)$ and $O\left(\hat{k}^{2} R^{2}\right)$ in both cases.

The stability equations have been derived in detail in the case of two interacting vortices and generalized to the case of several vortices. The equations are formally identical to those in homogeneous fluids. They express the fact that the bending deformation of a vortex column generally evolves because of three effects: its own self-induced motion as if the other vortices were absent, the strain field generated by the companion vortices and the remote effect of their bending disturbances (mutual induction).

The nature of the fluid, stratified-rotating or homogeneous, appears only in the mutual-induction and self-induction functions. We have derived their expressions for any Rossby number $R o$ and for a wide range of Froude number such that $F_{h} \ll b^{2} / R^{2}$, i.e. when the strain is much smaller than the Brunt-Väisälä frequency. Compared to homogeneous fluids, the most crucial difference is the sign of the selfinduction function: it is positive in stratified and rotating fluids when $F_{h}<1 / \Omega_{\max }$ while it is negative in homogeneous fluids. Furthermore, the self-induction function becomes complex when $F_{h}>1 / \Omega_{\max }$ because the bending disturbances are damped by a viscous critical layer at the radius where the angular velocity of each vortex is equal to the Brunt-Väisälä frequency. Dissipative effects can also be taken into account when there is no critical layer.

The present theory extends in many directions the previous theoretical analyses of the zigzag instability that have been performed only in strongly stratified non-rotating inviscid fluids and for specific basic flows (Billant \& Chomaz 2000b; Otheguy et al. 2007). The study of the stability of vortex pairs in stratified and rotating fluids using the final equations (3.84)-(3.87) is therefore of high interest and should provide a comprehensive understanding of the zigzag instability. Such stability analysis will be carried out in Part 2. The theoretical predictions will be validated against the results of direct numerical stability analyses of co- and counter-rotating vortex pairs. A very good agreement will be found except for co-rotating vortex pairs when the Rossby number has $O(1)$ negative values. The discrepancy comes from the fact that the self-induction is very low for these Rossby number values (see figure 7) so that the balance between strain and self-induction effects is actually achieved only when the vertical wavenumber is no longer small. An improved theory taking into account higher-order three-dimensional effects is developed in Appendix E for this particular range of the Rossby number.

The present theory will also provide a general framework to understand the physical mechanism of the zigzag instability. In particular, the sign reversal of the self-induction function will be shown to explain why vortex pairs are subjected to the zigzag instability in stratified and rotating fluids. 
I would like to thank J. M. Chomaz, A. Deloncle, F. Gallaire, L. Hua, S. Leblanc, S. Le Dizès, P. Otheguy and the referees for their helpful comments and suggestions. I'm very grateful to $\mathrm{P}$. Brancher and A. Antkowiak for providing their viscous Chebyshev spectral stability code.

\section{Appendix A. Critical layer}

In this appendix, we derive the vertical velocity of the zeroth-order problem (\$3.1.1) near the singular radius $r_{c}$ where $\Omega\left(r_{c}\right)=1 / F_{h}^{(l)}$. Near $r_{c}$, the neglected terms, namely the advection by the straining flow, the slow evolution and diffusion of the perturbation are no longer small compared to the leading-order terms. In order to determine the structure of the critical layer, these higher-order effects therefore need to be re-inserted in (3.14) and (3.16) for the vertical velocity $\tilde{u}_{z 0}$ and density $\tilde{\rho}_{0}$ :

$$
\begin{aligned}
F_{h}^{(l)^{2}} \mathscr{P}\left(\tilde{u}_{z 0}\right) & =-\mathrm{i} \tilde{p}_{0}-\tilde{\rho}_{0}+\frac{\delta_{\Gamma}}{R e^{(l)}}\left(F_{h}^{(l)^{2}} \Delta_{h} \tilde{u}_{z 0}-k^{2} \tilde{u}_{z 0}\right), \\
\mathscr{P}\left(\tilde{\rho}_{0}\right) & =\tilde{u}_{z 0}+\frac{\delta_{\Gamma}}{R e^{(l)} S c}\left(\Delta_{h} \tilde{\rho}_{0}-\frac{k^{2}}{F_{h}^{(l)}} \tilde{\rho}_{0}\right),
\end{aligned}
$$

where $\tilde{p}_{0}$ is given by $(3.25)$ and the operator $\mathscr{P}$ is

$$
\mathscr{P}=\Omega \frac{\partial}{\partial \theta}+\epsilon \frac{\partial}{\partial T}+\epsilon \boldsymbol{U}_{s} \cdot \nabla .
$$

For large Reynolds numbers as considered herein, the terms of order $\epsilon$ are generally much larger than diffusive effects. Therefore, at first sight, one could think that only the $O(\epsilon)$ terms would need to be considered near the singularity. However, these terms cannot generally prevent the existence of the singularity. Therefore, both $O(\epsilon)$ terms and viscous terms need to be taken into account. To this end, the operator $\mathscr{P}$ can be first simplified near the critical radius $r_{c}$ by using new coordinates:

$$
\begin{aligned}
& s=r-\frac{\epsilon}{2 \Omega_{c}}\left(r_{c}-\frac{h_{c}}{r_{c}}\right) \cos 2 \theta-\epsilon r_{c 1}, \\
& \alpha=\theta+\frac{\epsilon}{2 \Omega_{c}}\left[\left(1-\frac{h_{c}^{\prime}}{2 r_{c}}\right)-\frac{\Omega_{c}^{\prime}}{2 \Omega_{c}}\left(r_{c}-\frac{h_{c}}{r_{c}}\right)\right] \sin 2 \theta,
\end{aligned}
$$

where the subscript $c$ indicates the value taken at $r_{c}$. The variable $s$ is constant along the streamline of the basic state whose mean radius is $r_{c}$. When the angular velocity $\Omega$ is constant, these coordinates become equivalent to the elliptico-cylindrical coordinates (Waleffe 1990; Mason \& Kerswell 1999) for small ellipticity. The parameter $r_{c 1}$ has been introduced in (A 4) in order to anticipate for a slight shift of the critical radius. With these coordinates, the operator $\mathscr{P}$ becomes

$$
\mathscr{P}=\left(\Omega_{c}+\Omega_{c}^{\prime}\left(s-r_{c}\right)+\epsilon\left(\Omega_{c}^{\prime} r_{c 1}-\tilde{f}\right)\right) \frac{\partial}{\partial \alpha}+\epsilon \frac{\partial}{\partial T}+O\left(\epsilon\left(s-r_{c}\right),\left(s-r_{c}\right)^{2}\right) .
$$

Note that the present analysis assumes $\Omega_{c}^{\prime} \neq 0$. It is therefore not valid when $r_{c}$ is very close to the vortex axis, i.e. when $F_{h}^{(l)}$ is just slightly above the critical value $1 / \Omega_{\max }$. Following classical analysis of viscous critical layer, the viscous effects are taken into account by introducing the local variable

$$
x=\operatorname{Re}^{(l)^{1 / 3}}\left(s-r_{c}\right)
$$


and expanding the local solution in power of $R e^{(l)^{1 / 3}}$ :

$$
\left(\tilde{u}_{z 0}, \tilde{\rho}_{0}\right)=\operatorname{Re}^{(l)^{1 / 3}}\left(\tilde{u}_{z 00}, \tilde{\rho}_{00}\right)+\left(\tilde{u}_{z 01}, \tilde{\rho}_{01}\right)+\cdots .
$$

At leading order, (A 1)-(A 2) become

$$
\begin{aligned}
F_{h}^{(l)^{2}} \Omega_{c} \frac{\partial \tilde{u}_{z 00}}{\partial \alpha} & =-\tilde{\rho}_{00}, \\
\Omega_{c} \frac{\partial \tilde{\rho}_{00}}{\partial \alpha} & =\tilde{u}_{z 00} .
\end{aligned}
$$

Writing the vertical velocity in the form

$$
\tilde{u}_{z 00}=\tilde{W}_{+} C_{+}^{(l)} \mathrm{e}^{\mathrm{i} \alpha}-\tilde{W}_{-} C_{-}^{(l)} \mathrm{e}^{-\mathrm{i} \alpha}
$$

gives the condition of existence of the critical layer: $F_{h}^{(l)^{2}} \Omega_{c}^{2}=1$. At order $\epsilon \operatorname{Re} e^{(l)}{ }^{1 / 3}$, we have

$$
\begin{aligned}
F_{h}^{(l)^{2}}\left[\left(\Omega_{c}^{\prime} r_{c 1}-\tilde{f}\right) \frac{\partial \tilde{u}_{z 00}}{\partial \alpha}+\frac{\partial \tilde{u}_{z 00}}{\partial T}\right] & =0, \\
\left(\Omega_{c}^{\prime} r_{c 1}-\tilde{f}\right) \frac{\partial \tilde{\rho}_{00}}{\partial \alpha}+\frac{\partial \tilde{\rho}_{00}}{\partial T} & =0 .
\end{aligned}
$$

This yields the shift of the critical radius

$$
r_{c 1 \pm}=\frac{1}{\Omega_{c}^{\prime}}\left(\tilde{f} \pm \mathrm{i} \frac{\partial \ln C_{ \pm}^{(l)}}{\partial T}\right)
$$

for the functions $\tilde{W}_{+}$and $\tilde{W}_{-}$, respectively. It can be noticed that $r_{c 1 \pm}$ is complex when the growth rate of $C_{ \pm}^{(l)}$ has a real part, meaning that the critical radius moves slightly off the real $r$-axis. Ât order $O(1)$, we have

$$
\begin{aligned}
F_{h}^{(l)^{2}}\left(\Omega_{c} \frac{\partial \tilde{u}_{z 01}}{\partial \alpha}+\Omega_{c}^{\prime} x \frac{\partial \tilde{u}_{z 00}}{\partial \alpha}\right) & =-\mathrm{i} \tilde{p}_{0}-\tilde{\rho}_{01}+\delta_{\Gamma} F_{h}^{(l)^{2}} \frac{\partial^{2} \tilde{u}_{z 00}}{\partial x^{2}} \\
\Omega_{c} \frac{\partial \tilde{\rho}_{01}}{\partial \alpha}+\Omega_{c}^{\prime} x \frac{\partial \tilde{\rho}_{00}}{\partial \alpha} & =\tilde{u}_{z 01}+\frac{\delta_{\Gamma}}{S c} \frac{\partial^{2} \tilde{\rho}_{00}}{\partial x^{2}} .
\end{aligned}
$$

The pressure given by (3.25) can be rewritten in terms of $\alpha$ :

$$
\tilde{p}_{0}=r_{c} \Omega_{c}\left(\Omega_{c}+R o^{(l)^{-1}}\right)\left(C_{+}^{(l)} \mathrm{e}^{\mathrm{i} \alpha}+C_{-}^{(l)} \mathrm{e}^{-\mathrm{i} \alpha}\right)+O\left(\epsilon, R e^{(l)-1 / 3}\right) .
$$

Combining (A 15) and (A 16) gives

$$
2 \Omega_{c}^{\prime} \times \tilde{W}_{ \pm} \pm \mathrm{i} \delta_{\Gamma}\left(1+\frac{1}{S c}\right) \frac{\partial^{2} \tilde{W}_{ \pm}}{\partial x^{2}}=-r_{c} \Omega_{c} \frac{\Omega_{c}+R o^{(l)}-1}{F_{h}^{(l)^{2}}} .
$$

As shown by Boulanger et al. (2007), the solution of (A 18) which matches the inviscid solution (3.27) for $x \rightarrow \pm \infty$ is

$$
\tilde{W}_{ \pm}(x)= \pm \mathrm{i} \Omega_{c} r_{c} \pi \Lambda \frac{\Omega_{c}+R o^{(l)}-1}{2 F_{h}^{(l)^{2}} \Omega_{c}^{\prime}} \operatorname{Hi}( \pm \mathrm{i} \Lambda x),
$$


where Hi is the Scorer's function (Abramowitz \& Stegun 1965; Drazin \& Reid 1981; Gil et al. 2002) and

$$
\Lambda=-\operatorname{sgn}\left(\Gamma^{(l)} \Omega_{c}^{\prime}\right)\left(\frac{2\left|\Omega_{c}^{\prime}\right|}{1+1 / S c}\right)^{1 / 3} .
$$

The vertical velocity in the neighbourhood of $r_{c}$ is therefore given at leading order by (3.28) when re-scaled, i.e. $W_{ \pm}=R e^{(l)^{1 / 3}} \tilde{W}_{ \pm}$, and re-written in terms of the original variables $(r, \theta)$.

\section{Appendix B. Behaviour of the functions $\mathscr{F}_{ \pm}(r)$ for large $r$}

In order to perform the matching between the inner and outer solutions, we need to obtain the behaviour for large $r$ of the functions $\mathscr{F}_{+}(r)$ defined in (3.45). To do so, we shall use the inviscid expression (3.27) of $W_{ \pm}$for all $r$ but the contour of integration will be deformed in the complex plane when a singularity exists. Thus, the function $\mathscr{F}_{+}(r)$ can be written as

$$
\mathscr{F}_{+}(r)=r \Omega(r)\left[\int_{0}^{r} \eta \Omega(\eta) \frac{\Omega(\eta)+R o^{-1}}{1-F_{h}^{2} \Omega(\eta)^{2}} \mathrm{~d} \eta+\int_{0}^{r} \frac{\mathrm{d} \eta}{\eta^{3} \Omega^{2}(\eta)}\left(I_{1}(\eta)+2 \frac{I_{2}(\eta)}{R o}+\frac{I_{3}(\eta)}{R o^{2}}\right)\right],
$$

where the superscript $(l)$ of the Froude and Rossby numbers has been dropped for simplicity and where

$$
I_{p}(\eta)=\int_{0}^{\eta} \frac{\xi^{3} \Omega(\xi)^{(5-p)}}{1-F_{h}^{2} \Omega(\xi)^{2}} \mathrm{~d} \xi \quad \text { for } \quad p=\{1,2,3\}
$$

The contour of integration is deformed according to the rule (3.38) when a singularity exists. The function $\mathscr{F}_{-}(r)$ has the same expression as $(\mathrm{B} 1)$ except that the contour of integration is deformed in the opposite side of the complex plane. This implies $\mathscr{F}_{-}(r)=\mathscr{F}_{+}^{*}(r)$.

Because $\Omega \sim 1 / r^{2}$ as $r \rightarrow \infty$, the behaviours of the integrals $I_{p}(\eta)$ for $\eta \gg 1$ are

$$
\begin{aligned}
& I_{1}(\eta) \sim \mathscr{A}\left(F_{h}\right)+\frac{1}{4 F_{h}^{2}} \ln \left(1-\frac{F_{h}^{2}}{\eta^{4}}\right), \\
& I_{2}(\eta) \sim \mathscr{B}\left(F_{h}\right)+\frac{1}{4 F_{h}} \ln \left(\frac{\eta^{2}-F_{h}}{\eta^{2}+F_{h}}\right), \\
& I_{3}(\eta) \sim \mathscr{D}\left(F_{h}\right)+\frac{1}{4} \ln \left(\eta^{4}-F_{h}^{2}\right),
\end{aligned}
$$

where $(\mathscr{A}, \mathscr{B}, \mathscr{D})$ are constants depending on $F_{h}$ given by

$$
\begin{aligned}
& \mathscr{A}\left(F_{h}\right)=\lim _{\eta_{0} \rightarrow \infty} \int_{0}^{\eta_{0}} \frac{\xi^{3} \Omega(\xi)^{4}}{1-F_{h}^{2} \Omega(\xi)^{2}} \mathrm{~d} \xi-\frac{1}{4 F_{h}^{2}} \ln \left(1-\frac{F_{h}^{2}}{\eta_{0}^{4}}\right), \\
& \mathscr{B}\left(F_{h}\right)=\lim _{\eta_{0} \rightarrow \infty} \int_{0}^{\eta_{0}} \frac{\xi^{3} \Omega(\xi)^{3}}{1-F_{h}^{2} \Omega(\xi)^{2}} \mathrm{~d} \xi-\frac{1}{4 F_{h}} \ln \left(\frac{\eta_{0}^{2}-F_{h}}{\eta_{0}^{2}+F_{h}}\right), \\
& \mathscr{D}\left(F_{h}\right)=\lim _{\eta_{0} \rightarrow \infty} \int_{0}^{\eta_{0}} \frac{\xi^{3} \Omega(\xi)^{2}}{1-F_{h}^{2} \Omega(\xi)^{2}} \mathrm{~d} \xi-\frac{1}{4} \ln \left(\eta_{0}^{4}-F_{h}^{2}\right) .
\end{aligned}
$$

In practice, the value of $\eta_{0}$ in the limits does not need to be very large. The limiting values are reached as soon as $\eta_{0}$ lies in the region where the basic vorticity becomes 
negligible: $\zeta\left(\eta_{0}\right) \approx 0$. Note that these formulae can be further simplified if $\eta_{0}$ is chosen such that $\eta_{0} \gg \sqrt{F_{h}}$.

Using these asymptotic behaviours, we see that $\mathscr{F}_{+}(r)$ behaves asymptotically for large $r$ like

$$
\begin{aligned}
\mathscr{F}_{+}(r) \sim \frac{r}{2 R o^{2}}[\ln r & +\delta\left(F_{h}, R o\right)-\frac{1}{2}+\left(\frac{R o^{2}}{4 F_{h}^{2}}+\frac{1}{4}\right) \ln \left(1-\frac{F_{h}^{2}}{r^{4}}\right) \\
+ & \left.\left(\frac{R o}{2 F_{h}}-\frac{F_{h}}{4 r^{2}}+\frac{R o^{2}}{4 F_{h} r^{2}}\right) \ln \left(\frac{r^{2}-F_{h}}{r^{2}+F_{h}}\right)\right]+O\left(\frac{1}{r}\right),
\end{aligned}
$$

where $\delta\left(F_{h}, R o\right)=\mathscr{D}\left(F_{h}\right)+2 \operatorname{Ro\mathscr {B}}\left(F_{h}\right)+R o^{2} \mathscr{A}\left(F_{h}\right)$. Since the matching requires the behaviour of $\mathscr{F}_{+}(r)$ for $r \gg \sqrt{F_{h}}$, the previous expression can be further simplified as

$$
\mathscr{F}_{+}(r) \sim \frac{r}{2 R o^{2}}\left[\ln r+\delta\left(F_{h}, R o\right)-\frac{1}{2}\right]+O\left(\frac{1}{r}\right) .
$$

Using (B 6)-(B 8), $\delta$ can also be written in a compact form

$$
\delta\left(F_{h}, R o\right)=\lim _{\eta_{0} \rightarrow \infty} \int_{0}^{\eta_{0}} \xi^{3} \Omega(\xi)^{2} \frac{(R o \Omega(\xi)+1)^{2}}{1-F_{h}^{2} \Omega(\xi)^{2}} \mathrm{~d} \xi-\ln \eta_{0} .
$$

This expression shows that $\delta$ will be minimum for negative Rossby number of order unity and positive for large absolute values of the Rossby number independently of the angular velocity profile $\Omega$ in the case of a strongly stratified fluid $F_{h}<1 / \Omega_{\max }$. Note that it is more convenient to use (B 6)-(B 8) than (B 11) because the latter formula needs to be computed for each $R o$.

\section{Appendix C. Rankine vortex}

The parameters $(\mathscr{A}, \mathscr{B}, \mathscr{D})$ for the Rankine vortex, i.e. $\Omega=1$ for $r<1$ and $\Omega=1 / r^{2}$ for $r>1$, can be obtained analytically:

$$
\left.\begin{array}{l}
\mathscr{A}\left(F_{h}\right)=\frac{1}{4\left(1-F_{h}^{2}\right)}-\frac{1}{4 F_{h}^{2}} \ln \left(1-F_{h}^{2}\right), \\
\mathscr{B}\left(F_{h}\right)=\frac{1}{4\left(1-F_{h}^{2}\right)}+\frac{1}{4 F_{h}} \ln \left(\frac{1+F_{h}}{1-F_{h}}\right), \\
\mathscr{D}\left(F_{h}\right)=\frac{1}{4\left(1-F_{h}^{2}\right)}-\frac{1}{4} \ln \left(1-F_{h}^{2}\right) .
\end{array}\right\}
$$

The logarithmic function should be interpreted in the following manner for $F_{h}>1$ : $\ln \left(1-F_{h}\right)=\ln \left|1-F_{h}\right|+\mathrm{i} \pi$. The values for $F_{h}=0$ fully agree with the results obtained by Billant, Dritschel \& Chomaz (2006) for the Moore-Saffman elliptical vortex in the limit of zero ellipticity (which then reduces to the Rankine vortex) in a strongly stratified and rotating fluid.

\section{Appendix D. Viscous effects}

When there is no critical layer, i.e. for $F_{h}^{(l)}<1 / \Omega_{\max }$, the viscous effects can be easily taken into account at leading order by further expanding each term of the expansion (3.8)-(3.11) with the inverse of the Reynolds number, e.g. for the horizontal velocity

$$
\tilde{\boldsymbol{u}}_{h}=\tilde{\boldsymbol{u}}_{h 0}+\frac{\delta_{\Gamma}}{R \boldsymbol{e}^{(l)}} \tilde{\boldsymbol{u}}_{h 0}^{\nu 1}+\cdots+\epsilon\left(\tilde{\boldsymbol{u}}_{h 1}+\frac{\delta_{\Gamma}}{\operatorname{Re}^{(l)}} \tilde{\boldsymbol{u}}_{h 1}^{\nu 1}+\cdots\right)+\cdots,
$$


where the superscript $\nu 1$ denotes the first viscous correction of each order in $\epsilon$. The other perturbation quantities are expanded similarly. An additional viscous slow timescale also needs to be introduced: $T^{v}=\epsilon t \delta_{\Gamma} / \operatorname{Re}^{(l)}$.

Inserting the expansion (D 1) in (3.2)-(3.5) with the viscous and diffusive terms re-introduced gives the following at the first viscous order $O\left(1 / \operatorname{Re}^{(l)}\right)$ :

$$
\begin{aligned}
\Omega \frac{\partial \tilde{u}_{r 0}^{\nu 1}}{\partial \theta}-\left(2 \Omega+\frac{1}{R o^{(l)}}\right) \tilde{u}_{\theta 0}^{\nu 1} & =-\frac{\partial \tilde{p}_{0}^{\nu 1}}{\partial r}+\Delta_{h} \tilde{u}_{r 0}-\frac{\tilde{u}_{r 0}}{r^{2}}-\frac{2}{r^{2}} \frac{\partial \tilde{u}_{\theta 0}}{\partial \theta} \\
\Omega \frac{\partial \tilde{u}_{\theta 0}^{\nu 1}}{\partial \theta}+\left(\zeta+\frac{1}{R o^{(l)}}\right) \tilde{u}_{r 0}^{\nu 1} & =-\frac{1}{r} \frac{\partial \tilde{p}_{0}^{\nu 1}}{\partial \theta}+\Delta_{h} \tilde{u}_{\theta 0}-\frac{\tilde{u}_{\theta 0}}{r^{2}}+\frac{2}{r^{2}} \frac{\partial \tilde{u}_{r 0}}{\partial \theta} \\
F_{h}^{(l)^{2}} \Omega \frac{\partial \tilde{u}_{z 0}^{\nu 1}}{\partial \theta} & =-\mathrm{i} \tilde{p}_{0}^{\nu 1}-\tilde{\rho}_{0}^{\nu 1}+F_{h}^{(l)^{2}} \Delta_{h} \tilde{u}_{z 0} \\
\frac{1}{r} \frac{\partial r \tilde{u}_{r 0}^{\nu 1}}{\partial r}+\frac{1}{r} \frac{\partial \tilde{u}_{\theta 0}^{\nu 1}}{\partial \theta} & =0 \\
\Omega \frac{\partial \tilde{\rho}_{0}^{\nu 1}}{\partial \theta} & =\tilde{u}_{z 0}^{\nu 1}+\frac{1}{S c} \Delta_{h} \tilde{\rho}_{0} .
\end{aligned}
$$

By writing the horizontal-velocity perturbation in the form $\tilde{\boldsymbol{u}}_{h 0}^{\nu 1}=-\nabla \times\left(\tilde{\psi}_{0}^{\nu 1} \boldsymbol{e}_{z}\right)$, the streamfunction and pressure can be found as

$$
\left[\begin{array}{c}
\tilde{\psi}_{0}^{\nu 1} \\
\tilde{p}_{0}^{\nu 1}
\end{array}\right]=\mathrm{i}\left[\begin{array}{c}
\varphi_{\nu} \\
P_{v}
\end{array}\right]\left(C_{+}^{(l)} \mathrm{e}^{\mathrm{i} \theta}-C_{-}^{(l)} \mathrm{e}^{-\mathrm{i} \theta}\right)
$$

with

$$
\varphi_{\nu}=-r \Omega \int_{\infty}^{r} \frac{1}{\eta \Omega^{2}}\left(\zeta^{\prime \prime}-\frac{\zeta^{\prime}}{\eta}\right) \mathrm{d} \eta, \quad P_{v}=\left(\Omega+R o^{(l)-1}\right) \varphi_{v}-\zeta^{\prime} . \quad(\mathrm{D} 8 a, b)
$$

Note that the integration constants in (D $8 a$ ) have been chosen so that the solution is non-singular at $r=0$ and decays faster than $1 / r$ for large radius, i.e. faster than the leading-order solution (3.19). The vertical velocity is then obtained in the form

$$
\tilde{u}_{z 0}^{v 1}=\mathrm{i} W_{v}\left(C_{+}^{(l)} \mathrm{e}^{\mathrm{i} \theta}+C_{-}^{(l)} \mathrm{e}^{-\mathrm{i} \theta}\right),
$$

with

$$
W_{\nu}=\frac{\Omega\left(F_{h}^{(l)^{2}} \Delta_{h 1} W^{i}+P_{\nu}\right)+S c^{-1} \Delta_{h 1}\left(W^{i} / \Omega\right)}{1-F_{h}^{(l)^{2}} \Omega^{2}},
$$

where $\Delta_{h 1} \equiv \mathrm{d}^{2} / \mathrm{d} r^{2}+(1 / r) \mathrm{d} / \mathrm{d} r-1 / r^{2}$ and $W^{i}$ is given by (3.27). The vertical-velocity amplitude $W_{v}$ decays like $1 / r^{5}$ for large radius and like $r$ for small radius.

At order $O\left(\epsilon / R e^{(l)}\right)$, the equations for the vertical vorticity and the divergence equation are

$$
\begin{gathered}
\Omega \frac{\partial \tilde{\zeta}_{1}^{\nu 1}}{\partial \theta}+\tilde{u}_{r 1}^{\nu 1} \zeta^{\prime}=-\frac{\partial \tilde{\zeta}_{0}}{\partial T^{\nu}}-\boldsymbol{U}_{s} \cdot \nabla \tilde{\zeta}_{0}^{\nu 1}-\tilde{\boldsymbol{u}}_{h 0}^{\nu 1} \cdot \nabla \Delta \psi_{s}+\mathrm{i} \frac{k^{2}}{\epsilon}\left(\frac{1}{R o^{(l)}}+\zeta\right) \tilde{u}_{z 0}^{\nu 1}-\frac{k^{2}}{\epsilon F_{h}^{(l)}{ }^{2}} \tilde{\zeta}_{0}+\Delta_{h} \tilde{\zeta}_{1} \\
\frac{1}{r} \frac{\partial r \tilde{u}_{r 1}^{\nu 1}}{\partial r}+\frac{1}{r} \frac{\partial \tilde{u}_{\theta 1}^{\nu 1}}{\partial \theta}+\mathrm{i} \frac{k^{2}}{\epsilon} \tilde{u}_{z 0}^{\nu 1}=0 .
\end{gathered}
$$


Following the same method as for the first-order inviscid problem, the horizontal velocity is sought in the form

$$
\tilde{\boldsymbol{u}}_{h 1}^{\nu 1}=-\nabla \times\left(\tilde{\psi}_{1}^{\nu 1} \boldsymbol{e}_{z}\right)+\nabla_{h} \tilde{\Phi}_{1}^{\nu 1} .
$$

The divergence equation gives the potential

$$
\tilde{\Phi}_{1}^{\nu 1}=\frac{k^{2}}{\epsilon} \mathscr{H}_{\nu}(r)\left(C_{+}^{(l)} \mathrm{e}^{\mathrm{i} \theta}+C_{-}^{(l)} \mathrm{e}^{-\mathrm{i} \theta}\right),
$$

with

$$
\mathscr{H}_{\nu}(r)=\frac{r}{2} \int_{\infty}^{r} W_{\nu}(\eta) \mathrm{d} \eta-\frac{1}{2 r} \int_{0}^{r} \eta^{2} W_{\nu}(\eta) \mathrm{d} \eta,
$$

where the limits of integration have been chosen so that $\tilde{\Phi}_{1}^{v 1}$ is not singular at $r=0$ and vanishes as $r \rightarrow \infty$ for finite Froude number $F_{h}$. The solution of (D 11) can then be found in the form

$$
\begin{aligned}
\tilde{\psi}_{1}^{\nu 1}= & \mathscr{S}_{\nu}(r)\left(C_{-}^{(l)} \mathrm{e}^{\mathrm{i} \theta}-C_{+}^{(l)} \mathrm{e}^{-\mathrm{i} \theta}\right)-\mathrm{i} r \Omega \int_{0}^{r} \frac{1}{\Omega^{2}}\left(\frac{\partial \tilde{\zeta}_{1+} / \eta}{\partial \eta} \mathrm{e}^{\mathrm{i} \theta}-\frac{\partial \tilde{\zeta}_{1-} / \eta}{\partial \eta} \mathrm{e}^{-\mathrm{i} \theta}\right) \mathrm{d} \eta \\
& +\mathrm{i}\left[\tilde{f} r \Omega \int_{\infty}^{r} \frac{1}{\Omega^{2}} \frac{\partial \phi_{\nu} / \eta}{\partial \eta} \mathrm{d} \eta-r \frac{k^{2}}{\epsilon F_{h}^{(l)^{2}}}+\frac{k^{2}}{\epsilon}\left(\mathscr{F}_{\nu}-r \mathscr{H}_{\nu}^{\prime}\right)\right]\left(C_{+}^{(l)} \mathrm{e}^{\mathrm{i} \theta}-C_{-}^{(l)} \mathrm{e}^{-\mathrm{i} \theta}\right) \\
& -\mathrm{i} r\left(\frac{\partial C_{+}^{(l)}}{\partial T^{\nu}} \mathrm{e}^{\mathrm{i} \theta}-\frac{\partial C_{-}^{(l)}}{\partial T^{\nu}} \mathrm{e}^{-\mathrm{i} \theta}\right)+(\cdots) \mathrm{e}^{ \pm 3 i \theta}
\end{aligned}
$$

where $\tilde{\zeta}_{1 \pm}$ represent the azimuthal components $\exp ( \pm \mathrm{i} \theta)$ of $\tilde{\zeta}_{1}$, and

$$
\begin{aligned}
& \mathscr{S}_{\nu}(r)=-\frac{r \Omega}{4} \int_{\infty}^{r} \frac{\mathrm{d} \eta}{\eta^{3} \Omega^{2}}\left[\phi_{\nu}\left(\eta h^{\prime \prime}-4 \frac{h}{\eta}+2 \eta\right)-\eta \phi_{v}^{\prime}\left(2 \eta-h^{\prime}\right)+2 \eta\left(\eta^{2}-h\right) \phi_{\nu}^{\prime \prime}\right], \\
& \mathscr{F}_{\nu}(r)=r \Omega\left[\int_{0}^{r} \frac{W_{v}}{\Omega} \mathrm{d} \eta+\int_{0}^{r} \frac{\mathrm{d} \eta}{\eta^{3} \Omega^{2}(\eta)} \int_{0}^{\eta}\left(\Omega(\xi)+\frac{1}{R o^{(l)}}\right) \xi^{2} W_{\nu}(\xi) \mathrm{d} \xi\right] .
\end{aligned}
$$

The function $\mathscr{S}_{v}$ goes to zero for $r \rightarrow \infty$ while the behaviour of $\mathscr{F}_{v}$ for large $r$ is $\mathscr{F}_{v} \sim \mathscr{E} r / 2$, where $\mathscr{E}$ is given by

$$
\mathscr{E}\left(F_{h}^{(l)}, R o^{(l)}, S c\right)=\int_{0}^{\infty}\left(\Omega(\xi)+\frac{1}{R o^{(l)}}\right) \xi^{2} W_{v}(\xi) \mathrm{d} \xi .
$$

This parameter can be further decomposed in the convenient form

$$
\mathscr{E}\left(F_{h}, R o, S c\right)=\mathscr{E}_{1}\left(F_{h}\right)+\frac{\mathscr{E}_{2}\left(F_{h}\right)}{R o}+\frac{\mathscr{E}_{3}\left(F_{h}\right)}{R o^{2}}+\frac{1}{S c}\left[\mathscr{E}_{4}\left(F_{h}\right)+\frac{\mathscr{E}_{5}\left(F_{h}\right)}{R o}+\frac{\mathscr{E}_{6}\left(F_{h}\right)}{R o^{2}}\right] .
$$

Each parameter $\mathscr{E}_{i}$ is shown in figure $8(a)$ as a function of the Froude number for the case of the Lamb-Oseen profile (4.7). The parameters $\mathscr{E}_{i}$ are almost independent of the Froude number up to $F_{h} \approx 0.8$ and then vary abruptly around $F_{h} \approx 0.9$. From (3.44) and (3.77), the behaviour of $\tilde{\zeta}_{1 \pm}$ for large $r$ is $\tilde{\zeta}_{1 \pm} \sim C_{ \pm}^{(l)} \beta^{2} /(\epsilon r)$. Therefore, the behaviour of (D 16) for large $r$ is

$$
\tilde{\psi}_{1}^{\nu 1} \sim-\mathrm{i} r\left(\frac{\partial C_{+}^{(l)}}{\partial T^{\nu}} \mathrm{e}^{\mathrm{i} \theta}-\frac{\partial C_{-}^{(l)}}{\partial T^{\nu}} \mathrm{e}^{-\mathrm{i} \theta}\right)-\mathrm{i} r \frac{k^{2} \mathscr{V}}{\epsilon F_{h}^{(l)^{2}}}\left(C_{+}^{(l)} \mathrm{e}^{\mathrm{i} \theta}-C_{-}^{(l)} \mathrm{e}^{-\mathrm{i} \theta}\right)+(\cdots) \mathrm{e}^{ \pm 3 i \theta},
$$



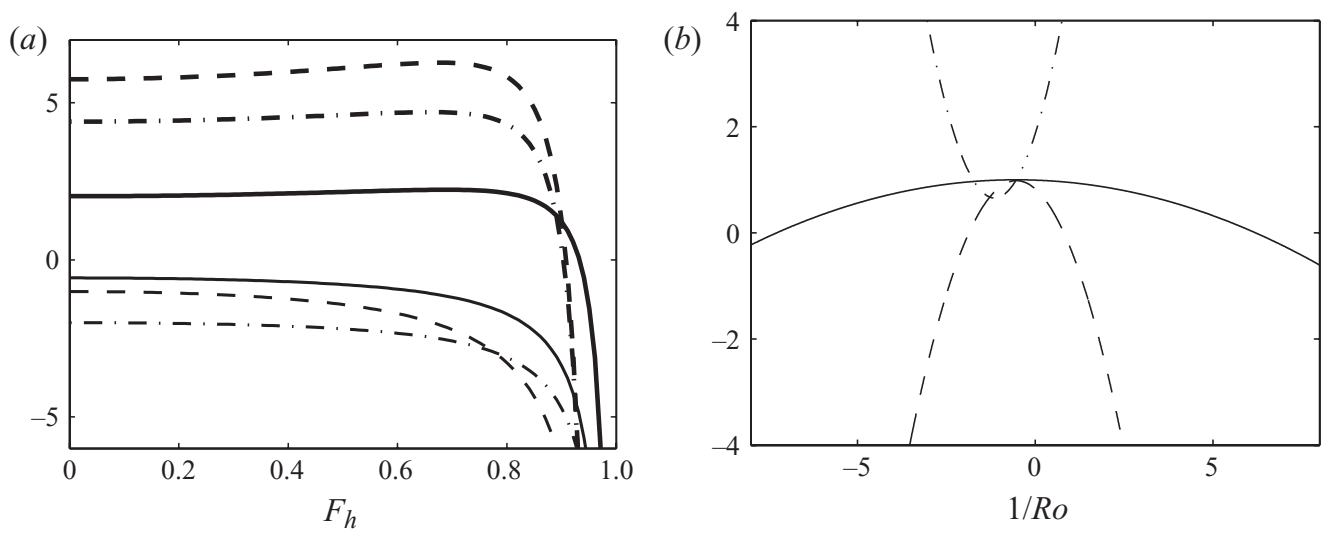

Figure 8. (a) Parameters $\mathscr{E}_{i}$ as a function of the Froude number $F_{h}$ for the Lamb-Oseen vortex (4.7). $\mathscr{E}_{1}(-), \mathscr{E}_{2}(---)$ and $\mathscr{E}_{3}(-\cdot-)$ are shown by bold lines and $\mathscr{E}_{4}(-)$, $\mathscr{E}_{5}$ $(---)$ and $\mathscr{E}_{6}(-\cdot-)$ by thin lines. $(b)$ Coefficient $\mathscr{V}$ describing viscous and diffusive effects as a function of $1 / R o$ for $S c=1$ and $F_{h}=0.1(-), F_{h}=0.5(---)$ and $F_{h}=0.9\left(-^{\cdot-}\right)$.

where

$$
\mathscr{V}=1-\frac{F_{h}^{(l)^{2}}}{R o^{(l)^{2}}}-F_{h}^{(l)^{2}} \frac{\mathscr{E}}{2}
$$

It is straightforward to see that the outer solution at order $O\left(\epsilon / R e^{(l)}\right)$ does not contain any term growing linearly with $r$. Therefore, the matching of (D21) with the outer solution imposes

$$
\frac{\partial C_{ \pm}^{(l)}}{\partial T^{\nu}}=-\frac{k^{2} \mathscr{V}}{\epsilon F_{h}^{(l)}} C_{ \pm}^{(l)} .
$$

When this slow viscous evolution is rescaled and added to the inviscid evolution equations (3.80)-(3.81), the self-induction function is modified according to (3.88). The first term of the coefficient $\mathscr{V}$ defined in (D22) corresponds to the vertical viscous dissipation of the leading order displacement perturbation (3.19). This term is always stabilizing and becomes dominant when $F_{h} \rightarrow 0$ for finite Rossby number. The second term is always destabilizing and comes from the horizontal viscous dissipation of the perturbation at order $\epsilon$. Finally, the third term, which depends on $\mathscr{E}$, describes the horizontal diffusion of the leading-order vertical velocity (3.26) and the associated density field $\tilde{\rho}_{0}$. This term also contains the leading three-dimensional effect of the leading-order horizontal viscous flow (D 8), which turns out to be destablizing. Thus, a striking feature is that the coefficient $\mathscr{V}$ can be negative, i.e. viscous effects can be destablizing depending on the Froude, Rossby and Schmidt numbers. As shown in figure $8(b)$, this occurs typically for $|R o| \lesssim F_{h}$ when $F_{h} \lesssim 0.8$. Finally, it is worth noting that the straining flow plays no role at this order. The formula (D23) has been successfully checked for various cases against numerical results obtained for a single Lamb-Oseen vortex by means of the viscous Chebyshev spectral stability code written by Antkowiak \& Brancher (2007) and adapted to stratified and rotating fluids. 


\section{Appendix E. A combined asymptotical-numerical approach}

In Part 2, it will be found that the results of the asymptotic stability equations (3.84)-(3.87) are in very good agreement with those of direct numerical stability analyses except for equal-strength co-rotating vortex pairs when the Rossby number is around $R o \approx-2$. In this particular range of the Rossby number, the self-induction function remains very small even for $k=O(1)$ (see figures $7 a$ and $7 c$ ). In other words, the bending waves are rotating very slowly. Thus, strain and self-induction effects have the same order $\omega=O(\epsilon)$ only when $k$ is no longer very small. This situation calls for considering higher-order three-dimensional effects in the matched asymptotic analysis. However, this is quite difficult as the algebra becomes extremely lengthy. It is easier to take into account the higher orders in $k$ by using the exact eigenmode of the bending waves of a single vortex computed numerically. Nevertheless, this requires to compute by an asymptotic method the interaction between these waves and the strain and the mutual-induction effects for finite $k$. We have thus developed a combined asymptotical-numerical approach for this specific case based on the ad hoc assumptions that $\epsilon \ll 1$ and that the frequency of the bending waves is of the same order as the strain, $\omega_{e}=O(\epsilon)$, while the vertical wavenumber is finite, $k=O(1)$.

The perturbations are thus expanded with $\epsilon$ only:

$$
[\tilde{\boldsymbol{u}}, \tilde{\rho}]=\left[\tilde{\boldsymbol{u}}_{0}, \tilde{\rho}_{0}\right]+\epsilon\left[\tilde{\boldsymbol{u}}_{1}, \tilde{\rho}_{1}\right]+\cdots
$$

Note that the vertical velocity and the density of the perturbation are not scaled by $k$ in contrast to (3.10) and (3.11). The problem at leading order near the vortex $(l)$ gives (4.11) for the waves on an axisymmetric vortex. At this stage, the frequency $\omega_{e}^{(l)}$ cannot be neglected since otherwise (4.11) would not have a solution for finite $k$. The solutions are taken as

$$
\left[\tilde{\boldsymbol{u}}_{0}, \tilde{\rho}_{0}\right](r, \theta, t)=\hat{C}_{+}^{(l)}(T)\left[\hat{\boldsymbol{u}}_{0+}, \hat{\rho}_{0+}\right](r) \mathrm{e}^{-\mathrm{i} \omega_{e}^{(l)} t+\mathrm{i} \theta}+\hat{C}_{-}(T)^{(l)}\left[\hat{\boldsymbol{u}}_{0-}, \hat{\rho}_{0-}\right](r) \mathrm{e}^{\mathrm{i} \omega_{e}^{(l) *} t-\mathrm{i} \theta},
$$

where the amplitudes $\hat{C}_{ \pm}^{(l)}$ are assumed to depend on a slow time $T=\epsilon t$. The following relation between the eigenfunctions for $m=1$ and $m=-1$ holds: $\left[\hat{u}_{r 0-}, \hat{u}_{\theta 0-}, \hat{u}_{z 0-}, \hat{\rho}_{0-}\right]=\left[\hat{u}_{r 0+}^{*}, \hat{u}_{\theta 0+}^{*},-\hat{u}_{z 0+}^{*},-\hat{\rho}_{0+}^{*}\right]$.

At first order, the problem is

$$
\begin{aligned}
\frac{\partial \tilde{u}_{r 1}}{\partial t}+\Omega \frac{\partial \tilde{u}_{r 1}}{\partial \theta}-\left(2 \Omega+\frac{1}{R o^{(l)}}\right) \tilde{u}_{\theta 1} & =-\frac{\partial \tilde{p}_{1}}{\partial r}+N^{(1)}+R^{(1)}, \\
\frac{\partial \tilde{u}_{\theta 1}}{\partial t}+\Omega \frac{\partial \tilde{u}_{\theta 1}}{\partial \theta}+\left(\zeta+\frac{1}{R o^{(l)}}\right) \tilde{u}_{r 1} & =-\frac{1}{r} \frac{\partial \tilde{p}_{1}}{\partial \theta}+N^{(2)}+R^{(2)}, \\
F_{h}^{(l)}\left(\frac{\partial \tilde{u}_{z 1}}{\partial t}+\Omega \frac{\partial \tilde{u}_{z 1}}{\partial \theta}\right) & =-\mathrm{i} k \tilde{p}_{1}-\tilde{\rho}_{1}+N^{(3)}+R^{(3)}, \\
\frac{1}{r} \frac{\partial r \tilde{u}_{r 1}}{\partial r}+\frac{1}{r} \frac{\partial \tilde{u}_{\theta 1}}{\partial \theta}+\mathrm{i} k \tilde{u}_{z 1} & =0 \\
\frac{\partial \tilde{\rho}_{1}}{\partial t}+\Omega \frac{\partial \tilde{\rho}_{1}}{\partial \theta} & =\tilde{u}_{z 1}+N^{(4)}+R^{(4)},
\end{aligned}
$$


where the forcing terms $N=\left(N^{(1)}, N^{(2)}, N^{(3)}, N^{(4)}\right)$ correspond to the effect of the straining flow $\boldsymbol{U}_{s}$ and the slow time evolution:

$$
\begin{aligned}
N^{(1)}= & -U_{r s} \frac{\partial \tilde{u}_{r 0}}{\partial r}-\tilde{u}_{r 0} \frac{\partial U_{r s}}{\partial r}-\frac{U_{\theta s}}{r} \frac{\partial \tilde{u}_{r 0}}{\partial \theta}-\frac{\tilde{u}_{\theta 0}}{r} \frac{\partial U_{r s}}{\partial \theta}+2 \frac{U_{\theta s} \tilde{u}_{\theta 0}}{r}+2 \tilde{f} \tilde{u}_{\theta 0}-\frac{\partial \tilde{u}_{r 0}}{\partial T}, \\
N^{(2)}= & -U_{r s} \frac{\partial \tilde{u}_{\theta 0}}{\partial r}-\tilde{u}_{r 0} \frac{\partial U_{\theta s}}{\partial r}-\frac{U_{\theta s}}{r} \frac{\partial \tilde{u}_{\theta 0}}{\partial \theta}-\frac{\tilde{u}_{\theta 0}}{r} \frac{\partial U_{\theta s}}{\partial \theta}-\frac{U_{\theta s} \tilde{u}_{r 0}}{r} \\
& -\frac{U_{r s} \tilde{u}_{\theta 0}}{r}-2 \tilde{f} \tilde{u}_{r 0}-\frac{\partial \tilde{u}_{\theta 0}}{\partial T}, \\
N^{(3)}= & F_{h}^{(l)}{ }^{2}\left(-U_{r s} \frac{\partial \tilde{u}_{z 0}}{\partial r}-\frac{U_{\theta s}}{r} \frac{\partial \tilde{u}_{z 0}}{\partial \theta}-\frac{\partial \tilde{u}_{z 0}}{\partial T}\right), \\
N^{(4)}= & -U_{r s} \frac{\partial \tilde{\rho}_{z 0}}{\partial r}-\frac{U_{\theta s}}{r} \frac{\partial \tilde{\rho}_{z 0}}{\partial \theta}-\frac{\partial \tilde{\rho}_{z 0}}{\partial T},
\end{aligned}
$$

and the terms $\boldsymbol{R}=\left(R^{(1)}, R^{(2)}, R^{(3)}, R^{(4)}\right)$ correspond to mutual-induction effects. These effects will be considered later and, for the moment, we set $\boldsymbol{R}=0$.

Given the forms of the straining flow (2.13) and the leading-order perturbation (E 2), the forcing term $N$ can be decomposed as

$$
\begin{aligned}
\boldsymbol{N}= & \hat{C}_{+}^{(l)} \boldsymbol{S}_{+3} \exp \left(3 \mathrm{i} \theta-\mathrm{i} \omega_{e} t\right)+\hat{C}_{-}^{(l)} \boldsymbol{S}_{-3} \exp \left(-3 \mathrm{i} \theta+\mathrm{i} \omega_{e}^{*} t\right)+\hat{C}_{-}^{(l)} \boldsymbol{S}_{+} \exp \left(\mathrm{i} \theta+\mathrm{i} \omega_{e}^{*} t\right) \\
& +\hat{C}_{+}^{(l)} \boldsymbol{S}_{-} \exp \left(-\mathrm{i} \theta-\mathrm{i} \omega_{e} t\right)-\left(\frac{\partial \hat{C}_{+}^{(l)}}{\partial T}-\mathrm{i} \tilde{f} \hat{C}_{+}^{(l)}\right) \exp \left(\mathrm{i} \theta-\mathrm{i} \omega_{e} t\right) \boldsymbol{P}_{+} \\
& -\left(\frac{\partial \hat{C}_{-}^{(l)}}{\partial T}+\mathrm{i} \tilde{f} \hat{C}_{-}^{(l)}\right) \exp \left(-\mathrm{i} \theta+\mathrm{i} \omega_{e}^{*} t\right) \boldsymbol{P}_{-},
\end{aligned}
$$

where $\boldsymbol{P}_{ \pm}=\left(\hat{u}_{r 0 \pm}, \hat{u}_{\theta 0 \pm}, \hat{u}_{z 0 \pm}, \hat{\rho}_{0 \pm}\right)$ and the terms $\boldsymbol{S}_{ \pm}$and $\boldsymbol{S}_{ \pm 3}$ come from the azimuthal dependence $\exp ( \pm \mathrm{i} 2 \theta)$ of the straining flow. Note that the superscript of $\omega_{e}^{(l)}$ has been dropped for brevity. None of the terms $S_{ \pm} \exp ( \pm \mathrm{i} \theta)$ are exactly resonants. However, since the frequency $\omega_{e}$ is assumed to be of the same order as the strain, we can write $\omega_{e}^{*} t=-\omega_{e} t+\left(\omega_{e}^{*}+\omega_{e}\right) t=-\omega_{e} t+O(T)$ and the $O(T)$ term can be absorbed in the amplitudes $\hat{C}_{ \pm}^{(l)}$ by a rescaling. This means that the terms $S_{ \pm} \exp ( \pm \mathrm{i} \theta)$ can be considered to be resonant at order $\epsilon$ in addition to the terms $\overline{\boldsymbol{P}}_{ \pm} \exp ( \pm \mathrm{i} \theta)$ in (E 8). In order to remove the secular growth associated with these resonant terms, a compatibility condition needs to be imposed. In the related problem of the elliptic instability, Moore \& Saffman (1975) and Sipp (2000) use a scalar product involving all the velocity components (and also the pressure for Moore \& Saffman 1975) in order to apply the Fredholm alternative. However, such scalar product cannot be employed in the present case because the boundary terms that appear when computing the adjoint operator do not vanish at $r=\infty$ when $k \rightarrow 0$. This is because the eigensolution of the slow bending wave decays only algebraically and not exponentially with the radius when $k=0$. This difficulty can be circumvented by using a slightly different approach. We first write the first-order solution in the form

$\left[\tilde{\boldsymbol{u}}_{1}, \tilde{\rho}_{1}\right](r, \theta, t)=\left[\hat{\boldsymbol{u}}_{1+}, \hat{\rho}_{1+}\right](r) \exp \left(-\mathrm{i} \omega_{e} t+\mathrm{i} \theta\right)+\left[\hat{\boldsymbol{u}}_{1-}, \hat{\rho}_{1-}\right](r) \exp \left(\mathrm{i} \omega_{e}^{*} t-\mathrm{i} \theta\right)+[\ldots] \mathrm{e}^{ \pm 3 \mathrm{i} \theta}$.

Equations (E 3)-(E 7) are then reduced to a single equation for $\varphi_{1 \pm}=r \hat{u}_{r 1 \pm}$ :

$$
\mathscr{L}_{ \pm 1}\left(\varphi_{1 \pm}\right)=\hat{C}_{\mp}^{(l)} \exp \left( \pm \mathrm{i} \frac{\omega_{e}+\omega_{e}^{*}}{\epsilon} T\right) \mathscr{M}_{ \pm 1}\left(S_{ \pm}\right)-\left(\frac{\partial \hat{C}_{ \pm}^{(l)}}{\partial T} \mp \mathrm{i} \tilde{f} \hat{C}_{ \pm}^{(l)}\right) \mathscr{M}_{ \pm 1}\left(\boldsymbol{P}_{ \pm}\right)
$$


where the operator $\mathscr{L}_{m}$ corresponds to (4.11) and

$$
\begin{array}{r}
\mathscr{M}_{1}(S)=\frac{\mathrm{i} S^{(1)}}{s}+\frac{2 \Omega+R o^{(l)}-1}{s^{2}} S^{(2)}-\left(\frac{\zeta+R o^{(l)}-1}{Q r s}-\frac{Q^{\prime}}{Q^{2}}\right)\left[\frac{S^{(2)}}{r s}-k \frac{\mathrm{i} S^{(4)}+s S^{(3)}}{1-F_{h}^{(l)^{2}} s^{2}}\right] \\
-\frac{1}{Q}\left[\frac{S^{(2)}}{r s}-k \frac{\mathrm{i} S^{(4)}+s S^{(3)}}{1-F_{h}^{(l)^{2}} s^{2}}\right]^{\prime}, \quad(\mathrm{E} 11)
\end{array}
$$

with $s=-\omega_{e}+\Omega$ and where the prime denotes differentiation with respect to $r$. Because of the identity $S_{-}^{*}=\left(S_{+}^{(1)}, S_{+}^{(2)},-S_{+}^{(3)},-S_{+}^{(4)}\right)$, we have $\mathscr{M}_{-1}\left(S_{-}\right)=\left(\mathscr{M}_{1}\left(S_{+}\right)\right)^{*}$. Similarly, we have $\mathscr{M}_{-1}\left(\boldsymbol{P}_{-}\right)=\left(\mathscr{M}_{1}\left(\boldsymbol{P}_{+}\right)\right)^{*}$. Then, the Fredholm alternative can be applied to (E 10). However, some caution is needed because the operator $\mathscr{L}_{m}$ is singular at the radius $r_{c}$ where $s=0$. This critical radius is avoided in the complex plane by deforming the path of integration with the rule (3.38). However, this device cannot be used to evaluate the right-hand side of (E 10) since it involves not only $\left[\hat{\boldsymbol{u}}_{0+}, \hat{\rho}_{0+}\right]$ but also its complex conjugate, which is not valid in the same region of the complex plane. Accordingly, it is not possible to deform the path of integration and the structure of the viscous critical layer must be fully determined.

However, when $\omega_{e} \ll 1$, the critical radius is $r_{c} \sim \sqrt{m / \omega_{e}}$. Therefore, when $\omega_{e}$ is sufficiently small, $r_{c}$ is located outside the vortex core in the region where the basic vorticity $\zeta$ is zero or negligible. In the particular case of the Lamb-Oseen vortex, $\zeta$ decreases exponentially with $r$ and therefore the singular term of the operator $\mathscr{L}_{m}$, which is $-m \varphi \zeta^{\prime} /(r s)$, is exponentially small at the critical radius. For this reason, the singularity is negligible when $\omega_{e}$ is sufficiently small. In practice, the condition $\zeta^{\prime}\left(r_{c}\right) \approx 0$ is fulfilled when $\omega_{e} \lesssim 0.1$ for the Lamb-Oseen vortex. This explains why this singularity does not appear in the asymptotic analysis in $\S 3$ since the frequency is zero at leading order. In contrast, when $\omega_{e}$ increases beyond this limit, the operator $\mathscr{L}_{m}$ becomes truly singular at $r_{c}$. Similarly, the operator $\mathscr{M}_{1}$ is singular at $r_{c}$ but, when $\omega_{e}$ is small, the singularity occurs for large $r$ in a region where the values of $\mathscr{M}_{1}$ at $r_{c}$ are negligible compared to those in the vortex core. For these reasons, we can apply the Fredholm alternative directly to (E 10) when $\omega_{e} \lesssim 0.1$ for the Lamb-Oseen vortex. We stress that this condition will be fulfilled for the cases where the present approach will be used in Part 2. To do so, we introduce the scalar product defined by

$$
\langle\chi \mid \varphi\rangle=\int_{0}^{\infty} \chi \varphi \mathrm{d} r .
$$

Taking the scalar product of (E 10) multiplied by $r \hat{u}_{r 0 \pm}$ then leads to the following compatibility conditions after two integration by parts:

$$
\frac{\partial \hat{C}_{ \pm}^{(l)}}{\partial T}= \pm \mathrm{i} \tilde{f} \hat{C}_{ \pm}^{(l)}+\lambda_{ \pm} \hat{C}_{\bar{\mp}}^{(l)} \exp \left( \pm \mathrm{i} \frac{\omega_{e}+\omega_{e}^{*}}{\epsilon} T\right),
$$

where

$$
\lambda_{ \pm}=\frac{\left\langle r \hat{u}_{r 0 \pm} \mid \mathscr{M}_{ \pm 1}\left(\boldsymbol{S}_{ \pm}\right)\right\rangle}{\left\langle r \hat{u}_{r 0 \pm} \mid \mathscr{M}_{ \pm 1}\left(\boldsymbol{P}_{ \pm}\right)\right\rangle} .
$$

The identities mentioned above imply that $\lambda_{+}=\lambda_{-}^{*} \equiv \lambda$. The boundary terms appearing in the integration by parts always vanish for $r \rightarrow \infty$ even when $k \rightarrow 0$. We also emphasize that the integrands involved in the scalar products become negligible as $r$ increases well before the critical radius when $\omega_{e} \ll 1$. 
If we rescale the amplitudes as

$$
\hat{C}_{+}^{(l)}=C_{+}^{(l)} \mathrm{e}^{\mathrm{i} \omega_{e} T / \epsilon}, \quad \hat{C}_{-}^{(l)}=C_{-}^{(l)} \mathrm{e}^{-\mathrm{i} \omega_{e}^{*} T / \epsilon},
$$

(E 13) become

$$
\begin{aligned}
& \frac{\partial C_{+}^{(l)}}{\partial T}=-\mathrm{i}\left(\frac{\omega_{e}}{\epsilon}-\tilde{f}\right) C_{+}^{(l)}+\lambda C_{-}^{(l)}, \\
& \frac{\partial C_{-}^{(l)}}{\partial T}=\mathrm{i}\left(\frac{\omega_{e}^{*}}{\epsilon}-\tilde{f}\right) C_{-}^{(l)}+\lambda^{*} C_{+}^{(l)} .
\end{aligned}
$$

We now turn our attention to the mutual-induction effects. If we consider the perturbations due to a companion vortex, the additional forcing term $\boldsymbol{R}$ on the right-hand side of (E 3)-(E 7) is

$$
\boldsymbol{R}=-\frac{1}{\epsilon}\left(\left[\Omega \frac{\partial \tilde{u}_{r 0}^{(r)}}{\partial \theta}-2 \Omega \tilde{u}_{\theta 0}^{(r)}\right],\left[\Omega \frac{\partial \tilde{u}_{\theta 0}^{(r)}}{\partial \theta}+\zeta \tilde{u}_{r 0}^{(r)}\right], F_{h}^{(l)}{ }^{2} \Omega \frac{\partial \tilde{u}_{z 0}^{(r)}}{\partial \theta}, \Omega \frac{\partial \tilde{\rho}_{0}^{(r)}}{\partial \theta}\right),
$$

where $\left(\tilde{u}_{r 0}^{(r)}, u_{\theta 0}^{(r)}\right)$ is the horizontal velocity of the leading-order perturbation of the companion vortex expressed in the cylindrical coordinates $(r, \theta)$. This velocity field can be expressed far from the vortex centre by a streamfunction $\tilde{\boldsymbol{u}}_{0}^{(r)}=-\nabla \times \tilde{\psi}_{0}^{(r)} \boldsymbol{e}_{z}$, where

$$
\tilde{\psi}_{0}^{(r)}(\xi, \eta, t)=\tilde{\Gamma} C_{+}^{(r)}(T) \hat{\psi}_{0}(\xi) \mathrm{e}^{\mathrm{i} \eta}+\tilde{\Gamma} C_{-}^{(r)}(T) \hat{\psi}_{0}^{*}(\xi) \mathrm{e}^{-\mathrm{i} \eta},
$$

where $(\xi, \eta)$ are the cylindrical coordinates centred on the vortex $(r)$ (figure 1$)$. Note that the slow time dependences $\exp \left(-\mathrm{i} \omega_{e}^{(r)} t\right)$ and $\exp \left(\mathrm{i} \omega_{e}^{(r) *} t\right)$ have been directly included in the amplitudes $C_{+}^{(r)}(T)$ and $C_{-}^{(r)}(T)$, respectively. For $(\xi \approx \tilde{b}, \eta \approx \pi)$, this streamfunction is at leading orders in $r / \tilde{b}$, up to a constant:

$$
\begin{aligned}
& \tilde{\psi}_{0}^{(r)}(\xi, \eta, t)=\tilde{\Gamma} C_{+}^{(r)}\left[\left(\hat{\psi}_{0}^{\prime}(\tilde{b})+\frac{r^{2}}{8} \hat{\zeta}_{0}^{\prime}(\tilde{b})\right) r \cos \theta+\mathrm{i}\left(\frac{\hat{\psi}_{0}(\tilde{b})}{\tilde{b}}+\frac{r^{2}}{8} \frac{\hat{\zeta}_{0}(\tilde{b})}{\tilde{b}}\right) r \sin \theta\right] \\
& +\tilde{\Gamma} C_{-}^{(r)}\left[\left(\hat{\psi}_{0}^{\prime *}(\tilde{b})+\frac{r^{2}}{8} \hat{\zeta}_{0}^{\prime *}(\tilde{b})\right) r \cos \theta-\mathrm{i}\left(\frac{\hat{\psi}_{0}^{*}(\tilde{b})}{\tilde{b}}+\frac{r^{2}}{8} \frac{\hat{\zeta}_{0}^{*}(\tilde{b})}{\tilde{b}}\right) r \sin \theta\right], \quad(\mathrm{E} 20)
\end{aligned}
$$

where $\hat{\zeta}_{0}=\mathrm{d}^{2} \hat{\psi}_{0} / \mathrm{d} \xi^{2}+(1 / r) \mathrm{d} \hat{\psi}_{0} / \mathrm{d} \xi-\hat{\psi}_{0} / \xi^{2}$ is the vertical vorticity. Note that higher azimuthal components, e.g. $\cos m \theta$ or $\sin m \theta$ with $m>1$, are also present in (E 20), but they do not need to be considered since they are not resonant.

Similarly, the density perturbation of the companion vortex can be approximated at leading order in $r / \tilde{b}$ near $(\xi \approx \tilde{b}, \eta \approx \pi)$ by

$$
\begin{aligned}
\tilde{\rho}_{0}^{(r)} & =\tilde{\Gamma} C_{+}^{(r)} \hat{\rho}_{0}(\xi) \mathrm{e}^{\mathrm{i} \eta}-\tilde{\Gamma} C_{-}^{(r)} \hat{\rho}_{0}^{*}(\xi) \mathrm{e}^{-\mathrm{i} \eta} \\
& \approx \tilde{\Gamma} C_{+}^{(r)} r\left(\hat{\rho}_{0}^{\prime}(\tilde{b}) \cos \theta+\mathrm{i} \frac{\hat{\rho}_{0}(\tilde{b})}{\tilde{b}} \sin \theta\right)-\tilde{\Gamma} C_{-}^{(r)} r\left(\hat{\rho}_{0}^{*^{\prime}}(\tilde{b}) \cos \theta-\mathrm{i} \frac{\hat{\rho}_{0}^{*}(\tilde{b})}{\tilde{b}} \sin \theta\right),
\end{aligned}
$$

up to a constant. In contrast, the vertical velocity $\tilde{u}_{z 0}^{(r)}$ for $\xi \approx \tilde{b}$ scales like $\epsilon \tilde{\rho}_{0}^{(r)}$ and is thus one order in $\epsilon$ smaller and can be neglected here. In addition, since the potential vorticity of the perturbation is zero, far from the vortex core, we have the relationship $\hat{\zeta}_{0}(\tilde{b})=\mathrm{i} k \hat{\rho}_{0}(\tilde{b}) / R o^{(r)}$. At leading order in $\epsilon$, the perturbation can also be assumed to be in quasi-geostrophic balance so that $\hat{\rho}_{0}(\tilde{b})=-\mathrm{i} k \hat{\psi}_{0}(\tilde{b}) / R o^{(r)}$. 
With these mutual-induction effects, the compatibility conditions (E 16)-(E 17) become

$$
\begin{aligned}
\frac{\partial C_{+}^{(l)}}{\partial T} & =-\mathrm{i}\left(\frac{\omega_{e}}{\epsilon}-\tilde{f}\right) C_{+}^{(l)}+\lambda C_{-}^{(l)}+\frac{\mathrm{i} \kappa}{2}\left[C_{+}^{(r)}(\bar{\Psi}-\bar{\chi})-C_{-}^{(r)}\left(\bar{\Psi}^{*}+\bar{\chi}^{*}\right)\right], \\
\frac{\partial C_{-}^{(l)}}{\partial T} & =\mathrm{i}\left(\frac{\omega_{e}^{*}}{\epsilon}-\tilde{f}\right) C_{-}^{(l)}+\lambda^{*} C_{+}^{(l)}+\frac{\mathrm{i} \kappa^{*}}{2}\left[C_{+}^{(r)}(\bar{\Psi}+\bar{\chi})-C_{-}^{(r)}\left(\bar{\Psi}^{*}-\bar{\chi}^{*}\right)\right],
\end{aligned}
$$

where $\bar{\Psi}=\hat{\psi}_{0}(\tilde{b}) \tilde{b}, \bar{\chi}=-\hat{\psi}_{0}^{\prime}(\tilde{b}) \tilde{b}^{2}$ and

$$
\kappa=\frac{\left\langle r \hat{u}_{r 0+} \mid \mathscr{M}_{1}\left(\boldsymbol{U}_{+}\right)\right\rangle}{\left\langle r \hat{u}_{r 0+} \mid \mathscr{M}_{1}\left(\boldsymbol{P}_{+}\right)\right\rangle},
$$

with

$$
\boldsymbol{U}_{+}=\left(-\mathrm{i} \Omega-\mathrm{i} \frac{5 r^{2} \Omega k^{2}}{8 R o^{(r)^{2}}}, r \Omega^{\prime}+\Omega-\frac{k^{2} r^{2}\left(\Omega-r \Omega^{\prime}\right)}{8 R o^{(r)^{2}}}, 0, \mathrm{i} \frac{k \Omega r}{R o^{(r)}}\right) .
$$

When $k \rightarrow 0$, we obtain $\lambda=\mathrm{i}$ and $\kappa=1$, and we have $\bar{\Psi}=\Psi$ and $\bar{\chi}=\chi$ so that (E 21)-(E 22) recover (3.80)-(3.81). The dimensional equations for the displacement quantities which replace $(3.84)-(3.85)$ are

$$
\begin{aligned}
\frac{\partial \Delta x^{(l)}}{\partial \hat{t}}=- & \frac{\Gamma^{(r)}}{2 \pi b^{2}} \lambda_{i} \Delta y^{(l)}+\frac{\Gamma^{(r)}}{2 \pi b^{2}}\left(\kappa_{r} \bar{\Psi}_{r}+\kappa_{i} \bar{\chi}_{i}\right) \Delta y^{(r)}+\frac{\Gamma^{(r)}}{2 \pi b^{2}}\left(\kappa_{i} \bar{\chi}_{r}-\kappa_{r} \bar{\Psi}_{i}\right) \Delta x^{(r)} \\
& +\left(f-\frac{\Gamma^{(l)}}{2 \pi R^{(l)^{2}}} \omega_{e r}^{(l)}\right) \Delta y^{(l)}+\left(\frac{\left|\Gamma^{(l)}\right|}{2 \pi R^{(l)^{2}}} \omega_{e i}^{(l)}+\lambda_{r} \frac{\Gamma^{(r)}}{2 \pi b^{2}}\right) \Delta x^{(l)}, \quad(\mathrm{E} 25) \\
\frac{\partial \Delta y^{(l)}}{\partial \hat{t}}=- & \frac{\Gamma^{(r)}}{2 \pi b^{2}} \lambda_{i} \Delta x^{(l)}+\frac{\Gamma^{(r)}}{2 \pi b^{2}}\left(\kappa_{r} \bar{\chi}_{r}+\kappa_{i} \bar{\Psi}_{i}\right) \Delta x^{(r)}+\frac{\Gamma^{(r)}}{2 \pi b^{2}}\left(\kappa_{r} \bar{\chi}_{i}-\kappa_{i} \bar{\Psi}_{r}\right) \Delta y^{(r)} \\
& -\left(f-\frac{\Gamma^{(l)}}{2 \pi R^{(l)^{2}}} \omega_{e r}^{(l)}\right) \Delta x^{(l)}+\left(\frac{\left|\Gamma^{(l)}\right|}{2 \pi R^{(l)}{ }^{2}} \omega_{e i}^{(l)}-\lambda_{r} \frac{\Gamma^{(r)}}{2 \pi b^{2}}\right) \Delta y^{(l)} . \quad(\mathrm{E} 26)
\end{aligned}
$$

These improved equations will be shown in Part 2 to give accurate results for corotating vortex pairs when the Rossby number has $O(1)$ negative values.

\section{REFERENCES}

Abramowitz, M. \& Stegun, I. A. 1965 Handbook of Mathematical Functions. Dover.

AntKowiak, A. \& Brancher, P. 2007 On vortex rings around vortices: an optimal mechanism. J. Fluid Mech. 578, 295-304.

Batchelor, G. K. 1967 An Introduction to Fluid Dynamics. Cambridge University Press.

Billant, P. \& Chomaz, J.-M. $2000 a$ Experimental evidence for a new instability of a vertical columnar vortex pair in a strongly stratified fluid. J. Fluid Mech. 418, 167-188.

Billant, P. \& Chomaz, J.-M. $2000 b$ Theoretical analysis of the zigzag instability of a vertical columnar vortex pair in a strongly stratified fluid. J. Fluid Mech. 419, 29-63.

Billant, P. \& Chomaz, J.-M. 2001 Self-similarity of strongly stratified inviscid flows. Phys. Fluids 13, 1645-1651.

Billant, P., Deloncle, A., Chomaz, J. M. \& Otheguy, P. 2010 Zigzag instability of vortex pairs in stratified and rotating fluid. Part 2. Analytical and numerical analyses. J. Fluid Mech. doi:10.1017/S002211201000282X.

Billant, P., Dritschel, D. G. \& Chomaz, J.-M. 2006 Bending and twisting instabilities of columnar elliptical vortices in a rotating strongly stratified fluid. J. Fluid Mech. 561, 73-102.

Boulanger, N., Meunier, P. \& Le Dizès, S. 2007 Structure of a stratified tilted vortex. J. Fluid Mech. 583, 443-458.

Bristol, R. L., Ortega, J. M., Marcus, P. S. \& Savas, O. 2004 On cooperative instabilities of parallel vortex pairs. J. Fluid Mech. 517, 331-358. 
Crow, S. C. 1970 Stability theory for a pair of trailing vortices. AIAA J. 8, 2172-2179.

Deloncle, A., Billant, P. \& Chomaz, J.-M. 2008 Nonlinear evolution of the zigzag instability in stratified fluids: a shortcut on the route to dissipation. J. Fluid Mech. 599, 229-239.

Drazin, P. G. \& ReID, W. H. 1981 Hydrodynamic Stability. Cambridge University Press.

Dritschel, D. G. \& DE LA Torre JuÁrez, M. 1996 The instability and breakdown of tall columnar vortices in a quasi-geostrophic fluid. J. Fluid Mech. 328, 129-160.

Dritschel, D. G., de la Torre Juárez, M. \& Ambaum, M. H. P. 1999 The three-dimensional vortical nature of atmospheric and oceanic turbulent flows. Phys. Fluids 11 (6), 1512-1520.

Gil, A., Segura, J. \& Temme, N. M. 2002 Algorithm 822: GIZ, HIZ: two Fortran 77 routines for the computation of complex Scorer functions. ACM Trans. Math. Softw. 28 (4), 436-447.

Huerre, P. \& Rossi, M. 1998 Hydrodynamic instabilities in open flows. In Hydrodynamics and Nonlinear Instabilities (ed. C. Godreche \& P. Manneville), pp. 81-294. Cambridge University Press.

JimeneZ, J. 1975 Stability of a pair of co-rotating vortices. Phys. Fluids 18, 1580-1582.

LE Dizès, S. 2004 Viscous critical-layer analysis of vortex normal modes. Stud. Appl. Math. 112 (4), 315-332.

LE Dizès, S. 2008 Inviscid waves on a Lamb-Oseen vortex in a rotating stratified fluid: consequences on the elliptic instability. J. Fluid Mech. 597, 283-303.

LE Dizès, S. \& LAPORTE, F. 2002 Theoretical predictions for the elliptical instability in a two-vortex flow. J. Fluid Mech. 471, 169-201.

Leibovich, S., Brown, S. N. \& Patel, Y. 1986 Bending waves on inviscid columnar vortices. J. Fluid Mech. 173, 595-624.

Lin, C. C. 1955 The Theory of Hydrodynamics Stability. Cambridge University Press.

Mason, D. M. \& Kerswell, R. R. 1999 Nonlinear evolution of the elliptical instability: an example of inertial wave breakdown. J. Fluid Mech. 396, 73-108.

Meunier, P. \& LeWeke, T. 2005 Elliptic instability of a co-rotating vortex pair. J. Fluid Mech. 533, $125-159$.

Michalke, A. \& Timme, A. 1967 On the inviscid instability of certain two-dimensional vortex-type flows. J. Fluid Mech. 29 (4), 647-666.

MiYazaki, T., Shimada, M. \& Takahashi, N. 2000 Quasigeostrophic wire-vortex model. J. Phys. Soc. Jpn. 69 (10), 3233-3243.

Moore, D. W. \& Saffman, P. G. 1972 Motion of a vortex filament with axial-flow. Phil. Trans. R. Soc. Lond. A 272 (1226), 403-429.

Moore, D. W. \& SAFFman, P. G. 1975 The instability of a straight vortex filament in a strain field. Proc. R. Soc. Lond. 346, 413-425.

Otheguy, P., Billant, P. \& Chomaz, J. M. $2006 a$ The effect of planetary rotation on the zigzag instability of co-rotating vortices in a stratified fluid. J. Fluid Mech. 553, 273-281.

Otheguy, P., Billant, P. \& Chomaz, J. M. 2007 Theoretical analysis of the zigzag instability of a vertical co-rotating vortex pair in a strongly stratified fluid. J. Fluid Mech. 584, 103-123.

Otheguy, P., Chomaz, J. M. \& Billant, P. $2006 b$ Elliptic and zigzag instabilities on co-rotating vertical vortices in a stratified fluid. J. Fluid Mech. 553, 253-272.

Riley, J. J. \& LeLONG, M.-P. 2000 Fluid motions in the presence of strong stable stratification. Annu. Rev. Fluid Mech. 32, 617-657.

Rossi, M. 2000 Of vortices and vortical layers: an overview. In Vortex Structure and Dynamics (ed. A. Maurel \& P. Petitjeans), Lecture Notes in Physics, vol. 555, pp. 40-123. Springer.

Saffman, P. G. 1992 Vortex Dynamics. Cambridge University Press.

SIPP, D. 2000 Weakly nonlinear saturation of short-wave instabilities in a strained Lamb-Oseen vortex. Phys. Fluids 12 (7), 1715-1729.

SipP, D. \& JACQUin, L. 2003 Widnall instabilities in vortex pairs. Phys. Fluids 15, 1861-1874.

Waite, M. L. \& Smolarkiewicz, P. K. 2008 Instability and breakdown of a vertical vortex pair in a strongly stratified fluid. J. Fluid Mech. 606, 239-273.

WalefFe, F. 1990 On the 3-dimensional instability of strained vortices. Phys. Fluids 2 (1), 76-80.

Widnall, S. E., Bliss, D. \& Zalay, A. 1971 Theo retical and experimental study of the stability of a vortex pair. In Aircraft Wake Turbulence (ed. A. Goldburg, J. H. Olsen \& M. Rogers), pp. 305-329. Plenum. 